\title{
Electrical Probes of the Non-Abelian Spin Liquid in Kitaev Materials
}

\author{
David Aasen, ${ }^{1,2}$ Roger S. K. Mong, ${ }^{3,4}$ Benjamin M. Hunt,,${ }^{5,4}$ David Mandrus $\odot,{ }^{6,7}$ and Jason Alicea $\odot^{8,9}$ \\ ${ }^{1}$ Microsoft Quantum, Microsoft Station Q, University of California, \\ Santa Barbara, California 93106-6105 USA \\ ${ }^{2}$ Kavli Institute for Theoretical Physics, University of California, Santa Barbara, California 93106, USA \\ ${ }^{3}$ Department of Physics and Astronomy, University of Pittsburgh, Pittsburgh, Pennsylvania 15260, USA \\ ${ }^{4}$ Pittsburgh Quantum Institute, Pittsburgh, Pennsylvania 15260, USA \\ ${ }^{5}$ Department of Physics, Carnegie Mellon University, Pittsburgh, Pennsylvania 15213, USA \\ ${ }^{6}$ Department of Materials Science and Engineering, University of Tennessee, \\ Knoxville, Tennessee 37996, USA \\ ${ }^{7}$ Materials Science and Technology Division, Oak Ridge National Laboratory, \\ Oak Ridge, Tennessee 37831, USA \\ ${ }^{8}$ Department of Physics and Institute for Quantum Information and Matter, \\ California Institute of Technology, Pasadena, California 91125, USA \\ ${ }^{9}$ Walter Burke Institute for Theoretical Physics, California Institute of Technology, \\ Pasadena, California 91125, USA
}

(Received 5 March 2020; revised 12 May 2020; accepted 19 May 2020; published 17 July 2020)

\begin{abstract}
Recent thermal-conductivity measurements evidence a magnetic-field-induced non-Abelian spin-liquid phase in the Kitaev material $\alpha-\mathrm{RuCl}_{3}$. Although the platform is a good Mott insulator, we propose experiments that electrically probe the spin liquid's hallmark chiral Majorana edge state and bulk anyons, including their exotic exchange statistics. We specifically introduce circuits that exploit interfaces between electrically active systems and Kitaev materials to "perfectly" convert electrons from the former into emergent fermions in the latter-thereby enabling variations of transport probes invented for topological superconductors and fractional quantum-Hall states. Along the way, we resolve puzzles in the literature concerning interacting Majorana fermions, and also develop an anyon-interferometry framework that incorporates nontrivial energy-partitioning effects. Our results illuminate a partial pathway toward topological quantum computation with Kitaev materials.
\end{abstract}

DOI: 10.1103/PhysRevX.10.031014

Subject Areas: Condensed Matter Physics

\section{INTRODUCTION}

The field of topological quantum computation pursues phases of matter supporting emergent particles known as "non-Abelian anyons" to ultimately realize scalable, intrinsically fault-tolerant qubits $[1,2]$. This technological promise derives from three deeply linked non-Abelian-anyon features: First, nucleating well-separated non-Abelian anyons generates a ground-state degeneracy consisting of states that cannot be distinguished from one another by local measurements. Qubits encoded in this subspace enjoy built-in protection from environmental noise by virtue of local indistinguishability. Second, they obey non-Abelian braiding statistics. That is, adiabatically exchanging pairs of nonAbelian anyons effects "rigid" noncommutative rotations

Published by the American Physical Society under the terms of the Creative Commons Attribution 4.0 International license. Further distribution of this work must maintain attribution to the author(s) and the published article's title, journal citation, and DOI. within the ground-state manifold-thus producing faulttolerant qubit gates. And third, pairs of non-Abelian anyons brought together in space can "fuse" to at least two different types of particles; detecting the fusion outcome provides a means of qubit readout.

Fulfilling this potential requires, at an absolute minimum, synthesizing a non-Abelian host material and developing practical means of controlling and probing the constituent anyons. The observed fractional quantum-Hall phase at filling factor $\nu=5 / 2$ [3], widely expected to realize the non-Abelian Moore-Read state or cousins thereof [4-8], provided the first candidate topological-quantum-computing medium. Non-Abelian anyons in this setting carry electric charge (e.g., $e / 4)$, and hence, can be manipulated via gating and probed using ingenious electrical interferometry schemes [9-11]. While experimental efforts in this direction continue [12], during the past decade intense experimental activity has focused on "engineered" two-dimensional (2D) and especially one-dimensional (1D) topological superconductors $[13,14]$ as alternative platforms. These exotic superconductors can be assembled from heterostructures 
(a)

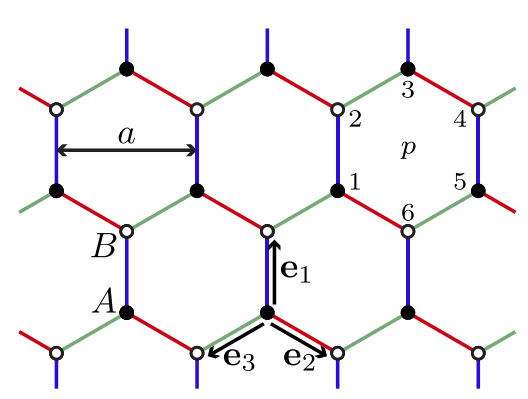

(b)

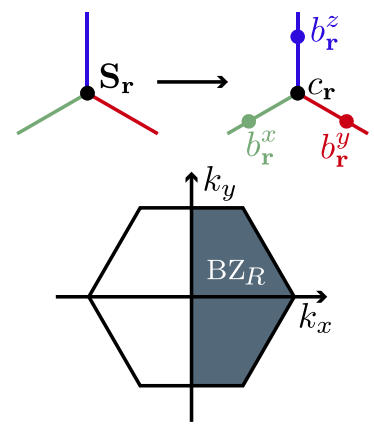

(c)

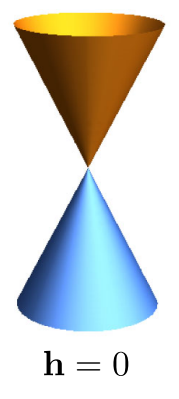

(d)

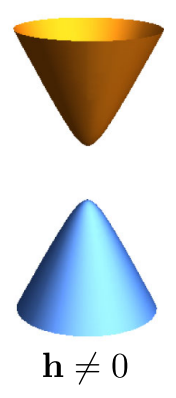

FIG. 1. (a) Lattice structure for the Kitaev honeycomb model. Spins exhibit bond-dependent nearest-neighbor interactions, with the $x$, $y$, and $z$ components, respectively, coupling along green, red, and blue bonds. Vectors $\mathbf{e}_{1,2,3}$ point from the $A$ sublattice (solid circles) to the $B$ sublattice (open circles). (b) Sketch of the Majorana-fermion representation of the spin operators [Eq. (4)] used for the exact solution. The $b_{\mathbf{r}}^{x, y, z}$ operators combine to form a $\mathbb{Z}_{2}$ gauge field, while the $c_{\mathbf{r}}$ operators define itinerant Majorana fermions that hop between nearest-neighbor sites. Hermiticity of the $c_{\mathbf{r}}$ 's allows the kinetic energy to be expressed as a sum over momenta in the right half of the Brilllouin zone (BZ $\mathrm{BZ}_{R}$, shaded region). (c) In the gapless spin-liquid phase, the fermionic spectrum features a single massless Dirac cone. (d) Breaking time-reversal symmetry via an applied magnetic field opens a gap at the Dirac point, generating a non-Abelian spinliquid phase.

involving ordinary, weakly correlated materials yet share similar non-Abelian properties to the Moore-Read state (for reviews, see Refs. [15-25]). Specifically, the charged nonAbelian excitations in the Moore-Read state are replaced by non-Abelian defects-i.e., domain walls and superconducting vortices - that bind Majorana zero modes. In a topological superconductor, Majorana zero modes are equal superpositions of electrons and holes and thus carry no net charge. They do carry a physical-fermion-parity degree of freedom, however, and are thus amenable to electronic probes including tunneling spectroscopy, interferometry, Josephson measurements, etc.; see, e.g., Refs. [14,26-30]. In fact, detailed blueprints exist for scalable topologicalquantum-computation hardware based on 1D-topologicalsuperconductor arrays, relying largely on electrical tools for operation [31].

Still more recently, experiments suggest the emergence of yet another variant of the Moore-Read state, but in a fundamentally different physical setting from those above: the honeycomb "Kitaev material" $\alpha-\mathrm{RuCl}_{3}$ [32,33]. As background, consider a honeycomb lattice of spin-1/2 moments governed by a Hamiltonian of the form

$$
H=-\sum_{\left\langle\mathbf{r r}^{\prime}\right\rangle} K S_{\mathbf{r}}^{\gamma} S_{\mathbf{r}^{\prime}}^{\gamma}-\sum_{\mathbf{r}} \mathbf{h} \cdot \mathbf{S}_{\mathbf{r}}+\cdots
$$

The first term encodes bond-dependent spin interactions, with $\gamma=x$ on the green bonds of Fig. 1(a), $\gamma=y$ on red bonds, and $\gamma=z$ on blue bonds; note the strong frustration arising from these competing spin couplings, which suppresses the tendency for conventional symmetry-breaking order. The second term in the Hamiltonian accounts for the possible presence of a Zeeman field $\mathbf{h}$, while the ellipsis denotes additional allowed perturbations.
When only the $K$ term is present, the Hamiltonian reduces to Kitaev's famed exactly solvable honeycomb model [34]. Here the ground state realizes a time-reversal-invariant quantum spin liquid with gapless, emergent Majorana fermions coupled to a $\mathbb{Z}_{2}$ gauge field. For this paper, it is crucial to distinguish emergent Majorana fermions from physical Majorana fermions that appear as excitations at the boundaries of two- and three-dimensional topological superconductors. The latter Majorana fermions are built from ordinary electronic degrees of freedom, whereas the former represent bona fide fractionalized quasiparticles born within a purely bosonic spin system. It follows that physical Majorana fermions can shuttle between the host topological superconductor and a conventional electronic medium (e.g., a lead); conversely, emergent Majorana fermions live exclusively in the spin liquid.

Breaking time-reversal symmetry generates even more striking physics: A nonzero Zeeman field $\mathbf{h}$ gaps out the Majorana fermions, yielding a non-Abelian spin liquid exhibiting a fully gapped bulk and a gapless, chiral Majorana fermion edge state that underlies quantized thermal Hall conductance [34]. This phase supports two nontrivial quasiparticle types: massive emergent Majorana fermions and "Ising" non-Abelian anyons. The latter can be viewed as electrically neutral counterparts of the nonAbelian anyons in the Moore-Read state. Alternatively, they comprise deconfined cousins of non-Abelian defects in topological superconductors that bind Majorana zero modes carrying an emergent rather than physical-fermion-parity degree of freedom.

Jackeli and Khaliullin established that a class of strongly spin-orbit-coupled Mott insulators can, quite remarkably, be well modeled by Eq. (1) with inevitably present corrections represented by the ellipsis being "small" [35]. 
Their pioneering result opened up the now experimentally active field of Kitaev materials whose spins interact predominantly via bond-dependent spin interactions of the type built into Kitaev's honeycomb model [36-39]. All honeycomb-lattice Kitaev materials studied to date- $\alpha-\mathrm{RuCl}_{3}$ included [40] — magnetically order at zero field. Evidently, perturbations beyond the $K$ term in Eq. (1), while nominally small, destabilize the gapless quantum spin liquid [41] (various experiments nevertheless report residual fractionalization signatures at "high" energies [42-52]). In $\alpha-\mathrm{RuCl}_{3}$, applying an approximately $10 \mathrm{~T}$ in-plane magnetic field destroys the zero-field magnetic ordering [53]. Numerous experiments are consistent with the fascinating possibility that the system then enters the non-Abelian spin-liquid phase highlighted above [50,54-61]. Most strikingly, Kasahara et al. [60] report thermal-Hall-conductance measurements that agree well with the quantized value expected from the hallmark chiral Majorana edge mode. This experiment withstood some initial theoretical scrutiny [62,63], and has very recently been extended in Ref. [64].

Can one plausibly exploit $\alpha-\mathrm{RuCl}_{3}$ (or perhaps some related Kitaev materials) for topological quantum computation? This question is well motivated on at least two fronts. For one, the energy scales appear quite favorable. In Refs. [60,64], quantized thermal Hall conductance persists up to temperatures of roughly $5 \mathrm{~K}$, suggesting a spin-liquid bulk gap of similar magnitude - an encouraging figure compared to the gap expected in most other candidate nonAbelian platforms [65]. Moreover, $\alpha-\mathrm{RuCl}_{3}$ affords a great deal of materials-science flexibility [66-69]: It is exfoliatable, amenable to nanofabrication, can be readily interfaced with other materials, etc.

Manipulating and probing the anyons as required for advanced applications nevertheless poses a major outstanding challenge. In essence, the detailed road maps developed for quantum-Hall and topological superconductor platforms - which again heavily invoke electrical tools-need to be largely rewritten for non-Abelian spin liquids in Kitaev materials because they are electrically inert Mott insulators. Two subclasses of problems naturally arise here: (i) devising feasible techniques for creating, transporting, and fusing Ising anyons on demand in Kitaev materials and (ii) developing schemes for unambiguously detecting individual emergent fermions and Ising anyons as well as their nontrivial statistics. Vacancies and spin impurities appear to be promising ingredients for item (i). At least in the gapless spin-liquid phase of Kitaev's honeycomb model, both have been shown to trap $\mathbb{Z}_{2}$-flux excitations [70-72], which evolve into Ising anyons upon entering the non-Abelian phase. We leave detailed investigations of this issue for future work, and instead propose a series of experiments that directly tackle item (ii).

Our primary innovation is that, counterintuitively, lowvoltage electrical transport can be profitably employed to probe the detailed structure of non-Abelian spin liquids, their Mott-insulating character notwithstanding. We build off of seminal theory works that highlight the possibility of coherently converting physical fermions into emergent deconfined quasiparticles in Abelian spin liquids [73] and non-Abelian quantum-Hall phases [74] to probe fractionalization [75]. We pursue a complementary approach that closely relates to the physics of "fermion condensation" put on rigorous mathematical foundation in a similar setting in Ref. [76]. Specifically, we introduce a series of circuits that interface electronically active systems-notably proximitized $\nu=1$ integer quantum-Hall states, though other choices are possible — with Kitaev materials realizing a non-Abelian spin liquid. Strong interactions at their interface can effectively "sew up" these very different subsystems, leading to a striking and exceedingly useful phenomenon: A physical electron injected at low energies on the electronically active side converts with unit probability into an emergent fermion in the spin liquid.

Our circuits exploit this perfect conversion process to electrically reveal (via universal conductance signatures) the spin liquid's chiral Majorana edge state, bulk emergent fermions, and bulk Ising anyons, using variations of transport techniques developed for topological superconductors and fractional quantum-Hall states. Figures 8-10 sketch the corresponding setups. The electrical conductance of these circuits changes qualitatively upon perturbing the Kitaev material (again, an electrically inert element), e.g., to add or remove even a single bulk emergent fermion or Ising anyon; we argue that this feature makes our predictions especially unambiguous. Moreover, the circuits designed to detect individual bulk quasiparticles rely on interferometric signatures that further unambiguously reveal the non-Abelian statistics of Ising anyons as well as the nontrivial mutual statistics between Ising anyons and emergent fermions.

These results collectively establish a partial road map toward utilizing Kitaev materials for topological quantum computation. En route to putting our predictions on firm footing, we introduce some nontrivial technical innovations as well. First, we resolve an outstanding puzzle in the literature concerning interacting Majorana fermions. Specifically, the interaction strength required to induce an instability in a self-dual Majorana chain has been found to vary by orders of magnitude depending on subtle variations in the microscopic interaction (for a recent review, see Ref. [77]). We explain this peculiar behavior as arising from interaction-dependent renormalization of kinetic energy for the Majorana chain. Second, we analyze anyon interferometry in a new regime using a phenomenological picture combined with rigorous formalism that incorporates crucial energy-partitioning effects. The framework that we develop here could prove valuable in a variety of other contexts.

The remainder of the paper is organized as follows. We begin in Sec. II by reviewing the phenomenology of the Kitaev honeycomb model. Section III explores interacting 
helical Majorana fermions from several perspectives, and then Sec. IV bootstraps off of those results to describe how a $\nu=1$ quantum-Hall state can be sewn (in a precise sense) to a non-Abelian spin liquid with the aid of a superconductor. In the next three sections, we introduce circuits that use this "sewing" to electrically interrogate a nonAbelian spin liquid: Section $\mathrm{V}$ focuses on electrical detection of the chiral Majorana edge state, Sec. VI introduces a circuit that probes bulk Ising anyons, and Sec. VII introduces an interferometer that probes both bulk Ising anyons and emergent fermions, as well as nonAbelian statistics. We conclude and highlight numerous open questions in Sec. VIII. Several Appendixes provide additional details and supplementary results on our circuits as well as interacting Majorana-fermion models.

\section{KITAEV HONEYCOMB MODEL PHENOMENOLOGY}

To set the stage, this section reviews the phenomenology of the Kitaev honeycomb model [34], focusing in particular on universal properties of the non-Abelian spin-liquid phase. We also establish various conventions here that are employed throughout.

\section{A. Gapless spin liquid}

We start with the "pure" Kitaev honeycomb model at zero magnetic field:

$$
H_{K}=-\sum_{\left\langle\mathbf{r r}^{\prime}\right\rangle} K S_{\mathbf{r}}^{\gamma} S_{\mathbf{r}^{\prime}}^{\gamma}
$$

Once again, we have $\gamma=x, y$, and $z$ respectively on green, red, and blue bonds of Fig. 1(a). For any hexagonal plaquette $p, H_{K}$ commutes with the operator

$$
W_{p}=S_{1}^{x} S_{2}^{y} S_{3}^{z} S_{4}^{x} S_{5}^{y} S_{6}^{z}
$$

where sites $1,2, \ldots, 6$ around plaquette $p$ are labeled as in Fig. 1(a). The resulting extensive number of conserved quantities ultimately enables an exact solution. To this end, we reexpress the spins via

$$
S_{\mathbf{r}}^{\alpha}=\frac{i}{2} b_{\mathbf{r}}^{\alpha} c_{\mathbf{r}}
$$

on the right side, $b_{\mathbf{r}}^{\alpha}$ and $c_{\mathbf{r}}$ denote Majorana-fermion operators that are Hermitian, square to the identity, and anticommute with one another. For an illustration, see Fig. 1(b). Remaining faithful to the original spin- $1 / 2$ Hilbert space requires enforcing the local constraint $b_{\mathbf{r}}^{x} b_{\mathbf{r}}^{y} b_{\mathbf{r}}^{z} c_{\mathbf{r}}=+1$ at every site.

In the Majorana representation, the Hamiltonian becomes

$$
H_{K}=\frac{K}{4} \sum_{\left\langle\mathbf{r} \mathbf{r}^{\prime}\right\rangle} i \hat{u}_{\mathbf{r r}^{\prime}} c_{\mathbf{r}} c_{\mathbf{r}^{\prime}}
$$

Above, we introduce link variables $\hat{u}_{\mathbf{r r}^{\prime}} \equiv i b_{\mathbf{r}}^{\gamma} b_{\mathbf{r}^{\prime}}^{\gamma} \in \pm 1$ that, crucially, commute with each other and with the Hamiltonian. The link variables can thus be treated as classical parameters - thereby reducing the model to a freefermion problem in any fixed $\hat{u}_{\mathbf{r r}^{\prime}}$ configuration [78]. Physically, $\hat{u}_{\mathbf{r r}^{\prime}}$ is a $\mathbb{Z}_{2}$ gauge field whose flux around plaquette $p$ is proportional to the conserved $W_{p}$ operator in Eq. (3) (hence, the absence of nontrivial dynamics).

The ground state of Eq. (5) arises in the sector with $\mathbb{Z}_{2}$ gauge flux of $\pi$ through every hexagonal plaquette [79]. Let us decompose the honeycomb lattice into $A$ and $B$ sublattices, and also introduce vectors $\mathbf{e}_{j=1,2,3}$ that link the two sublattices; see Fig. 1(a). A convenient gauge encoding $\pi$ flux per plaquette is $\hat{u}_{\mathbf{r}, \mathbf{r}+\mathbf{e}_{j}}=+1$ for all $\mathbf{r}$ on sublattice $A$. Inserting this gauge choice into Eq. (5) yields a Hamiltonian

$$
\tilde{H}_{K}=\frac{K}{4} \sum_{\mathbf{r} \in A} \sum_{j=1}^{3} i c_{\mathbf{r}} c_{\mathbf{r}+\mathbf{e}_{j}}
$$

that describes the ground-state flux sector. One can view Eq. (6) as an analog of graphene wherein Majorana fermions hop between nearest-neighbor honeycomb sites. To obtain the spectrum of $H_{K}$, we pass to momentum space, employing conventions such that

$$
\begin{aligned}
c_{\mathbf{r} \in A / B} & =\sqrt{\frac{2}{N_{\text {u.c. }}} \sum_{\mathbf{k} \in \mathrm{BZ}} e^{i \mathbf{k} \cdot \mathbf{r}} c_{A / B \mathbf{k}}} \\
& =\sqrt{\frac{2}{N_{\text {u.c. }}}} \sum_{\mathbf{k} \in \mathrm{BZ}}\left(e^{i \mathbf{k} \cdot \mathbf{r}} c_{A / B \mathbf{k}}+e^{-i \mathbf{k} \cdot \mathbf{r}} c_{A / B \mathbf{k}}^{\dagger}\right),
\end{aligned}
$$

where $N_{\text {u.c. }}$ is the number of unit cells. The momentumspace operators so defined satisfy $\left\{c_{\alpha \mathbf{k}}, c_{\beta \mathbf{k}^{\prime}}^{\dagger}\right\}=\delta_{\alpha \beta} \delta_{\mathbf{k}, \mathbf{k}^{\prime}}$ and $c_{\alpha \mathbf{k}}=c_{\alpha-\mathbf{k}}^{\dagger}$ (reflecting Hermiticity of $c_{\mathbf{r}}$ ). In the second line of Eq. (7), we use the latter property to express $c_{\mathbf{r}}$ as a sum over momenta in the right half of the Brillouin zone $\left(\mathrm{BZ}_{R}\right)$, i.e., $\mathbf{k}$ with $k_{x}>0$ as shown in Fig. 1(b). Defining a two-component spinor $C_{\mathbf{k}}^{\dagger}=\left[c_{A \mathbf{k}}^{\dagger} c_{B \mathbf{k}}^{\dagger}\right]$ and a function $\xi(\mathbf{k})=-i(K / 2) \sum_{j} e^{-i \mathbf{k} \cdot \mathbf{e}_{j}}$, Eq. (6) becomes

$$
\tilde{H}_{K}=\sum_{\mathbf{k} \in \mathrm{BZ}_{R}} C_{\mathbf{k}}^{\dagger}\left[\begin{array}{cc}
0 & \xi^{*}(\mathbf{k}) \\
\xi(\mathbf{k}) & 0
\end{array}\right] C_{\mathbf{k}} .
$$

The resulting single-particle energies are $\pm|\xi(\mathbf{k})|^{2}$, and the many-particle ground state populates all negative energy levels.

This ground state realizes the gapless spin-liquid phase of Kitaev's honeycomb model. Specifically, Eq. (8) 
describes gapless (emergent) fermion excitations with a single massless Dirac cone centered at momentum $\mathbf{Q}=(4 \pi / 3 a) \hat{\mathbf{x}}$, with $a$ the lattice constant. See Fig. 1(c). We now focus on these gapless excitations by writing $\mathbf{k}=$ $\mathbf{Q}+\mathbf{q}$ and retaining only modes with "small" q. Equation (8) then reduces to the following effective Dirac Hamiltonian that captures low-energy fermionic excitations in the groundstate flux sector:

$$
\begin{aligned}
\mathcal{H}_{\text {eff }} & =v_{\text {bulk }} \int_{\mathbf{q}} \Psi_{\mathbf{q}}^{\dagger}\left(q_{x} \sigma^{y}-q_{y} \sigma^{x}\right) \Psi_{\mathbf{q}} \\
& =v_{\text {bulk }} \int_{\mathbf{r}} \Psi^{\dagger}\left(-i \partial_{x} \sigma^{y}+i \partial_{y} \sigma^{x}\right) \Psi .
\end{aligned}
$$

Here, $v_{\text {bulk }}=\sqrt{3} a K / 4, \Psi_{\mathbf{q}} \propto C_{\mathbf{Q}+\mathbf{q}}$, and in the last line we Fourier transform back to real space. Furthermore, we employ $\hbar=1$ units, and continue to do so throughout (for clarity, however, we express the conductance quantum as $e^{2} / h$ ). This gapless spin-liquid phase also admits gapped $\mathbb{Z}_{2}$-flux excitations that are not captured by $\mathcal{H}_{\text {eff }}$.

Suppose that we now supplement Eq. (2) with generic perturbations that preserve translation symmetry and timereversal symmetry $\mathcal{T}$, leading to a Hamiltonian of the form

$$
H=H_{K}+\cdots .
$$

Despite the loss of exact solvability, one can address the stability of the gapless spin liquid from the viewpoint of the effective low-energy theory. The original spin operators transform under $\mathcal{T}$ according to $\mathbf{S}_{\mathbf{r}} \rightarrow-\mathbf{S}_{\mathbf{r}}$. Within the ground-state flux sector, $\mathcal{T}$ sends $c_{\mathrm{r} \in A} \rightarrow c_{\mathrm{r} \in A}$ and $c_{\mathbf{r} \in B} \rightarrow-c_{\mathbf{r} \in B}$, and in turn transforms the low-energy Dirac field via $\Psi \rightarrow \sigma^{z}\left(\Psi^{\dagger}\right)^{t}$. The only translationally invariant perturbation to Eq. (9) that can open an energy gap is the mass term $m \Psi^{\dagger} \sigma^{z} \Psi$ - which is odd under $\mathcal{T}$ and cannot appear provided time-reversal symmetry persists. Consequently, the gapless spin liquid constitutes a stable symmetry-protected phase with some finite tolerance to the ellipsis in Eq. (10).

\section{B. Non-Abelian spin liquid}

In this paper, we are primarily interested in the physics resulting when time-reversal symmetry is explicitly broken by an applied magnetic field. The field modifies Eq. (10) to

$$
H=H_{K}-\sum_{\mathbf{r}} \mathbf{h} \cdot \mathbf{S}_{\mathbf{r}}+\cdots
$$

On symmetry grounds [80], the Zeeman term can be expanded in terms of low-energy degrees of freedom as $\mathbf{h} \cdot \mathbf{S}_{\mathbf{r}} \sim \beta \Psi^{\dagger} \sigma^{z} \Psi+\cdots$. Here, $\beta \propto|\mathbf{h}|$ is a nonuniversal constant that vanishes only for fine-tuned field orientations 64]], while the ellipsis denotes additional symmetryallowed terms that are unimportant for our purposes and are henceforth dropped. The effective low-energy Hamiltonian accordingly now reads

$$
\mathcal{H}_{\text {eff }}=\int_{\mathbf{r}} \Psi^{\dagger}\left[v_{\text {bulk }}\left(-i \partial_{x} \sigma^{y}+i \partial_{y} \sigma^{x}\right)+m \sigma^{z}\right] \Psi
$$

with $m \propto|\mathbf{h}|$, and describes emergent fermions with a gapped Dirac spectrum illustrated in Fig. 1(d). [Without the generic perturbations that we implicitly include in Eq. (11), the Dirac gap would scale like $h^{x} h^{y} h^{z}$ instead of $|\mathbf{h}|$. We stress that this fine-tuned behavior is a pathology of perturbating about the exactly solvable $H_{K}$ Hamiltonian as Ref. [80] discusses in detail.]

The resulting field-induced phase realizes a non-Abelian spin liquid with Ising topological order. Although the bulk is fully gapped, the system's boundary hosts a single emergent chiral Majorana mode with central charge $c=1 / 2$. (One can trace the edge state's existence to the quantized half-integer thermal Hall conductance that arises from gapping out a single Dirac cone; for related problems, see Refs. [81-84].) Low-energy edge excitations are described by the continuum Hamiltonian

$$
\mathcal{H}_{\text {edge }}=\int_{x}\left(-i v_{\text {edge }} \gamma \partial_{x} \gamma\right)
$$

where $v_{\text {edge }}$ is a nonuniversal velocity [85], $x$ is a coordinate along the boundary, and $\gamma(x)$ is a Majorana-fermion field. (For clarity, we employ subscripts that distinguish edge and bulk velocities, though later we abandon such a notation.) Here and below, we normalize continuum Majorana fields such that

$$
\left\{\gamma(x), \gamma\left(x^{\prime}\right)\right\}=\frac{1}{2} \delta\left(x-x^{\prime}\right) .
$$

With this choice, the energy for an edge excitation with momentum $k$ is simply $v k$. Note that Eq. (13) exhibits a global $\mathbb{Z}_{2}$ symmetry that sends $\gamma \rightarrow-\gamma$, which as we see in Sec. III A has important practical consequences for the interfaces that we exploit later in this paper.

The bulk of the non-Abelian spin liquid supports three gapped quasiparticle types. First, there are nonfractionalized bosonic excitations-as in any phase of matter-that we call trivial particles labeled by $\mathbb{1}$. Second, the system hosts more exotic gapped emergent fermions ( $\psi$ particles) captured by the effective Hamiltonian in Eq. (12). Third, and most interestingly, gapped $\mathbb{Z}_{2}$-flux excitations bind emergent Majorana zero modes and realize "Ising anyons" ( $\sigma$ particles) with non-Abelian braiding statistics. These quasiparticle types obey the following nontrivial "fusion rules,"

$\psi \otimes \psi \cong \mathbb{1}, \quad \sigma \otimes \sigma \cong \mathbb{1} \oplus \psi, \quad \psi \otimes \sigma \cong \sigma$,

which roughly describe how they behave when brought together in space. That is, two emergent fermions coalesce 
(a)

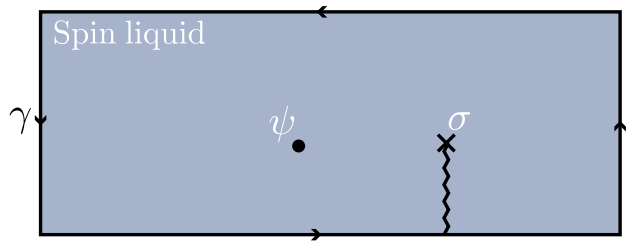

(b)
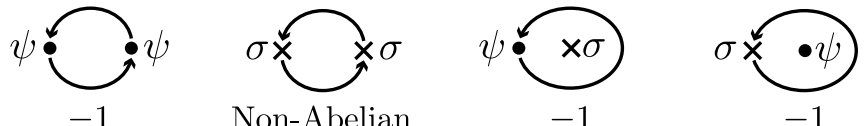

FIG. 2. Non-Abelian spin-liquid synopsis. (a) The boundary hosts a gapless chiral Majorana mode, while the bulk supports two nontrivial gapped quasiparticle types: emergent neutral fermions $\psi$ and Ising non-Abelian anyons $\sigma$. Fermions acquire a minus sign on crossing the wavy line (which represents a branch cut) emanating from $\sigma$. (b) Summary of quasiparticle braiding statistics.

into a local boson, two Ising anyons can combine to yield either a local boson or an emergent fermion, and Ising anyons can freely absorb emergent fermions without changing their quasiparticle type. Viewed "in reverse," a local boson can fractionalize into a pair of emergent fermions, an individual emergent fermion can further fractionalize into a pair of Ising anyons, and pairs of Ising anyons can be pulled out of the vacuum. Finally, $\psi$ and $\sigma$ particles exhibit not only nontrivial self-statistics, but also nontrivial mutual statistics: Taking a fermion all the way around an Ising anyon, or vice versa, yields a statistical phase of -1 . The above quasiparticle characteristics become essential for the circuits developed in Secs. VI and VII. Figure 2 summarizes the bulk and edge content of the non-Abelian spin liquid.

\section{PRIMER: INTERACTING HELICAL MAJORANA FERMIONS}

As an illuminating warm-up, next we explore gapless nonchiral-Majorana fermions propagating in 1D with strong interactions. We proceed in two stages: first examining interfaces between non-Abelian spin liquids, and then turning to one-dimensional lattice models that harbor similar physics. Results obtained here carry over straightforwardly to the quantum-Hall-spin-liquid interfaces that we introduce in Sec. IV and later exploit to electrically detect chiral Majorana edge states and bulk anyons in Kitaev materials (Secs. V through VII).

\section{A. Sewing up non-Abelian spin liquids}

Consider the setup from Fig. 3(a) consisting of two non-Abelian spin liquids realized in adjacent Kitaev materials. Physically, it is natural to anticipate that suitable (a)
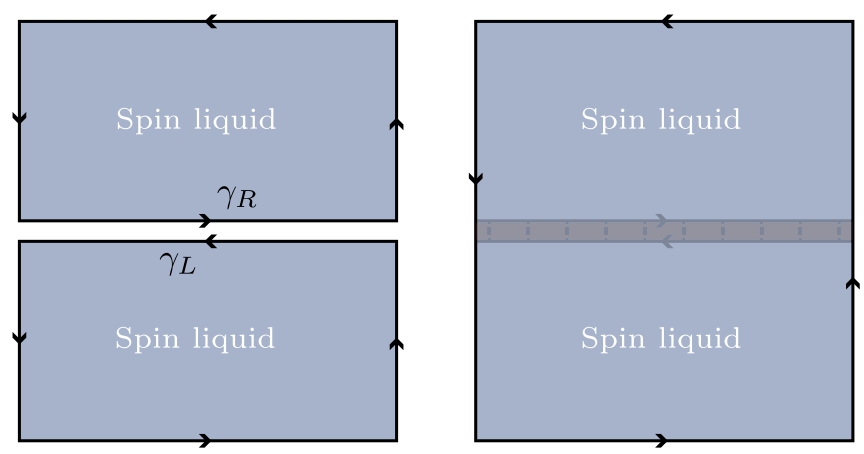

(c)

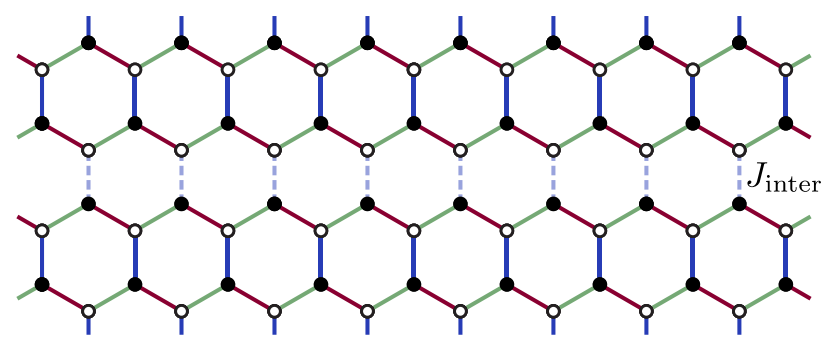

FIG. 3. (a) Decoupled non-Abelian spin liquids hosting emergent chiral Majorana fermions $\gamma_{R}$ and $\gamma_{L}$ at their interface. (b) Strong interactions between $\gamma_{R}$ and $\gamma_{L}$ [described by Eq. (17) with $\kappa>0$ ] gaps out these modes - thus sewing the two phases into a single non-Abelian spin liquid. (c) Microscopic view of the spin-liquid interface viewed as two Kitaev honeycomb models coupled via vertical bonds of strength $J_{\text {inter }}>0$ (dashed lines); see Eq. (19). The interface is fully gapped, as in (b), when $J_{\text {inter }}$ exceeds a critical value $J_{c}$, but otherwise hosts gapless Majorana modes, as in (a).

hybridization between the subsystems can effectively sew them together-producing a single, uninterrupted spin liquid. Our goal here is to understand this sewing-up process, both from effective field theory and microscopic viewpoints.

When the two layers decouple as in Fig. 3(a), their interface hosts helical Majorana modes whose kinetic energy is described by the low-energy Hamiltonian

$$
\mathcal{H}_{0}=\int_{x}\left(-i v \gamma_{R} \partial_{x} \gamma_{R}+i v \gamma_{L} \partial_{x} \gamma_{L}\right)
$$

Here, $x$ is a coordinate along the interface, $v$ is the edgestate velocity, and $\gamma_{R}$ and $\gamma_{L}$ respectively denote right- and left-moving Majorana-fermion fields. Upon turning on interactions between the adjacent layers, the Hamiltonian becomes $\mathcal{H}=\mathcal{H}_{0}+\delta \mathcal{H}$, where $\delta \mathcal{H}$ hybridizes the helical Majorana modes. Crucially, the form of $\delta \mathcal{H}$ is constrained by the fact that $\gamma_{R}$ and $\gamma_{L}$ represent emergent fermions originating from disjoint spin liquids. In particular, only pairs of emergent fermions-which together form a 


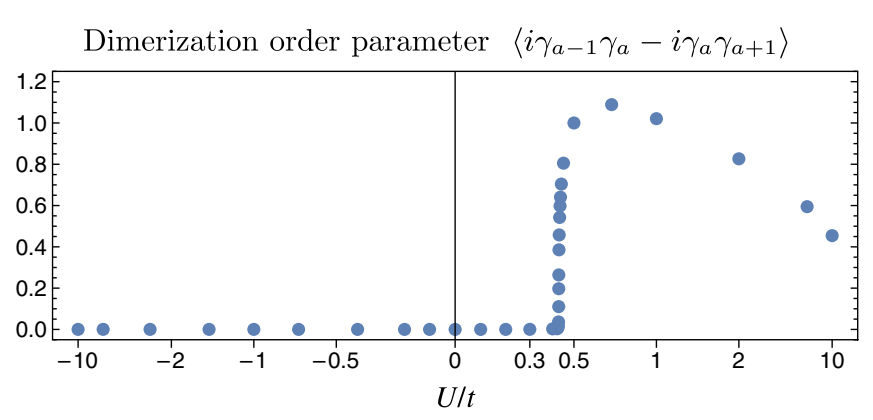

FIG. 4. Dimerization order parameter $\left\langle i \gamma_{a-1} \gamma_{a}-i \gamma_{a} \gamma_{a+1}\right\rangle$ versus $U / t$ obtained from DMRG simulations of the model in Eq. (20) with $t^{\prime}=0$. This order parameter corresponds to the fermion mass in the continuum limit. For $U / t \gtrsim 0.42799$ (2), our simulations capture a gapped, spontaneously dimerized phase for the chain, in agreement with Ref. [87]. At smaller $U / t$, the chain instead realizes a critical state with central charge $c=1 / 2$. We additionally verify that this critical state extends to large negative $U / t$ values.

boson-can tunnel across the interface. The simplest such interaction is given by

$$
\delta \mathcal{H}=-\kappa \int_{x}\left(\gamma_{R} \partial_{x} \gamma_{R}\right)\left(\gamma_{L} \partial_{x} \gamma_{L}\right)
$$

where the two derivatives are necessitated by Fermi statistics. Notice that $\mathcal{H}$ exhibits two independent $\mathbb{Z}_{2}$ symmetries, one corresponding to $\gamma_{R} \rightarrow-\gamma_{R}$ and the other corresponding to $\gamma_{L} \rightarrow-\gamma_{L}$. These symmetries can never be broken explicitly by any physical perturbation, reflecting the fact that individual Majorana fermions $\gamma_{R}$ and $\gamma_{L}$ live only within their respective spin liquids.

The coupling $\kappa$ is formally irrelevant at the fixed point described by the quadratic Hamiltonian $\mathcal{H}_{0}$. "Weak" $\kappa$ thus has only perturbative effects, and most importantly does not gap out the helical Majorana modes. Evidently, sewing up the spin liquids requires strong coupling. At "large" $\kappa>0$, the system can lower its energy by condensing $\left\langle i \gamma_{R} \gamma_{L}\right\rangle \neq 0$ - thereby spontaneously breaking the two independent $\mathbb{Z}_{2}$ symmetries noted above (but preserving their product). For rough intuition, consider the term $-\left(\kappa / \delta x^{2}\right)\left[i \gamma_{R}(x+\delta x) \gamma_{L}(x+\delta x)\right]\left[i \gamma_{R}(x) \gamma_{L}(x)\right]$, which upon Taylor expanding in the microscopic length $\delta x$ generates the interaction from Eq. (17). The discrete form above clearly reveals that $\left\langle i \gamma_{R} \gamma_{L}\right\rangle \neq 0$ is favored provided $\kappa$ is positive. In the condensed regime, the interface can be modeled by an effective mean-field Hamiltonian

$$
\mathcal{H}_{\mathrm{MF}}=\int_{x}\left(-i v \gamma_{R} \partial_{x} \gamma_{R}+i v \gamma_{L} \partial_{x} \gamma_{L}+i m \gamma_{R} \gamma_{L}\right)
$$

with $m \propto\left\langle i \gamma_{R} \gamma_{L}\right\rangle$ a mass whose sign, importantly, is chosen spontaneously. Equation (18) exhibits a fully gapped spectrum, and thus describes a scenario where (a)

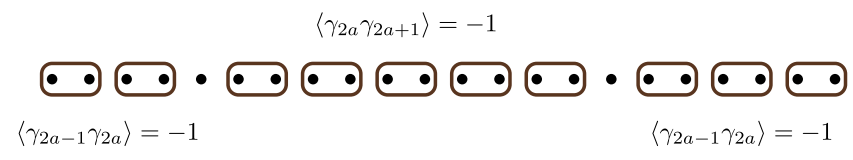

$m(x)$

(b)

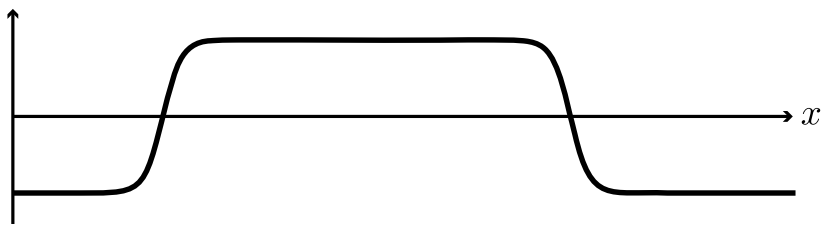

FIG. 5. (a) Snapshot of domain-wall excitations at the exactly solvable, spontaneously dimerized limit of Eq. (20) occurring for $t^{\prime}=0$ and $U=t / 2$. Neighboring sites $a, a+1$ enclosed by curved rectangles dimerize such that $\left\langle\gamma_{a} \gamma_{a+1}\right\rangle=-1$. Domain walls at which the dimerization pattern shifts by one site trap unpaired Majorana zero modes [nonenclosed dots in (a)]. In the low-energy limit, the dimerized chain is described at the meanfield level by Eq. (18), which includes a spontaneously generated mass $m$. Microscopic domain-wall excitations from (a) correspond in continuum language to excitations at which the mass $m$ changes sign, as depicted in (b). Such domain walls constitute 1D analogs of Ising anyons that arise at the spontaneously gapped spin-liquid interface of Fig. 3(b).

the two spin liquids have been sewn into one as sketched in Fig. 3(b).

Several consistency checks bolster the above picture. First, one can view the ground state $\left|\Psi_{\mathrm{MF}}\right\rangle$ of $\mathcal{H}_{\mathrm{MF}}$ as a trial wave function and the mass $m$ as a variational parameter. In Appendix A 1, we optimize $\left\langle\Psi_{\mathrm{MF}}\left|\mathcal{H}_{0}+\delta \mathcal{H}\right| \Psi_{\mathrm{MF}}\right\rangle$ with respect to $m$ for varying $\kappa$. This analysis indeed captures a nonzero mass $m$ provided the dimensionless ratio $\kappa \Lambda^{2} / v$ exceeds a critical value, where $\Lambda$ is an ultraviolet momentum cutoff. (For $\kappa<0$, the optimal mass always vanishes within this treatment.)

Second, the two nontrivial bulk anyons of the non-Abelian spin-liquid phase are encoded in the simple mean-field Hamiltonian $\mathcal{H}_{\mathrm{MF}}$ describing the gapped interface [86]. Neutral fermions are clearly present as gapped excitations. Ising non-Abelian anyons form at domain walls in which the spontaneously chosen mass $m$ changes sign; see Fig. 5(b) for an illustration. Unpaired Majorana zero modes bind to such domain walls, leading to the hallmark degeneracy associated with Ising anyons. Furthermore, since the sign of the mass is arbitrary, separating the domain walls by arbitrary distances costs only finite energy - i.e., the Ising anyons are bona fide deconfined quasiparticles. Additional insights into the domain-wall structure can be gleaned from the lattice model that we discuss in Sec. III B.

Third, the low-energy perspective that we present above seamlessly connects to microscopics. Let us add a spin-spin interaction 


$$
\delta H=-J_{\text {inter }} \sum_{\left(\mathbf{r}, \mathbf{r}^{\prime}\right)} S_{\mathbf{r}^{\prime}}^{z} S_{\mathbf{r}^{\prime}}^{z}
$$

that couples spins across bonds $\left(\mathbf{r}, \mathbf{r}^{\prime}\right)$ [dashed lines in Fig. 3(c)] that bridge the adjacent spin liquids. At $J_{\text {inter }}=0$, one recovers decoupled spin liquids, and the interface hosts gapless Majorana modes that are stable to weak perturbations. On symmetry grounds, the boundary spin operators relate to continuum Majorana fields via $S_{\mathbf{r}}^{z} \sim\left\langle S_{\mathbf{r}}^{z}\right\rangle+$ $\alpha: i \gamma_{R} \partial_{x} \gamma_{R}:$ on the lower edge of the interface and $S_{\mathbf{r}}^{z} \sim$ $\left\langle S_{\mathbf{r}}^{z}\right\rangle-\alpha: i \gamma_{L} \partial_{x} \gamma_{L}$ : on the upper edge. Here, angle brackets indicate ground-state expectation values, $\alpha$ is a nonuniversal constant, and : : denotes normal ordering; the relative minus sign in the $\alpha$ pieces above reflects the opposite chirality for the two modes. Using this continuum expansion, the microscopic interaction $\delta H$ indeed generates the effective-Hamiltonian term in Eq. (17) with $\kappa \propto J_{\text {inter }}$. At $J_{\text {inter }}=J$-corresponding to the strong-coupling limitthe system forms a single, translationally invariant nonAbelian spin liquid; here, all gapless modes at the interface have clearly been vanquished. It follows that the spin liquids are sewn up provided $J_{\text {inter }}$ exceeds a critical value $J_{c}$ that satisfies $0<J_{c}<J$, in qualitative agreement with our continuum analysis.

\section{B. Insights from microscopic models}

Complementary insights can be gleaned by examining a strictly 1D toy lattice model that also realizes interacting helical Majorana fermions. Consider an infinite chain of physical (rather than emergent) Majorana fermions $\gamma_{a}$ living on lattice sites $a$ and governed by the microscopic Hamiltonian

$H=\sum_{a}\left(i t \gamma_{a} \gamma_{a+1}+i t^{\prime} \gamma_{a} \gamma_{a+2}-U \gamma_{a-2} \gamma_{a-1} \gamma_{a+1} \gamma_{a+2}\right)$

we take $t>0$ for concreteness throughout. References [87, 88 ] recently studied this model motivated in part by interesting connections to supersymmetry; see also Ref. [89]. Importantly, $H$ preserves an (anomalous [90]) translation symmetry $T$ that transforms $\gamma_{a} \rightarrow \gamma_{a+1}$. At $t^{\prime}=0$, the Hamiltonian further preserves an antiunitary chiral symmetry $\mathcal{C}$ that sends $\gamma_{a} \rightarrow(-1)^{a} \gamma_{a}$ and $i \rightarrow-i$ [91].

We first specialize to $t^{\prime}=0$. In the $U=0$ limit the chain is gapless. Here, one can capture the low-energy physics by writing

$$
\gamma_{a} \sim \gamma_{L}+(-1)^{a} \gamma_{R}
$$

where $\gamma_{R}$ and $\gamma_{L}$ again denote right- and left-moving Majorana fields, leading precisely to Eq. (16) with $v \propto t$. Translation symmetry $T$ sends $\gamma_{R} \rightarrow-\gamma_{R}$ (just as for one of the $\mathbb{Z}_{2}$ symmetries present for the spin-liquid interface examined above), while $\mathcal{C}$ swaps $\gamma_{R} \leftrightarrow \gamma_{L}$. A mass term $i m \gamma_{R} \gamma_{L}$ is odd under $T$, and thus can never be generated explicitly by any $T$-preserving perturbation, similar to the scenario encountered in Sec. III A.

Upon restoring nonzero $U$, the leading term that couples right and left movers corresponds to Eq. (17) with $\kappa \propto U$ [87]. Previous density-matrix renormalization-group (DMRG) simulations of Eq. (20) (which we reproduce and extend to include $U<0$ in Fig. 4) indicate that for $U \gtrsim 0.428 t$, criticality is destroyed in favor of a gapped, dimerized phase that spontaneously breaks $T$ symmetry [87]. In continuum language, here $\left\langle i \gamma_{R} \gamma_{L}\right\rangle \neq 0$ condenses and the helical Majorana fermions are gapped via generation of a mass $m \propto\left\langle i \gamma_{R} \gamma_{L}\right\rangle$ with arbitrary sign. Appendix A 2 analyzes Eq. (20) at $t^{\prime}=0$ using a variational approach that predicts spontaneous dimerization for $U \gtrsim 0.295 t$, in rough agreement with DMRG.

The gapped ground states at $U=t / 2$ are known exactly [87] and can be recovered by postulating "perfect" dimerization with $\mathcal{O} \equiv\left\langle i \gamma_{2 a} \gamma_{2 a+1}\right\rangle=-1$ and $\left\langle i \gamma_{2 a-1} \gamma_{2 a}\right\rangle=0$. Decoupling the $U$ term using this ansatz generates a meanfield Hamiltonian $H_{\mathrm{MF}}=t \sum_{a} i \gamma_{2 a} \gamma_{2 a+1}$ for which $\mathcal{O}=$ -1 in the ground state-indicating self-consistency. One can similarly show that the shifted dimerization with $\left\langle i \gamma_{2 a-1} \gamma_{2 a}\right\rangle=-1$ and $\left\langle i \gamma_{2 a} \gamma_{2 a+1}\right\rangle=0$ yields a degenerate self-consistent solution. Using Eq. (21), we have

$$
\left\langle i \gamma_{a} \gamma_{a+1}\right\rangle \sim \text { const }+(-1)^{a}\left\langle i \gamma_{R} \gamma_{L}\right\rangle
$$

it follows that these two dimerizations correspond to opposite-sign masses in the continuum formulation.

In Sec. III A, we observe that the interface between two sewn-up spin liquids [Fig. 3(b)] supports gapped emergent fermionic excitations, and that domain walls at which the mass $m$ changes sign correspond to Ising anyons hosting unpaired Majorana zero modes. The above mean-field ansatz at $U=t / 2$, though operative in a physical-fermion system, provides an intuitive picture for these fractionalized quasiparticles [92]. The mean-field construction suggests that low-energy states can be labeled by domains exhibiting fixed dimerization-either $\left\langle i \gamma_{2 a-1} \gamma_{2 a}\right\rangle=-1$ or $\left\langle i \gamma_{2 a} \gamma_{2 a+1}\right\rangle=-1$-along with fermionic excitations within a given domain. Fermionic excitations arise from flipping the sign of the dimerization expectation value at a particular bond, e.g., replacing $\left\langle\gamma_{2 b-1} \gamma_{2 b}\right\rangle \rightarrow+1$ for some $b$. Figure 5(a) illustrates an excited configuration with domain walls separating the two dimerization patterns, while Fig. 5(b) shows the corresponding sign-changing mass profile in the continuum description. The domain walls clearly harbor unpaired Majorana zero modes as a consequence of the dimerization shift. Pairs of domain walls share a pair of Majorana zero modes, and therefore nonlocally host a single complex fermionic mode. The occupancy of this complex fermion dictates whether the pair of domain walls fuse to the local vacuum or a fermion. Comparing these quantum states to those at the interface of Fig. 3(b), we can identify domain walls in the 1D model 
with Ising anyons in the spin liquid, and the excitations to which they fuse as the local vacuum or the emergent fermion.

More technically, we can also use this illustration to relate the ground-state degeneracy of Eq. (20) with open boundary conditions to the ground-state degeneracy of the spin liquid on a cylinder. For this purpose, we identify the 1D chain governed by Eq. (20) with the degrees of freedom along a path that connects the upper and lower cylinder ends. We can then view $U$ as a tuning potential that slices open or resews the cylinder as $U$ passes below or above the critical interaction strength at which spontaneous mass generation occurs. It is known that a topologically ordered phase on a cylinder exhibits a ground-state degeneracy given by the number of bulk anyons: three in the nonAbelian spin liquid of interest here (corresponding to anyons $\mathbb{1}, \sigma$, and $\psi$ ). Hence, we expect the gapped phase of Eq. (20) to also admit three ground states, which is indeed the case as can be seen readily at $U=t / 2$. In this limit, two of the ground states arise from the dimerization pattern that yields an unpaired Majorana at each end of the open chain; these states, which we label $|\mathbb{1}\rangle$ and $|\psi\rangle$, can be identified with $\mathbb{1}$ and $\psi$ anyons. The third arises from the shifted dimerization wherein the chain is fully gapped, including at the ends; this state denoted $|\sigma\rangle$ can be identified with $\sigma$. As a sanity check, we can pass between the two dimerization patterns by nucleating a pair of domain walls in the bulk of the chain and then bringing one to each boundary. If the chain begins in $|\mathbb{1}\rangle$ or $|\psi\rangle$, the boundary Majorana zero modes pair up with those carried by the domain walls and create $|\sigma\rangle$ (or a locally related excited state). Conversely, if the chain begins in $|\sigma\rangle$, the domain walls shuttle unpaired Majorana zero modes to the boundary and thus yield $|\mathbb{1}\rangle$ or $|\psi\rangle$.

Next, we restore $t^{\prime} \neq 0$. At $U=0$, the chain remains gapless, though the velocities $v_{L}$ and $v_{R}$ for left and right movers now differ due to the loss of $\mathcal{C}$ symmetry. Explicitly, we have

$$
\frac{v_{R}}{v_{L}}=\frac{t-2 t^{\prime}}{t+2 t^{\prime}},
$$

which vanishes as $t^{\prime} \rightarrow t / 2$. (For $t^{\prime}>t / 2$, additional lowenergy modes appear; we consider only $0 \leq t^{\prime}<t / 2$ here.) Reference [87] found that velocity anisotropy very weakly influences the critical interaction $U$ above which the chain spontaneously dimerizes. Our DMRG simulations confirm this result: The critical $U$ shifts by less than $0.6 \%$ for all $v_{R} / v_{L}>0$. For instance, we find $U_{c} / t \approx 0.4302(1)$ as $t^{\prime} / t \rightarrow 0.5$, compared to $U_{c} / t \approx 0.42799(2)$ at $t^{\prime}=0$. Figure 6 summarizes the phase diagram extracted from DMRG.

A spontaneously dimerized gapped phase can also arise in the modified model obtained by replacing the fourfermion interaction in Eq. (20) with $U_{R} \gamma_{a-1} \gamma_{a} \gamma_{a+1} \gamma_{a+2}$.

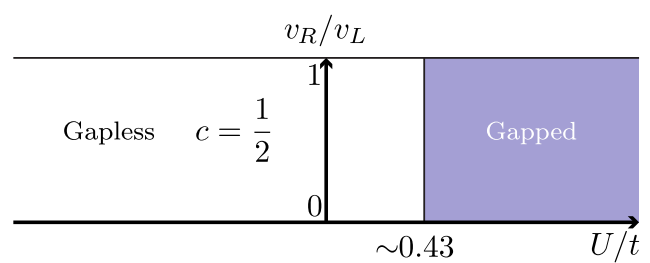

FIG. 6. Schematic phase diagram of Eq. (20) as a function of $U / t$ and velocity anisotropy induced by $t^{\prime}$. Here, $v_{R}$ and $v_{L}$, respectively, denote the velocities for right- and left-moving Majorana fermions at $U=0$; Eq. (23) specifies their ratio. We assume $0<v_{R} / v_{L} \leq 1$ for simplicity, though clearly the same physics arises when $v_{R} \leftrightarrow v_{L}$. Crucially, the phase boundary separating the critical $c=1 / 2$ state and the spontaneously dimerized gapped phase depends very weakly on $v_{R} / v_{L}$-as we find in our DMRG calculations. This observation suggests that unequal Majorana-fermion velocities, which would be expected for right and left movers of distinct physical origin (see, e.g., Fig. 7), do not obstruct the formation of a gapped phase.

This seemingly innocuous microscopic modification, however, boosts the required interaction strength by 3 orders of magnitude: $U_{R} \gtrsim 250 t[93,94]$. In Appendix B, we explain this curious observation (among other aspects of this model's phase diagram) as arising from kinetic energy renormalization by the $U_{R}$ interaction. In continuum language, increasing $U_{R}$ both generates the interaction in Eq. (17) and increases the velocity in Eq. (16), thereby sharply suppressing the onset of the strong-coupling limit where interactions dominate over kinetic energy.

\section{SEWING A NON-ABELIAN SPIN LIQUID TO AN ELECTRONIC QUANTUM HALL PHASE}

We have now seen two examples wherein strong interactions catalyze a condensation transition with $\left\langle i \gamma_{R} \gamma_{L}\right\rangle \neq 0$. At the interface between two non-Abelian spin liquids examined in Sec. III A, $\gamma_{R}$ and $\gamma_{L}$ both represent emergent fermions residing in initially separate fractionalized bosonic systems that are stitched together by the condensation. In the strictly 1D model from Sec. III B, by contrast, both fields represent physical fermions. Next, we explore a system in which a very similar condensation arises, but instead from the combination of a physical and emergent Majorana fermion-providing a means of coherently converting one into the other.

Figure 7(a) illustrates the setup consisting of an electronic integer quantum-Hall system at filling factor $\nu=1$ adjacent to a non-Abelian spin liquid. Additionally, the quantum-Hall edge couples to a conventional superconductor; here and in similar setups that we study in later sections, we assume fully gapped superconductivity, though we briefly discuss the role of low-lying excitations deriving from vortices and/or disorder in Sec. VIII. We first present a qualitative picture for the physics that arises from interactions between these subsystems. 
(a)
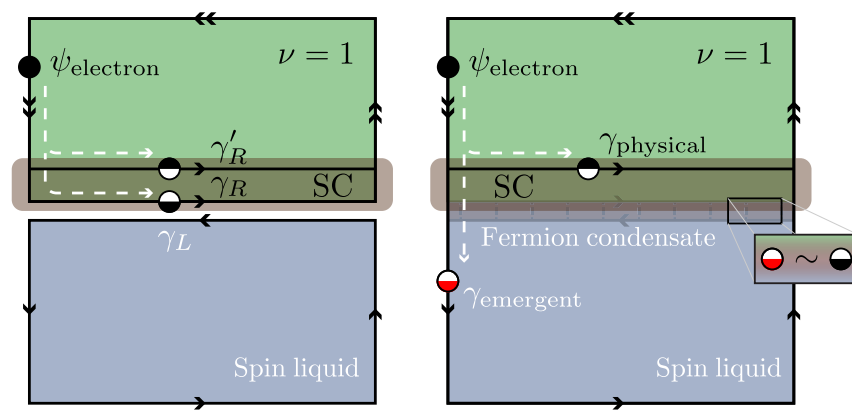

FIG. 7. (a) Interface between a non-Abelian spin liquid and a proximitized $\nu=1$ integer quantum-Hall system. The bare $\nu=1$ edge state-indicated by double arrows-generically separates into two copropagating Majorana fermions $\gamma_{R}$ and $\gamma_{R}^{\prime}$ beneath the superconductor (SC) in the central region. (b) Strong interactions can gap out $\gamma_{R}$ and the spin liquid's edge Majorana fermion $\gamma_{L}$ via formation of a fermion condensate that partially sews the two subsystems together. Physical Majorana fermions (black halfcircles) and emergent Majorana fermions (red half-circles) are then identified at the interface; see enlargement for an illustration. A striking consequence follows: An electron injected at low energies into the $\nu=1$ edge splinters into a pair of Majorana fermions, one of which unavoidably enters the spin liquid.

The quantum-Hall edge hosts a chiral mode that can be viewed as a pair of copropagating chiral Majorana fermions (hence, double arrows employed in our illustrations). Beneath the superconductor, the loss of charge conservation generically allows those copropagating Majorana fermions to displace from one another as shown in Fig. 7(a). Microscopic interactions can backscatter only electrically neutral bosons-e.g., energy-between the quantum-Hall and spin-liquid edge states because the latter edge modes reside in an electrical insulator that hosts only emergent fermions. More precisely, a charge-2ne excitation ( $n$ is an integer) from the quantum-Hall edge can neutralize by shedding its charge into the superconducting condensate [95], and then backscatter into the spin-liquid edge mode. Sufficiently strong backscattering events of this nature partially sew up the quantum-Hall state and non-Abelian spin liquid. That is, the emergent chiral Majorana fermion from the latter spin-liquid edge gaps out with "half" of the $\nu=1$ edge mode, leaving a single physical chiral Majorana edge mode behind. As sketched in Fig. 7(b), an electron injected at low energies into the "naked" part of the quantum-Hall edge then splinters into a pair of Majorana fermions, one of which unavoidably enters the non-Abelian spin liquid as an emergent fermion.

For a more formal analysis, we write the effective Hamiltonian for the interface as

$$
\mathcal{H}=\mathcal{H}_{\mathrm{SL}}+\mathcal{H}_{\mathrm{QH}}+\delta \mathcal{H}
$$

The first term

$$
\mathcal{H}_{\mathrm{SL}}=\int_{x}\left(i v_{L} \gamma_{L} \partial_{x} \gamma_{L}\right)
$$

describes the spin liquid's emergent chiral Majorana edge state with velocity $v_{L}$. The second governs the proximitized $\nu=1$ edge and takes the form

$\mathcal{H}_{\mathrm{QH}}=\int_{x}\left[-i u_{R} \psi_{R}^{\dagger} \partial_{x} \psi_{R}+\Delta\left(i \psi_{R} \partial_{x} \psi_{R}+\right.\right.$ H.c. $\left.)\right]$,

where $\psi_{R}$ is a complex fermion operator that removes electrons from the edge state, $u_{R}$ is the associated velocity, and $\Delta$ is the proximity-induced pairing amplitude. Passing to a Majorana representation via $\psi_{R}=\gamma_{R}+i \gamma_{R}^{\prime}$, one can equivalently write [96]

$$
\mathcal{H}_{\mathrm{QH}}=\int_{x}\left(-i v_{R} \gamma_{R} \partial_{x} \gamma_{R}-i v_{R}^{\prime} \gamma_{R}^{\prime} \partial_{x} \gamma_{R}^{\prime}\right)
$$

the velocities for the constituent copropagating Majorana fermions $\gamma_{R}$ and $\gamma_{R}^{\prime}$ are $v_{R}=u_{R}-2 \Delta$ and $v_{R}^{\prime}=u_{R}+2 \Delta$, respectively.

The final term in Eq. (24) encodes interactions between the spin-liquid and quantum-Hall edge states. Suppose that $\gamma_{R}$ resides closest to $\gamma_{L}$ as in Fig. 7. It is then reasonable to assume that interactions predominantly couple these fermions, so we take $\delta H$ to be given precisely by Eq. (17). Useful insight follows from reexpressing $\delta H$ in terms of complex fermions $\psi_{R}: \delta H=-\frac{1}{4} \kappa \int_{x}\left(\gamma_{L} \partial_{x} \gamma_{L}\right)\left(\psi_{R}^{\dagger} \partial_{x} \psi_{R}+\right.$ $\psi_{R} \partial_{x} \psi_{R}+$ H.c.). Here we see that interactions transfer electrically neutral dipoles as well as Cooper pairs from the quantum-Hall edge to the spin liquid, consistent with our preceding physical picture.

The full Hamiltonian in Eq. (24) reduces to the model studied in the previous subsections, supplemented by a decoupled sector for the $\gamma_{R}^{\prime}$ chiral Majorana fermion. We immediately conclude that strong interactions can condense $\left\langle i \gamma_{R} \gamma_{L}\right\rangle \neq 0$ and partially gap out the interface, again in line with the above physical picture. This conclusion holds even if the velocities $v_{L}$ and $v_{R}$-which bear no relation-differ significantly; recall Sec. III B.

In the present context, the transition to a state with $\left\langle i \gamma_{R} \gamma_{L}\right\rangle \neq 0$ is sometimes referred to as fermion condensation since the condensed object involves only one physical fermion (specifically, $\gamma_{R}$ ). A precise mathematical formulation of this striking phenomenon can be found in Ref. [76]; see also Refs. $[73,74,97]$ for related earlier applications. The essential role played by proximity-induced superconductivity also becomes clear from this vantage point. The condensate $\left\langle i \gamma_{R} \gamma_{L}\right\rangle \sim\left\langle i\left(\psi_{R}+\psi_{R}^{\dagger}\right) \gamma_{L}\right\rangle \neq 0$ clearly does not preserve $\mathrm{U}(1)$ charge conservation for the physical fermions. Without externally imposed superconductivity, interactions would thus need to spontaneously break U(1) in order to partially gap the interface, which cannot transpire due to the quasi-1D nature of the interface. Finally, we note that 
essentially the same fermion condensation transition can arise from interfacing a non-Abelian spin liquid with other electronic platforms, including conventional spinful 1D wires. We discuss alternative setups further in Sec. VIII.

\section{ELECTRICAL DETECTION OF CHIRAL MAJORANA EDGE MODES IN NON-ABELIAN SPIN LIQUIDS}

As a first application of the phenomena that we develop in Sec. IV, we introduce a scheme for electrically detecting the spin liquid's emergent chiral Majorana edge state. Figure 8(a) sketches the relevant circuit. Here, a pair of $\nu=1$ quantum-Hall systems flank a non-Abelian spin liquid. The interfaces are partially gapped by fermion condensation, facilitated by superconductors that are floating but exhibit negligible charging energy; note that the superconductors connect on the bottom end. A source on the far left is biased with voltage $V$, while a drain on the far right is grounded. We stress that no electrical current flows through spin liquid-which still realizes a good Mott insulator. Any current instead passes between the quantum-Hall systems via the intervening floating superconductor in the form of Cooper pairs. Nevertheless, the spin liquid is by no means a spectator: Electrons from the source propagate chirally along the $\nu=1$ edge, then partially convert into emergent chiral Majorana fermions in the spin liquid, and finally reenter the $\nu=1$ edge as physical fermions on the other end. This inevitable conversion between physical and emergent fermions ultimately dictates the circuit's electrical transport characteristics as we will see.

We focus on vanishingly small temperature $T$ and bias voltage $V$, where the conductance attains a universal quantized value of $G=\left(e^{2} / 2 h\right)$. To understand this value, first observe that the left and right halves constitute identical resistors in series; hence, $G=g / 2$ with $g$ the conductance for (say) the left half. One can deduce $g$ as follows. An incident electron from the source splits up into an emergent Majorana fermion in the spin liquid and a physical Majorana fermion that reflects back to the source [see Fig. 8(a)]. The physical Majorana fermion is, by definition, equal part electron and equal part hole, implying probability $1 / 2$ for Andreev reflection. Thus, $g=\frac{1}{2} \times\left(2 e^{2} / h\right)$, where the factor of 2 in the numerator arises because each Andreev process injects a Cooper pair into the superconductor. The overall conductance is then $G=\left(e^{2} / 2 h\right)$ as advertised. Although we focus on $V \rightarrow 0$ here, the conductance remains $G \approx\left(e^{2} / 2 h\right)$ for voltages below the gap scale of the fermion condensate.

What happens if the emergent chiral Majorana fermions disappear entirely from the setup? One can readily arrange this scenario, e.g., by changing the magnetic field such that the Kitaev material exits the non-Abelian spin-liquid phase. The resulting circuit-which furnishes an essential control

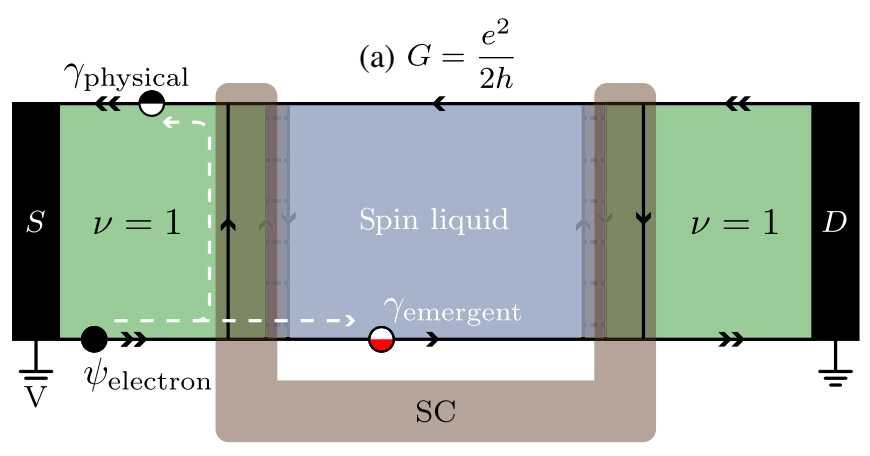

(b) $G=0$

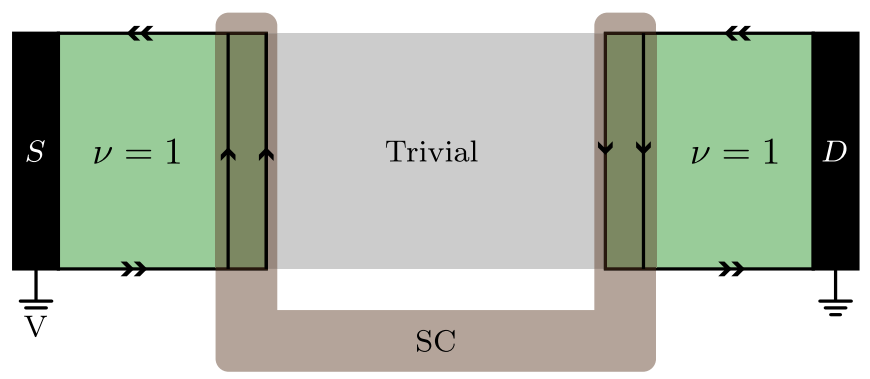

FIG. 8. (a) Circuit that electrically detects the spin liquid's emergent chiral Majorana edge state. Any electrical current that flows between the source $(S)$ and drain $(D)$ necessarily passes through the central superconductor (and not the spin liquid). Nevertheless, the perfect conversion between physical and emergent fermions at the quantum-Hall-spin-liquid interfaces dictates the conductance-which at zero bias is quantized at $G=\left(e^{2} / 2 h\right)$. (b) Control circuit in which the spin liquid is replaced with a trivial phase (e.g., a magnetically ordered state). Elimination of the physical-fermion $\leftrightarrow$ emergent-fermion conversion processes that underlie transport in (a) leads to vanishing zero-bias conductance $G=0$ in this case.

experiment-appears in Fig. 8(b). Most importantly, fermion condensation is now precluded, so that both $\nu=1$ edge states must simply "turn around" at the interface with the central trivial region. In this case, the probability for Andreev reflection vanishes at asymptotically low energies, yielding conductance $G=0$.

The absence of Andreev processes can be most simply understood as a consequence of Fermi statistics: The induced pairing term for the $\nu=1$ edge state $\Delta\left(i \psi_{R} \partial_{x} \psi_{R}+\right.$ H.c. $)$ necessarily contains a derivative and thus vanishes with the incident electron's momentum. Alternatively, as the two copropagating Majorana fermions traverse the superconductor, they generally acquire different phase factors, in turn "rotating" the incident electron in particle-hole space and generating Andreev processes. The phase difference explicitly reads $\delta \phi=\left(k-k^{\prime}\right) L$, where $k$ and $k^{\prime}$ denote the wave vectors of the two Majorana fermions as they pass through the superconducting region of length $L$. At finite incident energy, the wave vectors differ, i.e., $k \neq k^{\prime}$, due to unequal velocities for the Majorana fermions in that region; recall 
Eq. (27). As the incident-electron energy vanishes, however, $k, k^{\prime} \rightarrow 0$ and hence, $\delta \phi \rightarrow 0$ [98]. An incoming electron must then exit the superconductor as an electron, yielding zero net current across the circuit in Fig. 8(b). In this control scenario the conductance remains $G \approx 0$ up to voltages $V \sim(1 / e L)\left(v_{R} v_{R}^{\prime} /\left|v_{R}-v_{R}^{\prime}\right|\right)$, at which $\delta \phi$ becomes appreciable [96].

The contrast between the two circuits in Fig. 8 is particularly striking given that they differ solely in the properties of an electrically inert element. Nontrivial conductance quantization for Fig. 8(a) relies on emergent chiral Majorana fermions in the non-Abelian spin liquid together with fermion condensation, and thus constitutes an electrical signature of both phenomena. As an aside, local heat capacity measurements at the quantum-Hall-spinliquid interface may serve as a complementary probe of fermion condensation. The local heat capacity per unit length is $c_{v}=\left(\pi^{2} k_{B}^{2} T / 3 h v\right)\left(c_{L}+c_{R}\right)$, where $c_{L}$ and $c_{R}$ denote the central charges for the "left" and "right" movers at the interface. Decoupled edges yield $c_{L}+c_{R}=3 / 2$, while fermion condensation reduces the sum to $c_{L}+c_{R}=$ $1 / 2$ via partial gapping of the interface. Note, however, that backing out the central charges from this approach requires (rough) knowledge of the edge velocity $v$.

Figure 8(a) closely resembles the quantum anomalous Hall-superconductor heterostructures studied theoretically in Refs. [30,99-104] and experimentally in Ref. [105], where precisely the same quantized conductance was proposed as a signature of physical chiral Majorana edges states at the boundary of a two-dimensional topological superconductor. In that context, alternative quantization mechanisms that do not invoke chiral Majorana modes have also been introduced (e.g., disorder and dephasing, or if the superconductor behaves as a normal contact [106108]; see also the critical discussion in Ref. [109]). If operative in our setups, such trivial mechanisms would-at most-depend weakly on the precise phase of matter realized in the Kitaev material, thus yielding similar transport characteristics for both circuits in Fig. 8. Observing the qualitatively different conductances predicted for Figs. 8(a) and 8 (b) would therefore strongly suggest against these alternative interpretations.

We now briefly discuss the feasibility of the experiment shown in Fig. 8. We require a superconductor that can survive the high magnetic fields necessary to bring the left and right sides into the quantum-Hall regime, and in particular into the $\nu=1$ state. Let us consider for concreteness a structure that consists of monolayer graphene in the quantum-Hall regime, coupled on both sides to $\alpha-\mathrm{RuCl}_{3}$. The magnetic field necessary to bring $\alpha-\mathrm{RuCl}_{3}$ into the gapped spin-liquid state is roughly $10 \mathrm{~T}$ applied at $45^{\circ}$ to the plane, resulting in a 7-T out-of-plane field and 7-T in-plane field [60]. This field scale is advantageous for the graphene $\nu=1$ state, which is readily attainable in clean samples at fields much lower than $7 \mathrm{~T}$, and is stabilized by a large in-plane field because it is a spinpolarized state [110]. Nearly transparent contacts have been made between superconducting $\operatorname{MoRe}\left(H_{c 2} \sim 10 \mathrm{~T}\right)$ and graphene in experiments designed specifically to study the interface between superconductors and quantum-Hall edge states (including the $\nu=1$ state) $[111,112]$. Higher out-ofplane critical fields could be attained by using $\mathrm{NbN}$ or $\mathrm{NbTiN}$, both of which have been used to make contact to graphene [113,114] and have $H_{c 2}$ exceeding $10 \mathrm{~T}$, or even by integrating a layered van der Waals superconductor such as $\mathrm{FeSe}_{x} \mathrm{Te}_{1-x}$, which can have $H_{c 2}$ in excess of $28 \mathrm{~T}$ at $T=0[115]$.

\section{ELECTRICAL DETECTION OF BULK ISING NON-ABELIAN ANYONS}

One can also employ electrical transport to detect individual bulk Ising anyons in a non-Abelian spin liquid - in fact using a slightly simpler circuit compared to Fig. 8. Consider now the setups shown in Fig. 9. There, a single $\nu=1$ quantum-Hall system is partially sewn to a spin liquid via fermion condensation mediated by a grounded superconductor. A source at bias voltage $V$ on the left generates a current $I$ that flows through the superconductor to ground. More precisely, electrons emanating from the source (i) propagate along the lower $\nu=1$ edge, then (ii) fragment into emergent and physical Majorana fermions that acquire a phase difference $\delta \phi$ upon encircling the spin liquid, and finally (iii) recombine into either electrons, holes, or superpositions thereof depending on $\delta \phi$. Recombination into a hole in this final step indicates absorption of a Cooper pair into the superconductor, thereby contributing to the current $I$. We are interested in the conductance $G=d I / d V$ in the limit $T, V \rightarrow 0$ (but see the next section for an extension to finite $V$ ). Just as we see in Sec. V, the spin liquid-although electrically inertplays a decisive role in electrical transport: One of two distinct universal quantized conductances emerges depending on the quasiparticle configuration in the spin liquid.

Suppose first that the spin liquid's interior is devoid of Ising non-Abelian anyons as in Fig. 9(a). Here, the phase difference acquired in stage (ii) is simply

$$
\delta \phi=k_{p} L_{p}-k_{e} L_{e},
$$

with $k_{p}$ the momentum of the physical Majorana fermion as it travels the distance $L_{p}$ between the lower and upper $\nu=1$ edge, and $k_{e}$ and $L_{e}$ the analogous quantities for the emergent Majorana fermion. The limit $k_{p}, k_{e} \rightarrow 0$ yields $\delta \phi \rightarrow 0$, implying that at asymptotically low energies, incident electrons recombine into outgoing electrons with unit probability. To summarize, stages (i) through (iii) proceed according to

$$
\psi_{\text {electron }} \rightarrow \gamma_{\text {physical }}+i \gamma_{\text {emergent }} \rightarrow \psi_{\text {electron }},
$$


(a) $G=0$

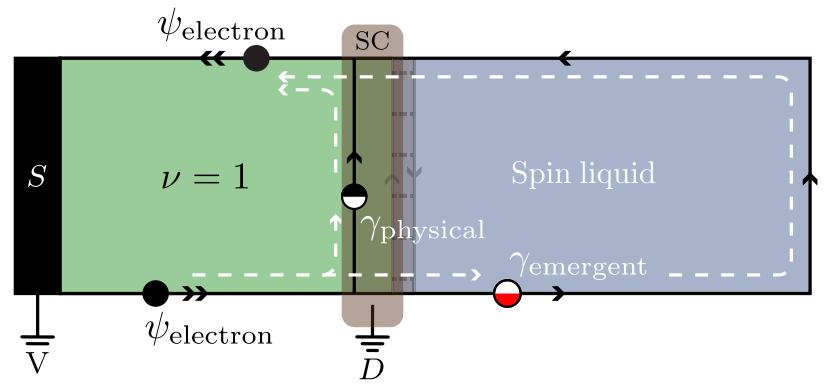

(b) $G=\frac{2 e^{2}}{h}$

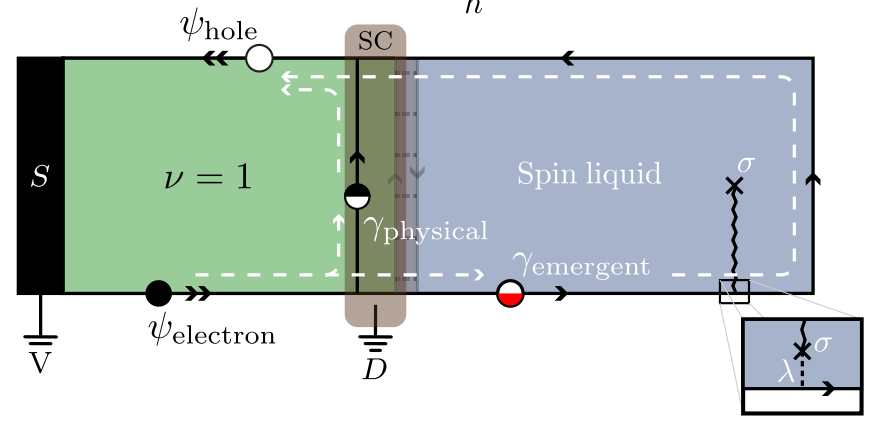

(c)

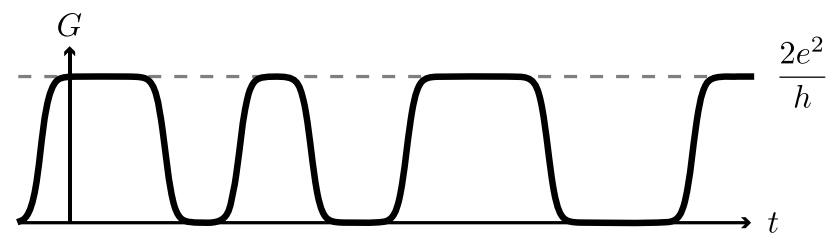

FIG. 9. (a),(b) Electrical detection of bulk Ising anyons via quantized zero-bias conductance $G$. Current flows from the source to the grounded superconductor. In (a), the spin liquid contains no Ising anyons in the bulk. Here, electrons injected at zero energy along the lower $\nu=1$ edge splinter into physical and emergent Majorana fermions, but simply recombine into electrons at the upper $\nu=1$ edge. No current flows into the superconductor and hence, $G=0$. In (b), a pair of bulk Ising anyons is pulled out of the vacuum, with one of those anyons dragged to the gapless edge. Nontrivial mutual statistics between emergent fermions and the single remaining bulk Ising anyon causes incident zero-energy electrons to recombine perfectly into holes at the upper $\nu=1$ edge. Each injected electron transmits a Cooper pair into the superconductor, yielding $G=\left(2 e^{2} / h\right)$. Enlargement: The Ising anyon dragged to the boundary couples to the chiral Majorana edge state with strength $\lambda$ [see Eq. (32)]. (c) If random thermal processes toggle the system between configurations (a) and (b), $G$ exhibits telegraph noise as a function of time, switching stochastically between $G=0$ and $\left(2 e^{2} / h\right)$.

as Fig. 9(a) illustrates. No current flows into the superconductor, and therefore, $G=0$. Note the similarity to the physics encountered for the control circuit in Fig. 8(b).

Next, imagine nucleating a pair of Ising non-Abelian anyons and then dragging one of those anyons to a gapless part of the spin-liquid edge [116]. The resulting setup shown in Fig. 9(b) contains a single Ising anyon in the bulk. At asymptotically low incident-electron energies, the emergent Majorana fermion in stage (ii) acquires an additional minus sign upon crossing the Ising anyon absorbed at the edge [i.e., at the termination of the wavy line in the inset of Fig. 9(b); see below for further details]. This allimportant minus sign reflects the nontrivial mutual statistics between emergent fermions and Ising anyons in the spin liquid (recall Sec. II B). It follows that $\delta \phi \rightarrow \pi$ as $k_{p}$, $k_{e} \rightarrow 0$, implying that at low energies, incident electrons recombine into outgoing holes with unit probability. Stages (i) through (iii) can then be summarized as

$$
\begin{aligned}
\psi_{\text {electron }} & \rightarrow \gamma_{\text {physical }}+i \gamma_{\text {emergent }} \\
& \rightarrow \gamma_{\text {physical }}-i \gamma_{\text {emergent }} \rightarrow \psi_{\text {hole }}
\end{aligned}
$$

see Fig. 9(b). The perfect "Andreev conversion" of electrons into holes yields nontrivially quantized conductance $G=\left(2 e^{2} / h\right)$.

More generally, if the fragmented emergent and physical Majorana fermions encircle $n_{\sigma}$ Ising anyons in the interior of the spin liquid, the phase difference at low energies is $\delta \phi=\pi n_{\sigma}$, yielding zero-bias conductance

$$
G=\bmod \left(n_{\sigma}, 2\right) \frac{2 e^{2}}{h}
$$

The even-odd effect encoded in Eq. (31) represents a "smoking gun" electrical transport signature of bulk Ising non-Abelian anyons. We stress that one can, at least in principle, toggle between the two quantized conductances by locally perturbing the spin liquid far from any electrically active circuit components. Trivial origins for such exotic behavior would appear to require almost divine intervention. At present, however, it remains unclear how to feasibly manipulate Ising anyons so as to probe the evenodd effect in a systematic experiment. A worthwhile preliminary study could instead rely on thermal fluctuations and/or noise to stochastically drag Ising anyons on and off of the gapless spin-liquid edge (for related studies in a quantum-Hall context, see, e.g., Refs. [117-120]). Such processes would change $n_{\sigma}$ as a function of time, leading to telegraph noise with the conductance switching between $G=0$ and $\left(2 e^{2} / h\right)$ as sketched in Fig. 9(c) [121].

The circuits in Figs. 9(a) and 9(b) can be viewed as cousins of " $\mathbb{Z}_{2}$ interferometers" designed to electrically probe physical Majorana fermions in proximitized topological-insulator surfaces $[26,27]$. In that context, the conductance exhibits an analogous even-odd effect, but in the number of superconducting $(h / 2 e)$ vortices threaded through the device. Similar to Sec. V, fermion condensation allows us to adapt such techniques developed for exotic superconductors to probe non-Abelian quasiparticles in a Mott insulator. 
As a technical aside, above we envision creating Fig. 9(b) by dragging an Ising anyon to the gapless boundary of the spin liquid. But if this Ising anyon resides some "small" distance from the edge [see enlargement in Fig. 9(b)], how can one quantify whether the fragmented physical and emergent Majorana fermions encircle only the bulk Ising anyon (corresponding to $n_{\sigma}=1$ ), or also the Ising anyon near the boundary (corresponding to $n_{\sigma}=2$ )? Following Ref. [116], this question can be addressed using a minimal model in which the gapless edge hybridizes with the Majorana zero mode $\gamma$ localized to the adjacent Ising anyon. The Hamiltonian reads

$$
\mathcal{H}=\int_{x}\left[-i v \gamma_{R} \partial_{x} \gamma_{R}+i \lambda \gamma_{R} \gamma \delta(x)\right]
$$

where $\gamma_{R}$ describes the emergent gapless Majorana fermion, and $\lambda$ is the coupling strength to $\gamma$, assumed to reside at position $x=0$. Note that $\lambda^{2} / v$ defines an energy scale for the hybridization.

Reference [116] showed that an incident Majorana fermion with energy $E$ acquires a phase shift

$$
e^{i \phi(E)}=\frac{2 E+i \lambda^{2} / v}{2 E-i \lambda^{2} / v}
$$

due to the $\lambda$ coupling. The "high-" and "low-" energy limits of this result can be captured intuitively as follows. At incident energies $E \gg \lambda^{2} / v$, the gapless edge and the adjacent Ising anyon essentially decouple; in this "highenergy" regime the physical and Majorana fermions should be viewed as encircling both Ising anyons in Fig. 9(b). No additional $\pi$ phase shift arises, though finite, nonuniversal conductance generically emerges due to Andreev processes (which freeze out only at low energies). At $E \ll \lambda^{2} / v$, one can project onto Hamiltonian eigenstates by sending $\gamma_{R}(x) \rightarrow \operatorname{sgn}(x) \tilde{\gamma}_{R}(x)$, where $\tilde{\gamma}_{R}(x)$ is a slowly varying chiral Majorana fermion. In terms of $\tilde{\gamma}_{R}$, the $\lambda$ term in Eq. (32) disappears due to the sign change introduced above, so that $\mathcal{H} \rightarrow \int_{x}\left(-i v \tilde{\gamma}_{R} \partial_{x} \tilde{\gamma}_{R}\right)$. In this precise sense, the adjacent Ising anyon has been absorbed by the gapless edge-its only trace is the $\pi$ phase shift inherent in the definition of $\tilde{\gamma}_{R}$. Hence, the physical and emergent Majorana fermions should now be viewed as encircling only the bulk Ising anyon in Fig. 9(b). Our transport analysis focuses on the asymptotic low-energy limit, where the latter scenario prevails. Both extremes captured above are consistent with the general formula in Eq. (33).

\section{INTERFEROMETRIC DETECTION OF NEUTRAL FERMIONS, ISING ANYONS, AND NON-ABELIAN STATISTICS}

The circuit introduced in Sec. VI reveals bulk Ising anyons but is oblivious to the presence of bulk neutral fermions. This dichotomy arises because an emergent fermion living at the boundary acquires a statistical minus sign upon encircling an Ising anyon, in turn influencing the electrical conductance, whereas encircling a neutral fermion yields a trivial statistical phase. Here we study an interferometer that enables emergent fermions injected from a lead (with the aid of fermion condensation) to splinter into unpaired Ising anyons-which exhibit nontrivial braiding statistics with both bulk Ising anyons and neutral fermions, leading to conductance signatures of both quasiparticle types.

The device we consider appears in Fig. 10(a) and can be viewed as Fig. 9(a) with a constriction. At the constriction, the upper and lower spin-liquid edges couple via a Hamiltonian

(a)

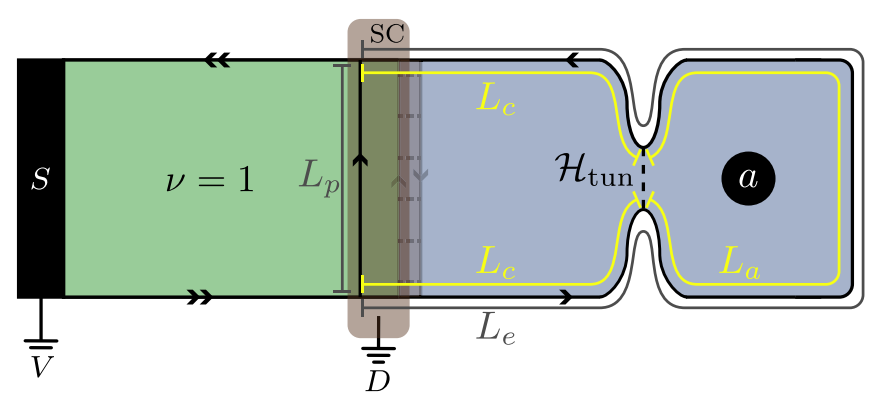

(b)

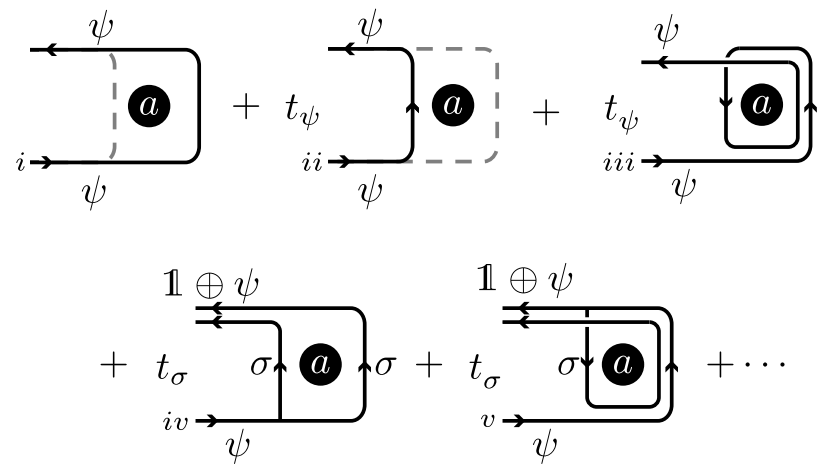

FIG. 10. (a) Interferometer that electrically detects both bulk Ising anyons and bulk emergent fermions. The geometry can be viewed as Fig. 9 with a constriction in the spin liquid, governed by $\mathcal{H}_{\text {tun }}$ in Eq. (34). At the constriction, incident emergent Majorana fermions can either tunnel across ( $t_{\psi}$ process) or fractionalize into a pair of Ising anyons ( $t_{\sigma}$ process) - one hopping across and the other encircling a bulk quasiparticle of type $a=I, \psi$, or $\sigma$. Nontrivial braiding statistics among the anyons in the spin liquid yields $a$-dependent electrical conductances [Eqs. (57) and (59)] that enable readout of the quasiparticle type. Most notably, non-Abelian statistics between Ising anyons vanquishes first-order conductance corrections from $t_{\sigma}$ events. (b) Illustration of the five paths taken by incident emergent fermions up to first order in $\mathcal{H}_{\text {tun }}$. For details, see Secs. VII A and VII B. The ellipsis denotes higher-order contributions not included here. 


$$
\mathcal{H}_{\text {tun }}=e^{-i \pi h_{\sigma}} t_{\sigma} \sigma\left(x_{2}\right) \sigma\left(x_{1}\right)+e^{-i \pi h_{\psi}} t_{\psi} \gamma\left(x_{2}\right) \gamma\left(x_{1}\right)
$$

with $\sigma(x)$ the Ising conformal field theory (CFT) field and $t_{\sigma}, t_{\psi} \geq 0$ real couplings. The $t_{\sigma}$ and $t_{\psi}$ terms respectively shuttle Ising anyons and emergent fermions between positions $x_{1}$ on the lower edge and $x_{2}$ on the upper edge. (For a detailed discussion of the $t_{\sigma}$ term, see Ref. [122].) The phase factors in Eq. (34) involve the topological spin $h_{\sigma}=1 / 16$ of an Ising anyon and $h_{\psi}=1 / 2$ of a fermion, and are required for Hermiticity. Note that $e^{-i \pi h_{\psi}}=-i$ represents the usual imaginary coefficient accompanying a Majorana-fermion bilinear in the Hamiltonian; we employ this form simply to parallel the $t_{\sigma}$ term.

Ising-anyon tunneling constitutes a relevant perturbation to the fixed point describing decoupled edges, and in the asymptotic low-energy limit effectively chops the spin liquid in two at the constriction [122]. Fermion tunneling, by contrast, is marginal. Throughout, we work in a regime - to be quantified below-where both tunneling terms can be regarded as weak. Incident fermions at the lower edge then bypass the constriction and take "the long way around" with nearly unit probability, enabling a perturbative treatment of $\mathcal{H}_{\text {tun }}$.

Let us then examine Fig. 10(a) at temperatures $T \rightarrow 0$ and finite bias voltages $V$ below the gap scale for the fermion condensate. We are interested in the conductance $G(V)$ when a quasiparticle of type $a=\mathbb{1}, \sigma$, or $\psi$ resides in the right half of the interferometer. Figure 10(b) sketches the five contributing processes up to first order in $t_{\psi}$ and $t_{\sigma}$. Path (i) corresponds to the dominant process whereby the incident edge emergent fermion bypasses the constriction and goes around quasiparticle $a$ (as necessarily occurs in Fig. 9). In path (ii), the incident fermion shortcuts across the constriction via the fermion-tunneling term $t_{\psi}$. In path (iii), the fermion travels to the upper side of the constriction before similarly tunneling via $t_{\psi}$. Paths (iv) and (v) invoke Ising-anyon tunneling $t_{\sigma}$. In (iv), the incident fermion travels to the lower end of the constriction, then splinters into a pair of Ising anyons that recombine at the upper edge into either a trivial particle or an emergent fermion depending on $a$. And in (v), the incident fermion travels to the upper side of the constriction before similarly splintering into Ising anyons. In what follows, we examine these processes within a phenomenological treatment that we eventually connect to more formal analyses given in Appendixes C and D (see also the analyses of related interferometers, e.g., in Refs. [123-125]). We start with the case $a=\mathbb{1}$ or $\psi$ and then consider $a=\sigma$.

\section{A. Interferometer with $a=\mathbb{1}$ or $\psi$}

When $a=\mathbb{1}$ or $\psi$, the splintered Ising anyons from paths (iv) and (v) of Fig. 10(b) necessarily recombine into an outgoing emergent fermion at the upper edge (braiding $\sigma$ around either $\mathbb{1}$ or $\psi$ preserves the Ising anyons' fusion channel). Thus, in all five paths, emergent Majorana fermions incident from below necessarily exit the interferometer as fermions. The conductance simply follows from the phase accumulated en route. We now separately examine each path from Fig. 10(b).

Path (i). Consider an emergent Majorana fermion with momentum $k_{e}$ that travels a distance $L_{e}$ the long way around the interferometer. The associated quantum amplitude reads

$$
A_{i}=e^{i k_{e} L_{e}},
$$

which is simply the phase acquired by the fermion.

For the remaining cases, it is useful to express the amplitude for path $(p)$ as $A_{p}=w_{p} e^{i \phi_{p}}$; here, $w_{p}$ encodes local physics at the constriction and $e^{i \phi_{p}}$ captures the phase accumulated due to propagation along the boundary. Note that $w_{i i}$ and $w_{i i i}$ are proportional to $t_{\psi}$ while $w_{i v}$ and $w_{v}$ are proportional to $t_{\sigma}$.

Path (ii). If the fermion propagates to the lower end of the constriction and then tunnels across, the amplitude is

$$
A_{i i}=w_{i i} e^{i k_{e}\left(L_{e}-L_{a}\right)},
$$

where $L_{a}$ is the perimeter of the region enclosing $a$ as sketched in Fig. 10(a).

Path (iii). Let $L_{c}$ be the distance between the constriction and either end of the fermion condensate [see Fig. 10(a)]. The amplitude for path (iii) can then be written

$$
A_{i i i}=w_{i i i} e^{i k_{e}\left(L_{c}+L_{a}\right)} e^{i k_{e}\left(L_{a}+L_{c}\right)}
$$

The first exponential represents the phase acquired upon traveling to the upper part of the constriction, and the second is the phase acquired by the fermion after tunneling across the constriction and returning to the fermion condensate. Noting that $L_{e}=2 L_{c}+L_{a}$, we can simplify Eq. (37) to

$$
A_{i i i}=w_{i i i} e^{i k_{e}\left(L_{e}+L_{a}\right)} .
$$

Path (iv). When the incident Majorana fermion splinters in path (iv), its momentum $k_{e}$ can partition among the resulting pair of Ising anyons in various ways that are compatible with energy conservation [126]. Suppose for now that the Ising anyon tunneling across the constriction carries momentum $k_{1}$, while the Ising anyon that takes the long way around carries $k_{2}=k_{e}-k_{1}$. The amplitude for this event is

$$
\begin{aligned}
A_{i v}\left(k_{1}\right) & =w_{i v}\left(k_{e}, k_{1}\right) e^{i k_{e} L_{c}} e^{i k_{1} L_{c}} e^{i k_{2}\left(L_{a}+L_{c}\right)}(-1)^{n_{\psi}} \\
& =w_{i v}\left(k_{e}, k_{1}\right) e^{i k_{e} L_{e}} e^{-i k_{1} L_{a}}(-1)^{n_{\psi}} .
\end{aligned}
$$

Here, the factor $w_{i v}$ generically depends on both $k_{e}$ and $k_{1}$ as a consequence of the relevance of the Ising-anyon 
tunneling term. The first exponential on the top line is the phase acquired by the fermion as it travels to the constriction; the second is the phase acquired by the Ising anyon that tunnels across the constriction and travels to the upper end of the fermion condensate, and the third is the phase acquired by the Ising anyon that travels the long way around. In the last factor, $n_{\psi}$ is the number of bulk neutral fermions (mod 2) enclosed in the right half of the interferometer, i.e., $n_{\psi}=0$ if $a=\mathbb{1}$, while $n_{\psi}=1$ if $a=\psi$. The all-important additional $\pi$ phase that arises when $n_{\psi}=1$ reflects the nontrivial mutual statistics between Ising anyons and neutral fermions, and ultimately allows the interferometer to detect the latter bulk quasiparticle type.

Events corresponding to distinct, physically permissible $k_{1}$ values must be integrated over since the wave function will consist of a weighted sum over all such energy partitionings. In particular, the pair of splintered Ising anyons must both carry positive momentum and energy, yielding the inequality $0 \leq k_{1} \leq k_{e}$ for path (iv).

Path (v). Suppose now that an incident emergent Majorana fermion in path (v) similarly splinters into one Ising anyon carrying momentum $k_{1}$ across the constriction and another that carries momentum $k_{2}=k_{e}-k_{1}$ past the constriction. The corresponding amplitude is

$$
\begin{aligned}
A_{v}\left(k_{1}\right) & =w_{v}\left(k_{e}, k_{1}\right) e^{i k_{e}\left(L_{c}+L_{a}\right)} e^{i k_{1}\left(L_{a}+L_{c}\right)} e^{i k_{2} L_{c}}(-1)^{n_{\psi}} \\
& =w_{v}\left(k_{e}, k_{1}\right) e^{i k_{e} L_{e}} e^{i k_{1} L_{a}}(-1)^{n_{\psi}} .
\end{aligned}
$$

The first three exponentials in the top line respectively denote the phase acquired by the fermion prior to splintering, the Ising anyon that tunnels across the constriction, and the Ising anyon that bypasses the constriction. The $(-1)^{n_{\psi}}$ factor once again reflects the braiding statistics between Ising anyons and neutral fermions.

Physically permissible $k_{1}$ values must be integrated over, as in path (iv), but now the allowed range differs. Indeed, here the Ising anyon that tunnels across the constriction can carry arbitrary positive momentum since the pair of Ising anyons that combines on the upper end of the interferometer always carries total momentum $k_{e}$ regardless of the magnitude of $k_{1}$. For path (v), we thus have the inequality $0 \leq k_{1}<\infty$ (neglecting an ultraviolet momentum cutoff for simplicity).

Upon summing over the five paths, the amplitude describing the arrival of the emergent Majorana fermion at the upper end of the fermion condensate is

$$
\begin{aligned}
A= & A_{i}+A_{i i}+A_{i i i} \\
& +\int_{0}^{k_{e}} d k_{1} A_{i v}\left(k_{1}\right)+\int_{0}^{\infty} d k_{1} A_{v}\left(k_{1}\right) .
\end{aligned}
$$

Inserting the above expressions for $A_{i}$ through $A_{v}$ yields

$$
\begin{aligned}
A= & e^{i k_{e} L_{e}}\left\{1+\left[w_{i i} e^{-i k_{e} L_{a}}+w_{i i i} e^{i k_{e} L_{a}}\right]\right. \\
& +(-1)^{n_{\psi}}\left[\int_{0}^{k_{e}} d k_{1} w_{i v}\left(k_{e}, k_{1}\right) e^{-i k_{1} L_{a}}\right. \\
& \left.\left.+\int_{0}^{\infty} d k_{1} w_{v}\left(k_{e}, k_{1}\right) e^{i k_{1} L_{a}}\right]\right\} .
\end{aligned}
$$

We can further constrain the form of the amplitude using dimensional analysis. Since the scaling dimension of the Majorana fermion $\gamma(x)$ is $1 / 2, t_{\psi}$ has units of energy $\times$ length; similarly, the Ising field $\sigma(x)$ scaling dimension is $1 / 16$, and so $t_{\sigma}$ has units of energy $x$ (length $)^{1 / 8}$. Thus, we can write

$$
\begin{gathered}
w_{i i, i i i}=\left[\frac{t_{\psi}}{v_{e}}\right] \alpha_{i i, i i i}, \\
w_{i v, v}\left(k_{e}, k_{1}\right)=\left[\frac{t_{\sigma}}{v_{e} k_{e}^{7 / 8}}\right] \frac{f_{i v, v}\left(k_{1} / k_{e}\right)}{k_{e}}
\end{gathered}
$$

with $v_{e}$ the emergent-fermion edge-state velocity, $\alpha_{i i}$ and $\alpha_{i i i}$ numerical factors, and $f_{i v}$ and $f_{v}$ dimensionless scaling functions. Notice that the bracketed factors above are dimensionless.

Determining the remaining unspecified quantities in $A$ requires explicit calculations. For the fermion-tunneling paths, Appendix C presents a standard Heisenberg-picture analysis that yields

$$
\alpha_{i i}=-\alpha_{i i i}=-\frac{1}{2}
$$

Equations (43) through (45) then allow us to express the amplitude as

$$
\begin{aligned}
A= & e^{i k_{e} L_{e}}\left[1+i \frac{t_{\psi}}{v_{e}} \sin \left(k_{e} L_{a}\right)\right. \\
& \left.+i(-1)^{n_{\psi}} \frac{2 t_{\sigma} L_{a}^{7 / 8}}{v_{e}} g\left(k_{e} L_{a}\right)\right],
\end{aligned}
$$

where we define

$$
g(u)=\frac{1}{2 i u^{7 / 8}}\left[\int_{0}^{1} d y e^{-i u y} f_{i v}(y)+\int_{0}^{\infty} d y e^{i u y} f_{v}(y)\right] .
$$

Treating the Ising-anyon tunneling paths demands a more sophisticated conformal field theory analysis carried out in Appendix D. There we show that

$$
g(u)=\frac{\pi u}{4}{ }_{1} F_{2}\left(\frac{1}{2} ; 1,2 \mid-\frac{1}{4} u^{2}\right)
$$


(a)

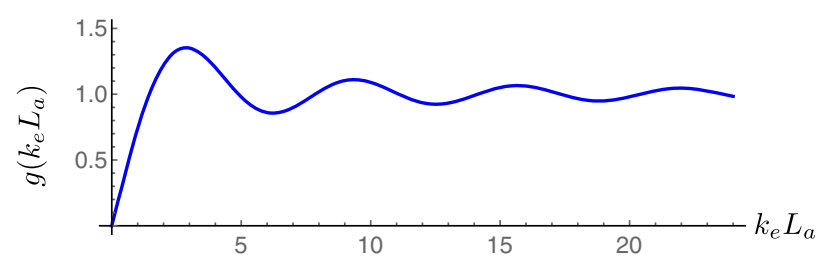

(b)

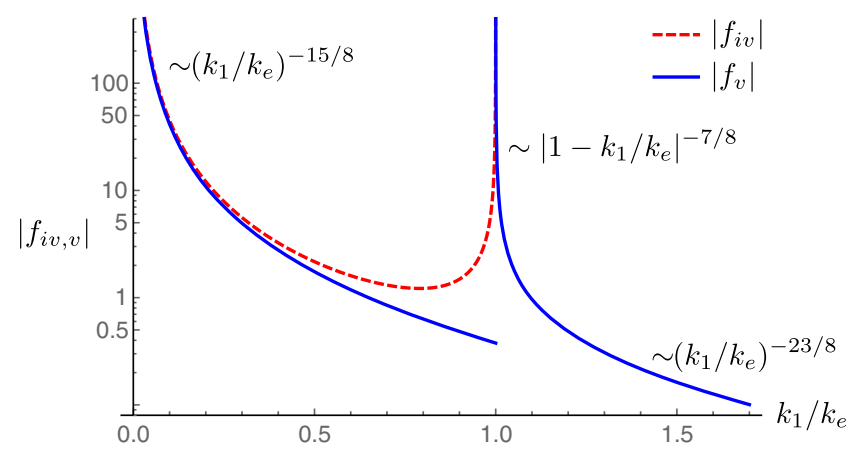

FIG. 11. (a) Plot of $g\left(k_{e} L_{a}\right)$ [Eq. (48)] versus $k_{e} L_{a}$, where $k_{e}$ denotes the incoming emergent-fermion momentum and $L_{a}$ is the length defined in Fig. 10(a). This function determines the $t_{\sigma}$ correction to the fermion transmission amplitude [Eq. (46)], and thus also the conductance [Eq. (57)], for the interferometer in Fig. 10(a) when $a=I$ or $\psi$. (b) Magnitude of the scaling functions $f_{i v}$ (red dashed line) and $f_{v}$ (blue line) obtained by inverting Eq. (47) as carried out in Appendix F. These scaling functions govern energy partitioning associated with Ising-anyon tunneling in paths (iv) and (v) summarized in Fig. 10(b) and discussed in Sec. VII A; recall Eq. (44). In the horizontal axis $k_{1}$ is the momentum carried by an Ising anyon that tunnels across the constriction. The limiting scaling behavior displayed above implies that Ising anyons tunnel primarily carrying momentum $\ll k_{e}$ and secondarily carrying momentum very near $k_{e}$.

with ${ }_{1} F_{2}$ a generalized hypergeometric function. Figure 11(a) plots $g\left(k_{e} L_{a}\right)$ versus $k_{e} L_{a}$. The perturbative regime stipulated earlier requires [128]

$$
\frac{t_{\psi}}{v_{e}} \ll 1 \quad \text { and } \quad \frac{t_{\sigma} L_{a}^{7 / 8}}{v_{e}} \ll 1
$$

so that fermion and Ising-anyon tunneling across the constriction contribute only small corrections to the amplitude in Eq. (46).

To make contact with our phenomenological picture, we can invert Eq. (47) to extract the $f_{i v}\left(k_{1} / k_{e}\right)$ and $f_{v}\left(k_{1} / k_{e}\right)$ scaling functions that quantify the energy partitioning. Appendix F pursues this (nontrivial) exercise; for a summary, see Fig. 11(b). Both scaling functions exhibit a leading divergence at $k_{1} \rightarrow 0$ and a subleading divergence at $k_{1} \rightarrow k_{e}$. It follows that Ising anyons tunnel across the constriction primarily carrying "small" momentum and secondarily carrying momentum near $k_{e}$. More explicitly, $f_{i v, v}\left(k_{1} / k_{e}\right) \sim\left(k_{1} / k_{e}\right)^{-15 / 8}$ at small $k_{1}$; the exponent implies that the $k_{e}$ dependence of the weights defined in Eq. (44) drops out at $k_{1} \rightarrow 0$; i.e., in this regime, the Isinganyon tunneling probability becomes independent of the incident fermion momentum. Furthermore, $f_{i v}\left(k_{1} / k_{e}\right) \sim$ $\left(1-k_{1} / k_{e}\right)^{-7 / 8}$ as $k_{1}$ approaches $k_{e}$ from below and $f_{v}\left(k_{1} / k_{e}\right) \sim\left(k_{1} / k_{e}-1\right)^{-7 / 8}$ as $k_{1}$ approaches $k_{e}$ from above. Notice that $f_{v}\left(k_{1} / k_{e}\right)$ does not diverge as $k_{1}$ approaches $k_{e}$ from below-hence, for path (v), Ising anyons tunnel far more efficiently with momentum slightly larger than $k_{e}$ compared to momentum just below $k_{e}$.

It is instructive to examine some limits of the function $g\left(k_{e} L_{a}\right)$. At small arguments, one finds

$$
g\left(k_{e} L_{a} \ll 1\right) \approx \frac{\pi}{4} k_{e} L_{a} ;
$$

i.e., the amplitude correction from Ising-anyon tunneling vanishes linearly with the fermion momentum. In this limit of our perturbative analysis, one can use an operator product expansion (OPE) to fuse the Ising CFT fields in Eq. (34), yielding

$$
e^{-i \pi h_{\sigma}} t_{\sigma} \sigma\left(x_{2}\right) \sigma\left(x_{1}\right) \rightarrow \text { const } \times t_{\sigma} L_{a}^{15 / 8} \gamma \partial_{x} \gamma .
$$

The term on the right side (which is a descendent of the identity) is irrelevant, which explains the linear vanishing of the amplitude correction as $k_{e} L_{a} \rightarrow 0$.

At $k_{e} L_{a} \gg 1$, one instead finds

$$
g\left(k_{e} L_{a} \gg 1\right) \approx 1-\frac{\cos \left(k_{e} L_{a}\right)}{k_{e} L_{a}} .
$$

Contrary to the purely oscillatory amplitude correction from fermion tunneling, the amplitude correction from Ising-anyon tunneling thus tends to an $L_{a}$-dependent constant as $k_{e} L_{a} \rightarrow \infty$, with subdominant oscillations that decay with a $1 /\left(k_{e} L_{a}\right)$ prefactor. The former feature reflects the propensity of Ising anyons to tunnel across the constriction with vanishingly small momentum. Oscillations in the latter piece come from Ising anyons that tunnel with momentum near $k_{e}$, while the decay arises because of the finite spread in the allowed momentum carried.

The tunneling Hamiltonian invoked in Eq. (34) could of course be amended in various ways, e.g., by allowing fermions and Ising anyons to tunnel over a finite range of positions between the upper and lower sides of the constriction (as opposed to discrete points $x_{1,2}$ ) or by adding derivatives that effectively make the tunnel couplings momentum dependent. It is thus important to address which features of the amplitude correction in Eq. (46) are generic. The linear vanishing of both the $t_{\psi}$ and $t_{\sigma}$ corrections with $k_{e}$ [cf. Eq. (50)] is certainly universal (though the prefactor of course is not). At $k_{e} \rightarrow 0$, one can 
exploit an OPE similar to Eq. (51) to reduce arbitrary fermion-tunneling and Ising-anyon tunneling terms to irrelevant descendants of the identity. The leading irrelevant term is $\gamma \partial_{x} \gamma$, and the derivative ensures amplitude corrections $\propto k_{e}$ as $k_{e} \rightarrow 0$.

We further expect that the exponents governing the power-law divergences in the $f_{i v, v}$ scaling functions shown in Fig. 11(b) are universal. At $k_{e} L_{a} \gg 1$, these divergences determine the leading and subleading $k_{e} L_{a}$ dependence of the $t_{\sigma}$ correction specified by Eq. (52) - which would then also be universal. Note, however, that the prefactors of the two terms in Eq. (52) will depend on details of the tunneling Hamiltonian. The generic saturation of the $t_{\sigma}$ correction to an $L_{a}$-dependent constant at $k_{e} L_{a} \rightarrow \infty$ admits an intuitive explanation: Energy partitioning invariably suppresses oscillations as $k_{e} L_{a}$ increases, whereas processes for which Ising anyons tunnel across the constriction carrying vanishingly small momentum naturally leave a $k_{e}$-independent correction. [Again, the Ising-anyon tunneling weights in Eq. (44) do not depend on the incident fermion momentum in the $k_{1} \rightarrow 0$ limit.]

We are now ready to extract the conductance. The net phase acquired by an incident emergent Majorana fermion is given by

$$
e^{i \phi_{\text {emergent }}}=\frac{A}{|A|} \text {. }
$$

Moreover, the phase acquired by a physical Majorana fermion with momentum $k_{p}$ that travels the length $L_{p}$ of the fermion condensate is

$$
e^{i \phi_{\text {physical }}}=e^{i k_{p} L_{p}}
$$

yielding a phase difference

$$
\begin{aligned}
& \delta \phi_{a=1, \psi}\left(k_{e}, k_{p}\right) \\
& =k_{p} L_{p}-k_{e} L_{e}-\frac{t_{\psi}}{v_{e}} \sin \left(k_{e} L_{a}\right)-(-1)^{n_{\psi}} \frac{2 t_{\sigma} L_{a}^{7 / 8}}{v_{e}} g\left(k_{e} L_{a}\right)
\end{aligned}
$$

to first order in $t_{\psi}, t_{\sigma}$; cf. Eq. (28). Suppose next that an incident electron injected from the lead into the lower $\nu=1$ edge of Fig. 10(a) carries energy $E$. The Majorana-fermion momenta are then $k_{e}=E / v_{e}$ and $k_{p}=E / v_{p}$, where $v_{p}$ is the physical Majorana fermion's edge velocity. As reviewed in Appendix G, the conductance at bias voltage $V$ is

$G_{a=1, \psi}(V)=\frac{e^{2}}{h}\left\{1-\cos \left[\delta \phi_{a=1, \psi}\left(\frac{e V}{v_{e}}, \frac{e V}{v_{p}}\right)\right]\right\}$.

Finally, expanding in $t_{\psi}$ and $t_{\sigma}$ yields

$$
\begin{aligned}
G_{a=1, \psi}(V) & \approx \\
\approx & \frac{e^{2}}{h}\left\{1-\cos \left[e V\left(\frac{L_{p}}{v_{p}}-\frac{L_{e}}{v_{e}}\right)\right]\right. \\
& -\frac{t_{\psi}}{2 v_{e}} \cos \left[e V\left(\frac{L_{p}}{v_{p}}-\frac{L_{e}+L_{a}}{v_{e}}\right)\right] \\
& +\frac{t_{\psi}}{2 v_{e}} \cos \left[e V\left(\frac{L_{p}}{v_{p}}-\frac{L_{e}-L_{a}}{v_{e}}\right)\right] \\
& \left.-(-1)^{n_{\psi}} \frac{2 t_{\sigma} L_{a}^{7 / 8}}{v_{e}} g\left(e V \frac{L_{a}}{v_{e}}\right) \sin \left[e V\left(\frac{L_{p}}{v_{p}}-\frac{L_{e}}{v_{e}}\right)\right]\right\} .
\end{aligned}
$$

In the first line, the oscillatory voltage dependence reflects the periodic revival and destruction of Andreev processes as the phase difference accumulated by the physical and emergent Majorana fermions varies in path (i). The next two lines encode corrections from fermion tunneling across the constriction; hence, the dependence on the shifted path lengths $L_{e} \pm L_{a}$. And by far most importantly, the correction from Ising-anyon tunneling in the last line reveals the presence of individual emergent fermions by virtue of the $n_{\psi}$ dependence.

\section{B. Interferometer with $a=\sigma$}

Suppose now that $a=\sigma$ (which turns out to be far simpler to analyze compared to the $a=\mathbb{1}$ or $\psi$ cases). We assume that the bulk Ising anyon's "partner" has been dragged to an adjacent gapless part of the edge in the right half of the interferometer. Path (i) acquires an additional $\pi$ phase relative to Eq. (35) due to the nontrivial mutual statistics between fermions and Ising anyons. By contrast, the phases from paths (ii) and (iii) - wherein the edge fermion encircles the bulk Ising anyon an even number of times - conform exactly to Eqs. (36) and (38), respectively [129]. In paths (iv) and (v), the incident emergent fermion splinters into two Ising anyons at the constriction, one of which now encircles a bulk Ising anyon. This braiding process changes the fusion channel for the splintered edge Ising anyons from $\psi$ to $\mathbb{1}$. More physically, in paths (iv) and (v) the incident emergent fermion exits the interferometer as a trivial boson, and hence, these paths no longer contribute to the electrical conductance.

The amplitude from Eq. (46) accordingly becomes

$$
A=e^{i k_{e} L_{e}}\left[-1+i \frac{t_{\psi}}{v_{e}} \sin \left(k_{e} L_{a}\right)\right] .
$$

Following precisely the same steps outlined in the preceding section, one obtains a conductance 


$$
\begin{aligned}
G_{a=\sigma}(V) \approx & \frac{e^{2}}{h}\left\{1+\cos \left[e V\left(\frac{L_{p}}{v_{p}}-\frac{L_{e}}{v_{e}}\right)\right]\right. \\
& -\frac{t_{\psi}}{2 v_{e}} \cos \left[e V\left(\frac{L_{p}}{v_{p}}-\frac{L_{e}+L_{a}}{v_{e}}\right)\right] \\
& \left.+\frac{t_{\psi}}{2 v_{e}} \cos \left[e V\left(\frac{L_{p}}{v_{p}}-\frac{L_{e}-L_{a}}{v_{e}}\right)\right]\right\} .
\end{aligned}
$$

In the limit $t_{\sigma}=t_{\psi}=0$, the interferometer effectively reduces to the setup in Figs. 9(a) and 9(b) that allow electrical detection of Ising anyons. Indeed, Eqs. (57) and (59), respectively, yield $G=0$ and $G=\left(2 e^{2} / h\right)$ at $V \rightarrow 0$, in agreement with the analysis from Sec. VI. Allowing quasiparticle tunneling across the constriction additionally reveals the non-Abelian statistics of Ising anyons as manifested by the disappearance of the oscillatory $t_{\sigma}$ correction in Eq. (57) when a bulk Ising anyon sits in the interferometer loop. Qualitatively similar physics appears in quantum-Hall interferometers introduced in Refs. [9-11].

\section{DISCUSSION}

A growing body of evidence supports the realization of a non-Abelian spin-liquid phase in the Kitaev material $\alpha-\mathrm{RuCl}_{3}$ [50,54-61,64]. This remarkable development strongly motivates proposals for probing individual fractionalized excitations in honeycomb Kitaev materials, as required for eventual topological-quantum-computing applications. The Mott-insulating nature of the host platform renders the problem both interesting and nontrivial. We introduce a strategy based, counterintuitively, on universal low-voltage electrical transport in novel circuits designed to perfectly convert electrons into emergent Majorana fermions born in the spin liquid, and vice versa. Similar techniques can be adapted to any bosonic topologically ordered phase hosting gapless emergent-fermion edge states.

Perfect physical-fermion $\leftrightarrow$ emergent-fermion conversion transpires when the non-Abelian spin liquid's chiral Majorana edge state gaps out with a counterpropagating 1D electronic channel-forming a fermion condensate. Throughout this paper, we consider proximitized $\nu=1$ integer quantum-Hall systems as the source of 1D electrons participating in fermion condensation. We adopt this choice due to the wide availability of $\nu=1$ states and for convenience in defining electrical transport quantities. As a possible variation, one could replace the quantumHall system with a spinless 2D $p+i p$ superconductor, which supports "half" of a $\nu=1$ edge state and thus admits a fully gapped interface with the spin liquid. Alternatively, one could employ proximitized 1D wires instead of 2D topological phases at the expense of introducing additional electronic channels. For illustrations, see Fig. 12. Exploring (a)

(b)
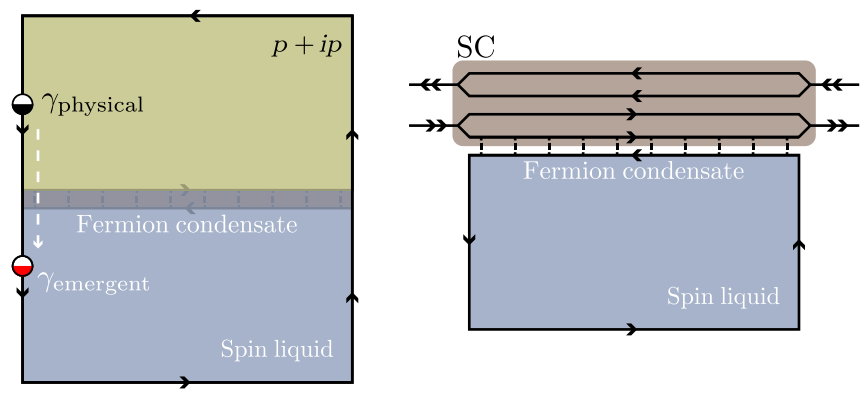

FIG. 12. Variations on Fig. 7 in which the $\nu=1$ quantum-Hall system is replaced by (a) a 2D spinless $p+i p$ superconductor and (b) a proximitized 1D wire. Both alternatives also enable physical-fermion $\leftrightarrow$ emergent-fermion conversion via fermion condensation. In (a), the physical chiral Majorana edge state of a spinless 2D $p+i p$ superconductor gaps out with the spin liquid's emergent chiral Majorana edge mode-yielding a fully gapped interface between the two subsystems. Physical Majorana fermions from the $p+i p$ superconductor thus invariably enter the spin liquid as emergent Majorana fermions. In (b), right- and leftmoving electron channels (double arrows) separate into Majorana modes beneath the proximitizing superconductor. Fermion condensation arises when one of those Majorana modes gaps out with the spin liquid's edge state. (Among the three remaining modes beneath the superconductor, only one must be gapless.)

transport characteristics of circuits employing such variations would certainly be worthwhile.

Moreover, developing a detailed microscopic understanding of the interaction mediating fermion condensation [recall the $\kappa$ term from Eq. (17)] remains an important open problem even for our quantum-Hall-based setups. We anticipate that spin-spin interactions between the quantum-Hall edge and spin-liquid edge (mediated, for instance, by an interplay between Coulomb repulsion and electron tunneling between the subsystems) constitute one natural microscopic mechanism that contributes to $\kappa$. In Sec. III A, we show on symmetry grounds that microscopic spin operators at the Kitaev-material boundary project onto the kinetic energy operator in the chiral Majorana edge theory. We expect that spin operators at the quantum-Hall boundary similarly project onto the kinetic energy for the proximitized quantum-Hall edge - in turn yielding the desired $\kappa$ term. Working out detailed dependences on factors such as Zeeman fields, structure and sign of the spin-spin interaction, and spin-orbit effects-and how they impact the all-important sign of $\kappa$-would be very interesting.

In Sec. V, we see that quantum-Hall-Kitaev-materialquantum-Hall circuits (Fig. 8) reveal the spin liquid's emergent chiral Majorana edge state via quantized zerobias charge conductance. This purely electrical fingerprint complements the well-known quantized thermal Hall signature of a Majorana edge mode [34,60,64], and relies critically on the fermion condensation that underlies our 
anyon-detection schemes. Section VI explores a somewhat simpler quantum-Hall-Kitaev-material device (Fig. 9) designed to electrically detect bulk Ising non-Abelian anyons. In particular, the circuit admits zero-bias conductance quantized at either $G=0$ or $G=2 e^{2} / h$ depending on whether an even or odd number of Ising anyons resides in the bulk. This striking even-odd effect reflects the nontrivial mutual braiding statistics between Ising anyons and emergent fermions. Taking the same circuit and adding a constriction within the spin liquid leads to the interferometer that we explore in Sec. VII (Fig. 10). At the constriction, an emergent fermion can splinter into a pair of Ising anyons - one taking a shortcut across the pinch and the other continuing along the spin-liquid edge. The electrical conductance is then sensitive to the presence of bulk Ising anyons and bulk emergent fermions since Ising anyons exhibit nontrivial braiding statistics upon encircling either quasiparticle type. Notably, non-Abelian braiding statistics is manifested as a vanishing of certain conductance corrections when an odd number of bulk Ising anyons sits in the interferometer [cf. Eqs. (57) and (59)]similar to the physics encountered in fractional quantumHall architectures [9-11].

Analyzing circuits with realistic imperfections poses another worthwhile direction for future investigation. For instance, the superconductors in practice will likely contain low-energy degrees of freedom due to disorder and/or vortices. Electrons from the $\nu=1$ edge could directly hop onto these low-lying modes without encountering the fermion condensate or Andreev reflecting, thereby providing a parallel conduction channel that modifies the conductances predicted in this paper. Thermally excited quasiparticles in the spin liquid can also produce unwanted errors, e.g., due to processes wherein an edge emergent fermion splinters into Ising anyons that enclose a thermally excited bulk quasiparticle residing near the boundary. For our purposes, these corrections must be sufficiently small that (i) a sharp contrast remains between the nontrivial and control circuits in Fig. 8, and (ii) the quasiparticle-dependent conductances predicted for Figs. 9 and 10 remain discernible.

For initial anyon-detection experiments, one could rely on nature to thermally cycle between various bulk quasiparticle configurations-leading to telegraph noise wherein the conductance randomly cycles among the predicted values as a function of time [see, e.g., Fig. 9(c)]. Deterministic, realtime anyon control is nevertheless clearly desirable. To this end, it is essential to develop practical means of trapping Ising anyons and emergent fermions in the spin liquid. We anticipate that this subtle energetics issue can be profitably addressed by studying the Kitaev honeycomb model supplemented by generic symmetry-allowed perturbations. Finally, developing complementary methods of detecting individual bulk anyons that mitigate the experimental requirements of our scheme poses a key challenge. We hope that this work helps stimulate such efforts with the ultimate aim of crafting a realistic road map toward topological quantum computation with Kitaev materials.

\section{ACKNOWLEDGMENTS}

It is a pleasure to thank Chao-Ming Jian, Stevan NadjPerge, Achim Rosch, Ady Stern, and especially Paul Fendley for stimulating conversations. This work is supported by a postdoctoral fellowship from the Gordon and Betty Moore Foundation, under the EPiQS initiative, Grant No. GBMF4304; the Army Research Office under Grant No. W911NF-17-1-0323; the National Science Foundation through Grants No. DMR-1723367 (J. A.) and No. DMR1848336 (R.M.); the Caltech Institute for Quantum Information and Matter, a NSF Physics Frontiers Center with support of the Gordon and Betty Moore Foundation through Grant No. GBMF1250; the Walter Burke Institute for Theoretical Physics at Caltech, and the Gordon and Betty Moore Foundation's EPiQS Initiative, Grant No. GBMF8682 to J. A. B. M. H. acknowledges support from the Department of Energy under the Early Career program (Grant No. DE-SC0018115). D. M. acknowledges support from the Gordon and Betty Moore Foundation EPiQS Initiative, Grant No. GBMF9069. Finally, we acknowledge the 2018 Erice Conference on Majorana Fermions and Topological Materials Science, where this work was initiated.

\section{APPENDIX A: VARIATIONAL ANALYSIS OF INTERACTING MAJORANA FERMIONS}

\section{Continuum model}

Here we employ a variational approach to study the interacting continuum Hamiltonian $\mathcal{H}_{0}+\delta \mathcal{H}$ defined in Eqs. (16) and (17). Specifically, we view the ground state of the free-fermion Hamiltonian $\mathcal{H}_{\mathrm{MF}}$ from Eq. (18) as a trial wave function for the interacting problem, treating the mass $m$ as a variational parameter. Note that sending $\gamma_{R} \rightarrow-\gamma_{R}$ leaves the interacting Hamiltonian invariant but sends $m \rightarrow-m$. Without loss of generality, we therefore consider trial wave functions with $m \geq 0$ below.

The analysis is most conveniently carried out in terms of momentum-space Majorana fermions defined with Fouriertransform conventions

$$
\begin{aligned}
& \gamma_{A}(x)=\frac{1}{\sqrt{2}} \int \frac{d k}{2 \pi} e^{i k x} \gamma_{A, k}, \\
& \gamma_{A, k}=\sqrt{2} \int d x e^{-i k x} \gamma_{A}(x)
\end{aligned}
$$

for $A=L$ or $R$. The corresponding commutation relations are given by 


$$
\begin{gathered}
\left\{\gamma_{A}(x), \gamma_{B}(y)\right\}=\frac{1}{2} \delta_{A B} \delta(x-y), \\
\left\{\gamma_{A, k}, \gamma_{B, q}\right\}=2 \pi \delta_{A B} \delta(k+q) .
\end{gathered}
$$

Upon passing to momentum space, the free-fermion Hamiltonian $\mathcal{H}_{\mathrm{MF}}$ can be readily diagonalized, yielding single-particle excitation energies $E(k)=\sqrt{(v k)^{2}+(m / 2)^{2}}$ and correlation functions

$$
\begin{gathered}
\left\langle\gamma_{R, k} \gamma_{R, q}\right\rangle=2 \pi \delta(k+q) \frac{1}{2}\left[1+\frac{v k}{E(k)}\right], \\
\left\langle\gamma_{L, k} \gamma_{L, q}\right\rangle=2 \pi \delta(k+q) \frac{1}{2}\left[1-\frac{v k}{E(k)}\right], \\
\left\langle\gamma_{R, k} \gamma_{L, q}\right\rangle=2 \pi \delta(k+q) \frac{i}{4} \frac{m}{E(k)} .
\end{gathered}
$$

Here and in the remainder of this Appendix, expectation values are taken with respect to the ground state of $\mathcal{H}_{\mathrm{MF}}$.

Equations (A5) through (A7) allow one to efficiently evaluate the trial energy density $\mathcal{E}_{\text {trial }} \equiv\left\langle\mathcal{H}_{0}+\delta \mathcal{H}\right\rangle / L(L$ is the length of the interface). The results are conveniently expressed in terms of integrals

$$
I_{\alpha}=\int_{-2 v \Lambda / m}^{2 v \Lambda / m} d u \frac{u^{\alpha}}{\sqrt{1+u^{2}}}
$$

where $\Lambda$ is a momentum cutoff for the interacting Majorana fermions. The kinetic energy piece reads

$$
\frac{\left\langle\mathcal{H}_{0}\right\rangle}{L}=-\frac{m^{2}}{16 \pi v} I_{2}
$$

For the interactions, we first write

$$
\frac{\langle\delta \mathcal{H}\rangle}{L}=\frac{\kappa}{4} \int_{q_{1}, q_{2}, q_{3}, q_{4}} q_{2} q_{4}\left\langle\gamma_{R, q_{1}} \gamma_{R, q_{2}} \gamma_{L, q_{3}} \gamma_{L, q_{4}}\right\rangle
$$

and then evaluate the expectation value in the integrand using Wick's theorem:

$$
\begin{aligned}
\left\langle\gamma_{R, q_{1}} \gamma_{R, q_{2}} \gamma_{L, q_{3}} \gamma_{L, q_{4}}\right\rangle= & \left\langle\gamma_{R, q_{1}} \gamma_{R, q_{2}}\right\rangle\left\langle\gamma_{L, q_{3}} \gamma_{L, q_{4}}\right\rangle \\
& -\left\langle\gamma_{R, q_{1}} \gamma_{L, q_{3}}\right\rangle\left\langle\gamma_{R, q_{2}} \gamma_{L, q_{4}}\right\rangle \\
& +\left\langle\gamma_{R, q_{1}} \gamma_{L, q_{4}}\right\rangle\left\langle\gamma_{R, q_{2}} \gamma_{L, q_{3}}\right\rangle .
\end{aligned}
$$

Some algebra yields

$$
\frac{\langle\delta \mathcal{H}\rangle}{L}=-\frac{\kappa m^{4}}{1024 \pi^{2} v^{4}}\left(I_{2}^{2}+I_{0} I_{2}\right) .
$$

Summing the contributions above gives our trial energy density, (a)

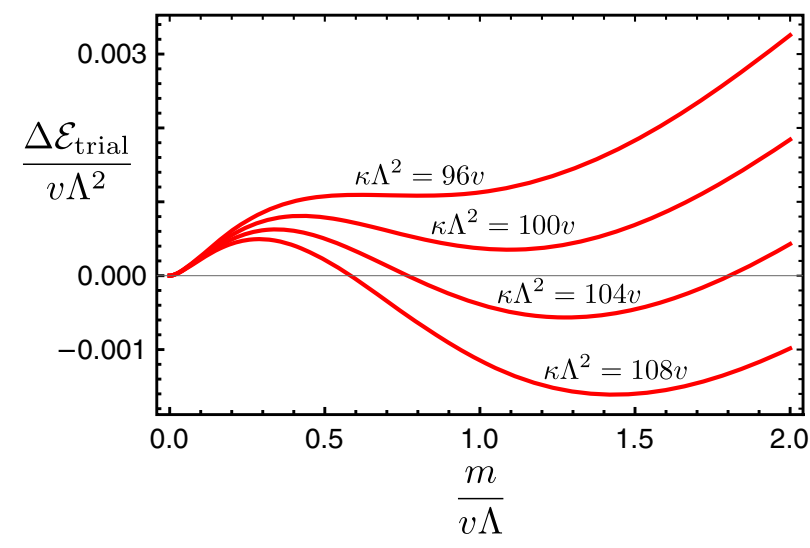

(b)

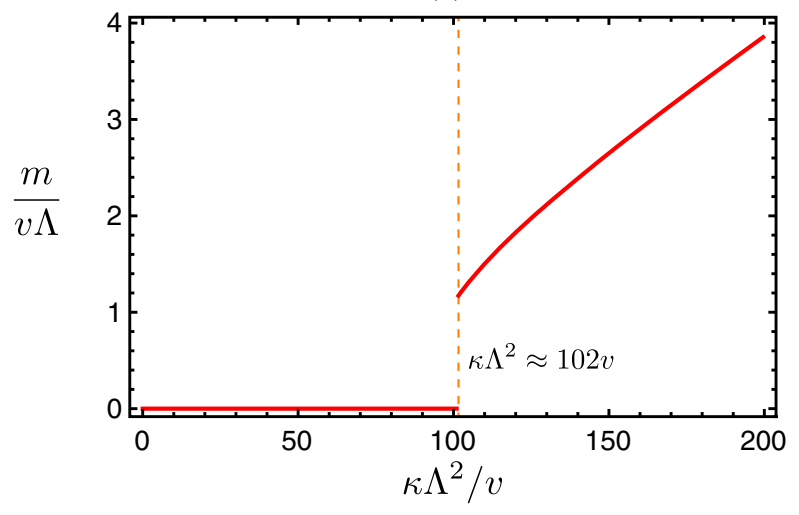

FIG. 13. (a) Variational energy difference $\Delta \mathcal{E}_{\text {trial }}$ [Eq. (A14)] versus mean-field mass $m$ for varying interaction strengths $\kappa$ in the continuum model given by Eqs. (16) and (17). For $\kappa \Lambda^{2} \gtrsim 102 v$, the variational energy is minimized with nonzero $\mathrm{m}$, signaling the spontaneous opening of a gap. (b) Optimal mass as a function of interaction strength. In the normalizations adopted above, $v$ is the velocity and $\Lambda$ is the cutoff.

$$
\mathcal{E}_{\text {trial }}=-\frac{m^{2}}{16 \pi v} I_{2}-\frac{\kappa m^{4}}{1024 \pi^{2} v^{4}}\left(I_{2}^{2}+I_{0} I_{2}\right) .
$$

We now minimize Eq. (A13) with respect to $m$. For $\kappa<0$, the minimization always yields $m=0$. With $\kappa>0$, however, a nontrivial solution does arise beyond a critical interaction strength. It is convenient to examine

$$
\Delta \mathcal{E}_{\text {trial }} \equiv \mathcal{E}_{\text {trial }}-\left.\mathcal{E}_{\text {trial }}\right|_{m=0}
$$

which quantifies the change in energy density due to a nonzero mass $m$. Figure 13 (a) plots $\Delta \mathcal{E}_{\text {trial }} /\left(v \Lambda^{2}\right)$ versus $m /(v \Lambda)$ for varying dimensionless interaction strengths $\kappa \Lambda^{2} / v$, while Fig. 13(b) displays the optimized mass as a function of $\kappa \Lambda^{2} / v$. A first-order jump in the optimal mass appears at a critical interaction strength

$$
\left(\kappa \Lambda^{2} / v\right)_{c} \approx 102
$$


The large numerical factor on the right-hand side naively suggests that spontaneous mass generation requires implausibly large interactions. We stress that such a conclusion is generally incorrect. In the next subsection, we see that lattice models with microscopic interaction strength $U_{\text {microscopic }}$ yield $\kappa=c U_{\text {microscopic }}$, where $c$ is a number in the range $10^{2}-10^{3}$. Thus, even modest microscopic interactions translate into "large" $\kappa$ values that can exceed the threshold in Eq. (A15), consistent with conclusions from more rigorous microscopic analyses reviewed in Secs. III A and III B.

\section{One-dimensional lattice model}

Next, we similarly study interacting Majorana fermions on an $N$-site chain with periodic boundary conditions governed by a Hamiltonian

$$
\begin{gathered}
H=H_{0}+\Delta H, \\
H_{0}=i t \sum_{a} \gamma_{a} \gamma_{a+1}, \\
\delta H=-g \sum_{a} \gamma_{a} \gamma_{a+m} \gamma_{a+n} \gamma_{a+p}
\end{gathered}
$$

with $t>0$ and $0<m<n<p$. We are specifically interested in quantifying the regime of interaction strength over which the chain spontaneously dimerizes. To this end, we employ a trial wave function given by the ground state of the free-fermion Hamiltonian

$$
H_{\mathrm{MF}}=i \sum_{a}\left[t+(-1)^{a} \bar{m}\right] \gamma_{a} \gamma_{a+1},
$$

where $\bar{m}$ is a variational parameter that, when nonzero, indicates spontaneous dimerization. Note that the translation $\gamma_{a} \rightarrow \gamma_{a+1}$ leaves $H$ invariant but flips the sign of $\bar{m}$ in $H_{\mathrm{MF}}$. The energies for configurations with $\bar{m}$ and $-\bar{m}$ thus necessarily match, so that we need consider only variational wave functions with $\bar{m} \geq 0$ in what follows.

The spectrum and eigenstates of Eq. (A19) can be readily constructed by going to momentum space. For our purposes here, it suffices to report correlation functions of Majorana bilinears $\left\langle\gamma_{a} \gamma_{b}\right\rangle$, from which all other correlations can be deduced using Wick's theorem. (All expectation values presented in this Appendix are taken with respect to the ground state of $H_{\mathrm{MF}}$.) In particular, we find

$$
\begin{aligned}
\left\langle\gamma_{a} \gamma_{b}\right\rangle= & \delta_{a b}+\left[(-1)^{a+b}-1\right] 4 i t f_{s, a-b} \\
& +\left[(-1)^{a}-(-1)^{b}\right] 4 i \bar{m} f_{c, a-b} .
\end{aligned}
$$

Above, we introduce functions

$$
f_{s, x}=\int_{-\pi / 2}^{\pi / 2} \frac{d k}{2 \pi} \frac{\sin k \sin (k x)}{E(k)},
$$

$$
f_{c, x}=\int_{-\pi / 2}^{\pi / 2} \frac{d k}{2 \pi} \frac{\cos k \cos (k x)}{E(k)},
$$

where $E(k)=\sqrt{(4 t \sin k)^{2}+(4 \bar{m} \cos k)^{2}}$ are the singleparticle excitation energies for $H_{\mathrm{MF}}$.

We can now straightforwardly obtain our trial energy density $\mathcal{E}_{\text {trial }}=\left\langle H_{0}+\delta H\right\rangle / N$. The hopping contribution is simply

$$
\frac{\left\langle H_{0}\right\rangle}{N}=-8 t^{2} f_{s, 1}
$$

The interaction term can be treated using the decomposition

$$
\begin{aligned}
\left\langle\gamma_{a} \gamma_{a+m} \gamma_{a+n} \gamma_{a+p}\right\rangle= & \left\langle\gamma_{a} \gamma_{a+m}\right\rangle\left\langle\gamma_{a+n} \gamma_{a+p}\right\rangle \\
& -\left\langle\gamma_{a} \gamma_{a+n}\right\rangle\left\langle\gamma_{a+m} \gamma_{a+p}\right\rangle \\
& +\left\langle\gamma_{a} \gamma_{a+p}\right\rangle\left\langle\gamma_{a+m} \gamma_{a+n}\right\rangle,
\end{aligned}
$$

yielding

$$
\frac{\langle\delta H\rangle}{N}=-16 g\left(F_{m n p}-F_{n m p}+F_{p m n}\right),
$$

where

$$
\begin{aligned}
F_{x y z}= & {\left[(-1)^{x}-1\right]\left\{\left[(-1)^{y+z}-1\right] t^{2} f_{s, x} f_{s, y-z}\right.} \\
& \left.+\left[(-1)^{y}-(-1)^{z}\right] \bar{m}^{2} f_{c, x} f_{c, y-z}\right\} .
\end{aligned}
$$

Our total trial energy density is then

$$
\mathcal{E}_{\text {trial }}=-8 t^{2} f_{s, 1}-16 g\left(F_{m n p}-F_{n m p}+F_{p m n}\right) .
$$

Below, it is also useful to consider the difference

$$
\Delta \mathcal{E}_{\text {trial }} \equiv \mathcal{E}_{\text {trial }}-\left.\mathcal{E}_{\text {trial }}\right|_{\bar{m}=0} .
$$

Let us specialize to the lattice model in Eq. (20) with $t^{\prime}=0$, for which $g=U$ and $m=1, n=3, p=4$. In this case, the trial energy density explicitly reads

$\mathcal{E}_{\text {trial }}=-8 t^{2} f_{s, 1}-64 U\left[t^{2}\left(f_{s, 3}^{2}-f_{s, 1}^{2}\right)+\bar{m}^{2}\left(f_{c, 1}^{2}-f_{c, 3}^{2}\right)\right]$.

At this point, it is instructive to relate Eq. (A29) to the energy density in Eq. (A13) for the continuum Hamiltonian. This exercise proceeds by (i) introducing a momentum cutoff in the $f_{s, x}$ and $f_{c, x}$ integrals, (ii) expanding the numerator in the integrands to order $k^{2}$, (iii) replacing $\sin k \rightarrow k$ and $\cos k \rightarrow 1$ in $E(k)$, and (iv) dropping a term proportional to $\bar{m}^{6} I_{2}^{2}$ that can arise only upon including higher-momentum terms in the continuum model. Some algebra yields the relations

$$
v=4 t, \quad m=8 \bar{m}, \quad \kappa=512 U .
$$


Note especially the large numerical prefactor in front of $U$ in connection with the discussion at the end of Appendix A 1.

We now minimize Eq. (A29) with respect to $\bar{m}$. Figure 14 (a) plots $\Delta \mathcal{E}_{\text {trial }} / t$ versus $\bar{m} / t$ for several $U / t$ values. The optimized $\bar{m}$ as a function of $U / t$ appears in Fig. 14(b). Our variational analysis predicts spontaneous dimerization-now via a continuous transition in contrast to the continuum model-for $U \gtrsim 0.29 t$. This prediction agrees reasonably well with DMRG, which yields spontaneous dimerization for $U \gtrsim 0.428 t$ [87]. Interestingly, for $U=t / 2$ our variational ansatz actually becomes exact since in this limit the interaction admits an exact selfconsistent mean-field decoupling; recall the discussion in Sec. III B. Figure 14(c) shows the optimized variational energy density together with the exact energy density extracted from Ref. [87], which indeed agree at $U=t / 2$.

Finally, suppose that we instead take $g=-U_{R}$ and $m=1, n=2, p=3$. This choice corresponds to a different interaction in which four adjacent Majorana fermions interact with strength $U_{R}$. We consider only $U_{R}>0$ in our variational analysis since negative $U_{R}$ values generate additional gapless modes beyond those in Eqs. (16) and (17) (see Appendix B for a synopsis); our trial wave functions are thus not expected to adequately capture the physics at $U_{R}<0$. The trial energy density for this model becomes

$$
\begin{aligned}
\mathcal{E}_{\text {trial }}= & -8 t^{2} f_{s, 1}-64 U_{R}\left[t^{2}\left(f_{s, 1}^{2}+f_{s, 1} f_{s, 3}\right)\right. \\
& \left.+\bar{m}^{2}\left(f_{c, 1}^{2}-f_{c, 1} f_{c, 3}\right)\right] .
\end{aligned}
$$

Upon connecting to the continuum energy density as we outline above, one finds the relations

$$
v=4 t, \quad m=8 \bar{m}, \quad \kappa=256 U_{R} .
$$

Once again, a large prefactor appears in the expression for $\kappa$ in agreement with the alternative derivation from Ref. [93]. Based on our continuum analysis, one might therefore expect that a spontaneous-dimerization transition sets in once $U_{R} / t>0$ becomes of order unity, as arises for the alternative interaction $U$. Curiously, however, minimizing the lattice energy density in Eq. (A31) yields an optimal $\bar{m}$ that vanishes for any $U_{R}>0$. Figure 15 illustrates this conclusion by plotting $\Delta \mathcal{E}_{\text {trial }} / t$ versus $\tan ^{-1}(\bar{m} / t)$ for varying $U_{R} / t$ values. More rigorous DMRG simulations do capture a dimerization transition but only at extremely large $U_{R} / t$. The following Appendix resolves the apparent discrepancy between our continuum and lattice analyses and explains the curious suppression of the instability for the $U_{R}$ interaction. (a)

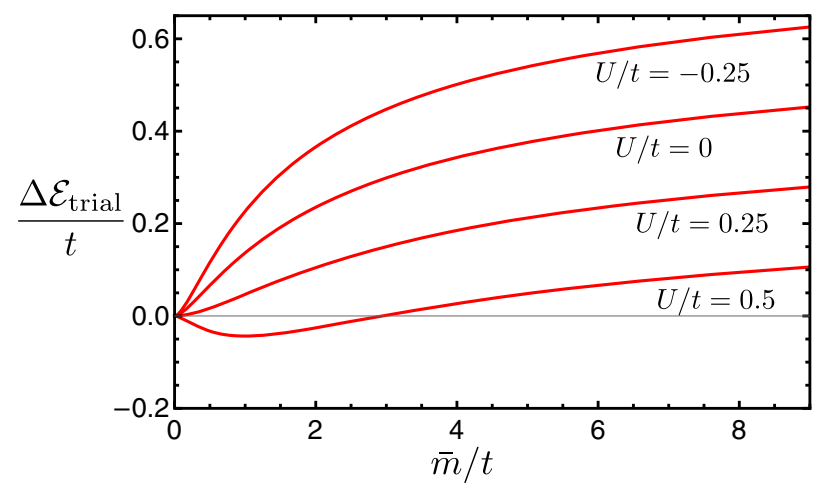

(b)

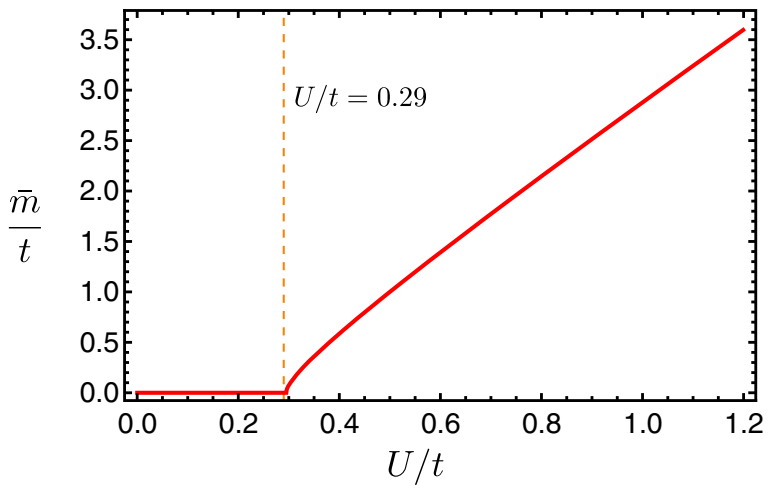

(c)

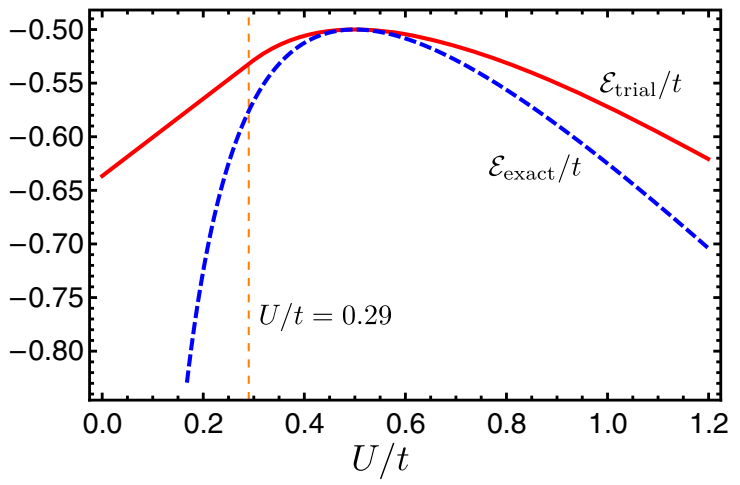

FIG. 14. Variational results for the lattice model given by Eqs. (A16) through (A18) with $m=1, n=3, p=4$, and $g=U$. (a) Variational energy difference $\Delta \mathcal{E}_{\text {trial }}$ [Eq. (A28)] versus mean-field dimerization order parameter $\bar{m}$ for varying interaction strengths $U$. At $U \gtrsim 0.29 t$, the variational energy is minimized by nonzero $\bar{m}$, indicating spontaneous dimerization (as captured by DMRG, but at slightly larger interaction strengths $U \gtrsim 0.428 t$ [87]). (b) Optimal variational $\bar{m}$ as a function of interaction strength. (c) Variational ground-state energy $\mathcal{E}_{\text {trial }}$ (solid red line) and exact ground-state energy $\mathcal{E}_{\text {exact }}$ (dashed blue line) [87] versus $U / t$. The two converge at $U=t / 2$, at which point the variational approach becomes exact. 


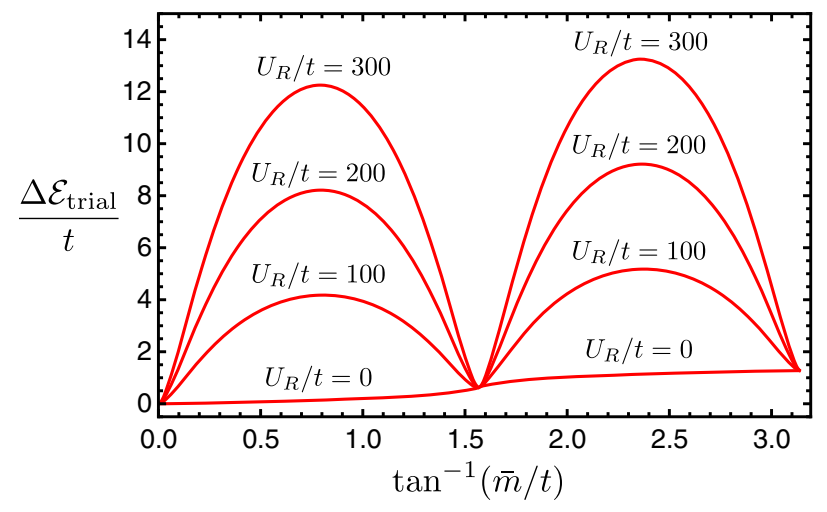

FIG. 15. Variational energetics for the lattice model given by Eqs. (A16) through (A18) with $m=1, n=2, p=3$, and $g=-U_{R}$. The plot shows the variational energy difference $\Delta \mathcal{E}_{\text {trial }}$ [Eq. (A28)] versus $\tan ^{-1}(\bar{m} / t)$, with $\bar{m}$ the mean-field dimerization order parameter, at various interaction strengths. For any $U_{R}$, the variational energy is minimized at $\bar{m}=0$ - precluding a transition at the mean-field level. DMRG calculations do capture a dimerization instability, but only at extremely strong interactions strengths $U_{R} / t \gtrsim 250[93,94]$. Appendix B explains the striking difference between the behavior of the two models examined here and in Fig. 14.

\section{APPENDIX B: INTERACTING MAJORANA FERMIONS IN AN ALTERNATIVE MICROSCOPIC MODEL}

Consider the microscopic model

$$
\begin{gathered}
H_{R}=H_{0}+\delta H, \\
H_{0}=i t \sum_{a} \gamma_{a} \gamma_{a+1}, \\
\delta H=U_{R} \sum_{a} \gamma_{a-1} \gamma_{a} \gamma_{a+1} \gamma_{a+2}
\end{gathered}
$$

defined with periodic boundary conditions. This model preserves both translation symmetry $T$ and chiral symmetry $\mathcal{C}$ defined in Sec. III B. References $[93,94,130]$ extensively studied the phase diagram; here we note the following features: (i) A single pair of gapless, counterpropagating Majorana fermions [Eq. (16)] captures the low-energy physics over the broad interval $-0.28 \lesssim U_{R} / t \lesssim 250$. Throughout, the central charge is $c=1 / 2$. (ii) The chain spontaneously dimerizes, thus gapping the Majorana fermions, for $U_{R} / t \gtrsim 250$ - which again reflects a vastly stronger interaction strength compared to that required for dimerization in Eq. (20). (iii) For $-2.86 \lesssim U_{R} / t \lesssim-0.285$, the chain remains gapless, but the low-energy physics is described by three pairs of counterpropagating Majorana fermions. The central charge accordingly becomes $c=3 / 2$. Below, we explain, within a unified framework, both the extreme robustness of the $c=1 / 2$ phase to interactions as well as the onset of the $c=3 / 2$ phase.
Let us start from the noninteracting limit, $U_{R}=0$. Here, the chain is diagonalized by passing to momentum space via

$$
\gamma_{a}=\frac{\sqrt{2}}{\sqrt{N}} \sum_{k} e^{i k a} \gamma_{k}
$$

with $N$ the number of Majorana sites in the chain. In our conventions, $\left\{\gamma_{k}, \gamma_{k^{\prime}}\right\}=\delta_{k,-k^{\prime}}$. Note also that selfHermiticity of $\gamma_{a}$ implies that $\gamma_{k}=\gamma_{-k}^{\dagger}$; this relation allows a complete description of the chain using operators acting in half of the Brillouin zone. In particular, one finds

$$
H_{0}=\sum_{-\pi<k<0} \epsilon_{0}(k) \gamma_{k}^{\dagger} \gamma_{k},
$$

where

$$
\epsilon_{0}(k)=4 t|\sin k|
$$

is the Majorana-fermion kinetic energy. The ground state follows by taking $\gamma_{k}^{\dagger} \gamma_{k}=0$ for all momenta in the left half of the Brillouin zone. Single-particle excitations are obtained by taking $\gamma_{p}^{\dagger} \gamma_{p}=1$ for some momentum $p$, incurring an energy cost of $\epsilon_{0}(p)$. In the low-energy theory, left- and right-moving Majorana fermions correspond to excitations near momentum 0 and $-\pi$, respectively.

Interactions can generate additional symmetry-allowed hopping processes, as already observed in Ref. [94], which in turn, renormalize the kinetic energy. Reference [94] explored these effects within self-consistent mean-field theory. We instead capture kinetic energy renormalization via an exact rewriting of interactions analogous to normal ordering. Specifically, we organize four-fermion terms so that all matrix elements vanish identically in the subspace consisting of states with either zero or one single-particle excitation. To obtain this form, one can first express $\delta H$ in terms of Majorana operators with momenta in the left Brillouin zone half, and then use anticommutation relations to move all $\gamma_{k}^{\dagger}$ operators to the left of $\gamma_{k}$. The final expression takes the form

$$
\delta H=\delta H_{\mathrm{int}}+\delta H_{\mathrm{KE}},
$$

where $\delta H_{\text {int }}$ vanishes within the zero- and one-excitation subspace as desired and $\delta H_{\mathrm{KE}}$ contains the kinetic energy renormalization. After some algebra, we explicitly find

$$
\begin{gathered}
\delta H_{\mathrm{KE}}=\sum_{-\pi<k<0} \delta \epsilon(k) \gamma_{k}^{\dagger} \gamma_{k}, \\
\delta \epsilon(k)=U_{R}\left[\frac{56}{3 \pi}|\sin k|-\frac{8}{\pi} \sin (3 k)\right] .
\end{gathered}
$$


The first term in Eq. (B9) reflects renormalization of the nearest-neighbor hopping amplitude $t$, while the second represents an interaction-induced third-neighbor hopping.

The decomposition of interactions employed above is very useful, at least for sufficiently small $\left|U_{R}\right| / t$, since the expectation value of the energy for single-excitation states can be read off immediately. Specifically, upon including the bare component, the total kinetic energy for an excitation with momentum $k$ becomes $\epsilon(k) \equiv \epsilon_{0}(k)+\delta \epsilon(k)$. The dispersion for right- and left-moving Majorana fermions in the continuum limit follows from expanding $\epsilon(k)$ near $k=-\pi$ and 0 ; one finds $\epsilon \sim \pm v k$ with

$$
v=4 t+\frac{128}{3 \pi} U_{R}
$$

the renormalized velocity.

For $U_{R}<0$, interactions reduce $v$, and at a critical value $U_{R}^{*}=-(3 \pi / 32) t \approx-0.295 t$ the velocity vanishes. Below this value, the renormalized kinetic energy $\epsilon(k)$ supports additional pairs of gapless Majorana fermions-changing the central charge from $c=1 / 2$ to $3 / 2$. Remarkably, the critical interaction strength $U_{R}^{*}$ extracted from our treatment agrees quantitatively with DMRG predictions. We note that the mean-field treatment performed in Ref. [94] recovers similar quantitative agreement.

For $U_{R}>0$, interactions instead enhance $v$. Recall from Sec. III A that in the low-energy description, the critical phase with central charge $c=1 / 2$ becomes unstable to spontaneous mass generation when $\kappa \Lambda^{2} / v$ becomes of order unity, where $\Lambda$ is a momentum cutoff and $\kappa$ is the coupling from Eq. (17). In the present context, we have $\kappa \propto U_{R}$. Upward renormalization of $v$ clearly boosts the robustness of the $c=1 / 2$ phase against interactions, though we are unable to quantitatively obtain the critical value of $U_{R} \sim 250$ at which DMRG finds an instability.

We can, nevertheless, stringently test the scenario above. If kinetic energy renormalization indeed pushes the spontaneous-dimerization transition to extremely large $U_{R}$ values, then removing this renormalization should reduce the critical $U_{R}$ by 3 orders of magnitude. Remarkably, we indeed find such a dramatic reduction. Consider the modified Hamiltonian

$$
H_{R}^{\prime}=H_{R}-\delta H_{\mathrm{KE}},
$$

which is identical to Eq. (B1) except that the kinetic energy renormalization is subtracted off, thus yielding a $U_{R^{-}}$ independent velocity. DMRG simulations find a transition to a gapped phase in this model at $U_{R} / t \approx 0.48098(1)$, comparable to the critical interaction strength obtained from the alternative model in Eq. (20) at $t^{\prime}=0$. See Fig. 16. Furthermore, at least over the range of $U_{R}$ shown in the figure, the transition to a $c=3 / 2$ phase at $U_{R}<0$ has also been removed (as expected upon removal of the kinetic energy renormalization).

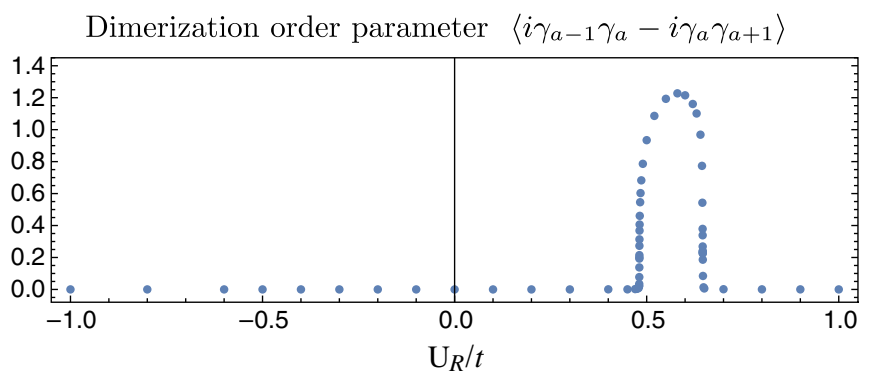

FIG. 16. Dimerization order parameter $\left\langle i \gamma_{a-1} \gamma_{a}-i \gamma_{a} \gamma_{a+1}\right\rangle$ versus $U_{R} / t$ obtained from DMRG simulations of the Hamiltonian in Eq. (B11). This Hamiltonian is the same as Eq. (B1) but with the interaction-induced kinetic energy renormalization subtracted off. The subtraction reduces the critical interaction strength required for spontaneous dimerization by 3 orders of magnitude-from $U_{R} / t \approx 250$ down to $U_{R} / t \approx 0.48$. Outside of the dome-shaped dimerized region, the system realizes a gapless state with central charge $c=1 / 2$ (at least over the $U_{R} / t$ window shown). Reentrance of the $c=1 / 2$ critical state at $U_{R} / t \gtrsim 0.65$ can also be understood from the subtraction, as we explain in Appendix B.

Conspicuously, Fig. 16 also reveals a reentrant $c=1 / 2$ critical phase for $U_{R} / t=0.64585(3)$. We can explain this feature as well by examining Eq. (B11), which can equivalently be written as

$$
\begin{aligned}
H_{R}^{\prime}= & i t \sum_{a} \gamma_{a} \gamma_{a+1}+U_{R} \sum_{a}\left[\gamma_{a-1} \gamma_{a} \gamma_{a+1} \gamma_{a+2}\right. \\
& \left.-\frac{2 i}{\pi}\left(\frac{7}{3} \gamma_{a} \gamma_{a+1}+\gamma_{a} \gamma_{a+3}\right)\right] .
\end{aligned}
$$

Up to moderate values of $U_{R} / t$, it is natural to interpret the second line-which is just $\delta H_{\mathrm{KE}}$ expressed in real spaceas a correction to the bare kinetic energy on the first line. However, at sufficiently large $U_{R} / t$ this "correction" overwhelms the bare piece, suggesting the following alternative viewpoint. Let us trivially rewrite $H_{R}^{\prime}$ as

$$
\begin{aligned}
H_{R}^{\prime}= & \sum_{a}\left[i t \gamma_{a} \gamma_{a+1}-\frac{4 i}{\pi} U_{R}\left(\frac{7}{3} \gamma_{a} \gamma_{a+1}+\gamma_{a} \gamma_{a+3}\right)\right] \\
& +U_{R} \sum_{a}\left[\gamma_{a-1} \gamma_{a} \gamma_{a+1} \gamma_{a+2}\right. \\
& \left.+\frac{2 i}{\pi}\left(\frac{7}{3} \gamma_{a} \gamma_{a+1}+\gamma_{a} \gamma_{a+3}\right)\right],
\end{aligned}
$$

and view the top line as our new "bare" kinetic term. For $U_{R} / t>3 \pi / 16 \approx 0.59$, all of the corresponding kinetic energy eigenvalues flip sign compared to the kinetic energy from the $t$ term alone-thus completely changing the character of the associated noninteracting ground state. The second and third lines represent an interaction that has no nontrivial matrix elements in the subspace with zero or 
one single-particle excitations about that modified ground state. [Note the relative sign between the final terms in Eqs. (B12) and (B13).] In this $U_{R} / t$ regime, the top line yields a velocity for right and left movers of $v=(256 / 3 \pi) U_{R}-4 t$. Once again, we end up with a kinetic energy scale that grows with $U_{R}$, so that the dimerization instability is naturally suppressed beyond a critical value of $U_{R} / t$ as observed in DMRG.

As an additional sanity check, one can extract kinetic energy renormalization arising from the four-fermion interaction in Eq. (20) via exactly the same procedure leading to Eq. (B7) above. The result takes the form in Eq. (B8) where now

$$
\delta \epsilon(k)=-\frac{64}{3 \pi} U\left|\sin ^{3} k\right| .
$$

Near $k=0$ and $-\pi, \delta \epsilon(k) \propto k^{3}$, indicating that velocity renormalization vanishes in this model. Thus, no such suppression of the dimerization instability is expected in our scenario [131], and indeed, a transition occurs at the modest value $U \approx 0.428 t$ [87]. Reference [87] further studied Eq. (B1) with $\delta H$ replaced by yet another interaction,

$\delta H^{\prime}=U_{y} \sum_{a}\left(\gamma_{a-2} \gamma_{a} \gamma_{a+1} \gamma_{a+2}-\gamma_{a-2} \gamma_{a-1} \gamma_{a} \gamma_{a+2}\right)$.

In this case, we find that $\delta \epsilon(k)=0$-i.e., $U_{y}$ produces no kinetic energy renormalization at all. DMRG simulations find a transition at a similarly modest value $U_{y} \approx 0.45 t$ [87]. Together, the results above strongly support our explanation for the anomalously strong interaction strength required for dimerization in Eq. (B1), which has heretofore remained enigmatic.

Zooming out, we see from this discussion that microscopic details matter when dealing with instabilities arising from "strong" interactions that are irrelevant at weak coupling. The insights we obtain here can potentially be exploited to concoct new models that, depending on the desired outcome, either enhance or suppress the effects of such strong irrelevant interactions.

\section{APPENDIX C: ANALYSIS OF FERMION TUNNELING ACROSS A CONSTRICTION}

Here we study the interferometer in Fig. 10 in the limit where only fermions are allowed to tunnel across the constriction. That is, we take the fermion-tunneling amplitude $t_{\psi} \neq 0$ but set $t_{\sigma}=0$ in Eq. (34)—in which case, we arrive at a free-fermion scattering problem that admits an exact solution. Our goal is to deduce the phase $e^{i \phi_{\text {emergent }}}$ acquired by an emergent fermion that travels from position $x_{0}$ before the constriction to position $x_{3}$ after the constriction. Figure 17(a) illustrates the interferometer geometry, while Fig. 17(b) shows an "unfolded" version. For (a)

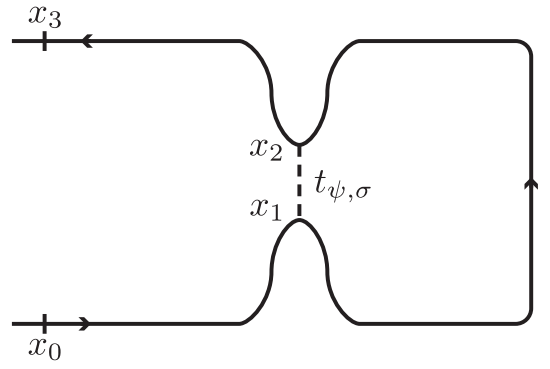

(b)

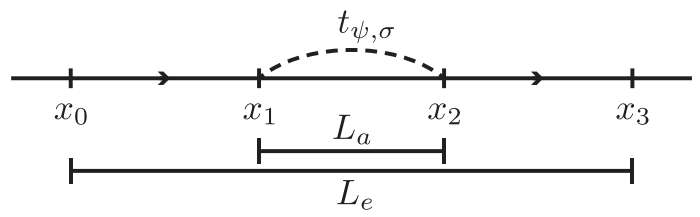

FIG. 17. (a) Geometry and coordinates used to explicitly analyze the emergent-fermion transmission amplitude in the interferometer from Fig. 10. Appendixes C and D, respectively, treat the cases where only fermions tunnel (with coupling $t_{\psi}$ ) and only Ising anyons tunnel (with coupling $t_{\sigma}$ ) across the constriction. (b) Unfolded version of (a), not to scale. In terms of lengths shown in Fig. 10(a), we have $x_{2}-x_{1}=L_{a}$ and $x_{3}-x_{0}=L_{e}$.

simplicity, we consider the case where no nontrivial quasiparticles reside in the bulk of the interferometer; i.e., we assume $a=I$ in Fig. 10.

Evaluating the Heisenberg equation of motion $\partial_{t} \gamma=$ $i\left[\mathcal{H}_{0}+\mathcal{H}_{\text {tun }}, \gamma\right]$, with $\mathcal{H}_{0}$ the chiral Majorana kinetic energy, one finds

$$
\begin{aligned}
\partial_{t} \gamma(x, t)= & -v_{e} \partial_{x} \gamma(x, t)+\frac{i}{2} t_{\psi} e^{-i \pi h_{\psi}}\left[\delta\left(x-x_{1}\right) \gamma\left(x_{2}, t\right)\right. \\
& \left.-\delta\left(x-x_{2}\right) \gamma\left(x_{1}, t\right)\right] .
\end{aligned}
$$

As before, $v_{e}$ is the emergent-fermion edge velocity, while $x_{1}$ and $x_{2}$ respectively denote positions on the lower and upper sides of the constriction (see again Fig. 17). By solving the equation of motion, one can relate $\gamma\left(x_{3}, t\right)$ to $\gamma\left(x_{0}, 0\right)$. The phase of interest follows from the equal-time relation $\gamma\left(x_{3}, 0\right)=e^{i \phi_{\text {emergent }}} \gamma\left(x_{0}, 0\right)$, so hereafter we focus on the solution at $t=0$.

Away from $x=x_{1,2}$, Eq. (C1) reduces to a standard chiral wave equation. Suppose that $x_{j}^{+}$denotes a coordinate slightly larger than $x_{j}$, while $x_{j}^{-}$denotes a coordinate slightly smaller than $x_{j}$, and let $k_{e}$ be the incident emergent-fermion momentum. One immediately finds

$$
\begin{aligned}
& \gamma\left(x_{1}^{-}, 0\right)=e^{i k_{e}\left(x_{1}-x_{0}\right)} \gamma\left(x_{0}, 0\right), \\
& \gamma\left(x_{2}^{-}, 0\right)=e^{i k_{e}\left(x_{2}-x_{1}\right)} \gamma\left(x_{1}^{+}, 0\right),
\end{aligned}
$$




$$
\gamma\left(x_{3}, 0\right)=e^{i k_{e}\left(x_{3}-x_{2}\right)} \gamma\left(x_{2}^{+}, 0\right) .
$$

(In the exponentials above, we replace $x_{j}^{ \pm} \rightarrow x_{j}$ since the difference is inconsequential.) Next, integrating Eq. (C1) over a small region enclosing $x_{1}$ and similarly for $x_{2}$ yields the linear relations

$0=\gamma\left(x_{1}^{+}, 0\right)-\gamma\left(x_{1}^{-}, 0\right)+\tilde{t}_{\psi}\left[\gamma\left(x_{2}^{+}, 0\right)+\gamma\left(x_{2}^{-}, 0\right)\right]$,

$0=\gamma\left(x_{2}^{+}, 0\right)-\gamma\left(x_{2}^{-}, 0\right)-\tilde{t}_{\psi}\left[\gamma\left(x_{1}^{+}, 0\right)+\gamma\left(x_{1}^{-}, 0\right)\right]$,

where $\tilde{t}_{\psi}=-i\left(t_{\psi} / 4 v_{e}\right) e^{-i \pi h_{\psi}}$. Combining with Eq. (C3) and defining $e^{i \chi}=e^{i k_{e}\left(x_{2}-x_{1}\right)}$, one obtains

$$
\gamma\left(x_{2}^{+}, 0\right)=e^{i \chi} \frac{1+2 e^{-i \chi} \tilde{t}_{\psi}+\tilde{t}_{\psi}^{2}}{1+2 e^{i \chi} \tilde{t}_{\psi}+\tilde{t}_{\psi}^{2}} \gamma\left(x_{1}^{-}, 0\right)
$$

Finally, Eqs. (C2), (C4), and (C7) together imply that $\gamma\left(x_{3}, 0\right)=e^{i \phi_{\text {emergent }}} \gamma\left(x_{0}, 0\right)$ with

$$
e^{i \phi_{\text {emergent }}}=e^{i k_{e}\left(x_{3}-x_{0}\right)} \frac{1+2 e^{-i \chi} \tilde{t}_{\psi}+\tilde{t}_{\psi}^{2}}{1+2 e^{i \chi} \tilde{t}_{\psi}+\tilde{t}_{\psi}^{2}} .
$$

To make contact with Sec. VII A from the main text, we now set $x_{3}-x_{0}=L_{e}$ and $x_{2}-x_{1}=L_{a}$. Additionally, we expand Eq. (C8) to first order in $\tilde{t}_{\psi}$ and use $i e^{-i \pi h_{\psi}}=1$, leading to

$$
e^{i \phi_{\text {emergent }}} \approx e^{i k_{e} L_{e}}\left[1+\frac{t_{\psi}}{2 v_{e}}\left(-e^{-i k_{e} L_{a}}+e^{i k_{e} L_{a}}\right)\right] .
$$

The three terms in brackets respectively correspond to paths (i), (ii), and (iii) discussed in Sec. VII A. From this explicit calculation, we can trace the relative minus sign between the terms for paths (ii) and (iii) to the anticommutation relations obeyed by the Majorana fermions. Comparing to Eqs. (42) and (53), the weights $w_{i i}$ and $w_{i i i}$ are given by Eq. (43) with $\alpha_{i i}=-\alpha_{i i i}=-1 / 2$, as quoted in Eq. (45).

\section{APPENDIX D: ANALYSIS OF ISING-ANYON TUNNELING ACROSS A CONSTRICTION}

In this Appendix, we continue to study the interferometer in Fig. 10, but now allowing only Ising anyons to tunnel across the constriction. The geometry and coordinates used are again given in Fig. 17. We evaluate the transmission amplitude describing propagation of an emergent fermion from position to $x_{0}$ to $x_{3}$ perturbatively in Ising-anyon tunneling, assuming that the interferometer does not contain any nontrivial bulk quasiparticles as in Appendix C.

\section{Hamiltonian and conventions}

We set $t_{\psi}=0$ but take $t_{\sigma} \neq 0$ in Eq. (34), so that the full Hamiltonian becomes

$$
\begin{aligned}
\mathcal{H} & =\mathcal{H}_{0}+\mathcal{H}_{\text {tun }}, \\
\mathcal{H}_{\text {tun }} & =e^{-i \pi h_{\sigma}} t_{\sigma} \sigma\left(x_{2}\right) \sigma\left(x_{1}\right) .
\end{aligned}
$$

Precisely as in Appendix $\mathrm{C}, \mathcal{H}_{0}$ describes a chiral rightmoving free Majorana fermion. In the tunneling term, $h_{\sigma}=1 / 16$ is the conformal weight (spin) of the $\sigma$ field and $t_{\sigma} \in \mathbb{R}$ is the coupling coefficient. For the remainder of this Appendix we set the velocity $v_{e}=1$.

We choose $\sigma$ to be Hermitian and normalized such that

$$
\left\langle\sigma\left(x^{\prime}, t^{\prime}\right) \sigma(x, t)\right\rangle_{0}=\frac{1}{\left(i t^{\prime}-i x^{\prime}-i t+i x\right)^{1 / 8}} .
$$

The subscript of the correlation function $\langle\cdots\rangle_{0}$ indicates that the correlator is computed with respect to the free CFT Hamiltonian $\mathcal{H}_{0}$. [The choice of phase on the right side of Eq. (D2) guarantees that the correlator $\langle\sigma(0,-i \beta) \sigma(0)\rangle=$ $\operatorname{Tr}\left[\sigma(0) e^{-\beta H} \sigma(0)\right] / \operatorname{Tr} e^{-\beta H}$ is positive-a necessary condition for $\sigma=\sigma^{\dagger}$.] It is straightforward to check that $\mathcal{H}_{\text {tun }}$ is indeed Hermitian. Likewise, we define the normalized Majorana field

$$
\psi=\sqrt{4 \pi} \gamma,
$$

such that

$$
\left\langle\psi\left(x^{\prime}, t^{\prime}\right) \psi(x, t)\right\rangle_{0}=\frac{1}{\left(i t^{\prime}-i x^{\prime}-i t+i x\right)} .
$$

\section{Scattering states}

Next, we specify the formalism used to compute the transmission amplitude. We quantize the CFT along slices at fixed positions $x$; wave functions are written on "position slices" that live for all of time. (One should contrast to the usual framework wherein states live on fixed time slices.) Such a reformulation is, in principle, applicable to all field theories, but is particularly convenient for CFTs due to the symmetry between space and time coordinates [132]. An interaction term may manifest itself in different ways within this rotated frame. For example, a point defect localized in space become a global (instantaneous) quantum quench.

In the same spirit as Eq. (D1), we decompose the action into its free part and an interaction part:

$$
S=\int P_{0} d x+S_{\text {tun }} .
$$


The operator $P_{0}$ generates spatial translations of the free CFT, while $S_{\text {tun }}$ consists of a spatially nonlocal global term (spanning all of time),

$$
\begin{aligned}
S_{\text {tun }} & =-\int_{-\infty}^{\infty} d t \mathcal{H}_{\mathrm{tun}}(t) \\
& =-e^{-i \pi h_{\sigma}} t_{\sigma} \int_{-\infty}^{\infty} d t \sigma\left(x_{2}, t\right) \sigma\left(x_{1}, t\right) .
\end{aligned}
$$

For the problem at hand, the tunnel junction "teleports" particles between positions $x_{1}$ and $x_{2}$ and can be interpreted as a wormhole allowing "time travel" between the two positions. (This nonlocality makes it difficult to write the corresponding momentum operator for the tunneling term.) We choose to work in the interaction picture, where operators are related to those in the Schrödinger picture via

$$
O(x, t)=e^{-i P_{0} x} O(t) e^{i P_{0} x} .
$$

The state describing an incoming emergent fermion with positive frequency $\omega$ is written as

$$
\left|\psi_{\omega}\right\rangle=\frac{1}{2 \pi} \int_{-\infty}^{\infty} d t e^{-i \omega t} \psi(t)|0\rangle,
$$

where $|0\rangle$ is the ground state of $P_{0}$. This state exhibits the normalization

$$
\left\langle\psi_{\omega^{\prime}} \mid \psi_{\omega}\right\rangle=\delta\left(\omega-\omega^{\prime}\right)
$$

and carries momentum

$$
P_{0}\left|\psi_{\omega}\right\rangle=\omega\left|\psi_{\omega}\right\rangle .
$$

Recall that we set the pesky velocity to unity; hence, here and below, $\omega$ corresponds to $k_{e}$ from Sec. VII A.

Let $A\left(\omega ; x_{0}, x_{3}\right)$ denote the amplitude for transmission of the Majorana fermion from position $x_{0}$ to $x_{3}$ at frequency $\omega$. In terms of the field theory, $A$ is the quantum amplitude associated with the spatial strip $\left[x_{0}, x_{3}\right]$ with boundary conditions set by the incoming/outgoing states. Formally, the amplitude is defined via

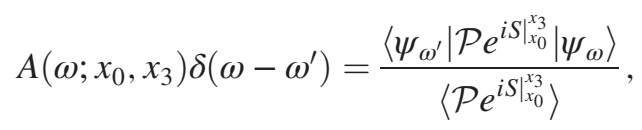

where $\left.S\right|_{x} ^{y}$ is the action [Eq. (D5)] restricted to the spatial interval $[x, y]$, and $\mathcal{P}$ denotes path ordering (of the position coordinates). We expand $A$ in powers of $t_{\sigma}$,

$$
A=A^{(0)}+t_{\sigma} A^{(1)}+t_{\sigma}^{2} A^{(2)}+\ldots,
$$

so that $A^{(n)}$ captures the $n$ th-order correction in the perturbative series.

The zeroth-order piece follows from free propagation of the scattering states, i.e., evolving $\psi(x, t)$ with the $t_{\sigma}=0$ Hamiltonian:

$$
\begin{aligned}
A^{(0)}\left(\omega ; x_{3}, x_{0}\right) \delta\left(\omega-\omega^{\prime}\right) & =\frac{\left\langle\psi_{\omega^{\prime}}\left|e^{i P_{0}\left(x_{3}-x_{0}\right)}\right| \psi_{\omega}\right\rangle_{0}}{\left\langle e^{i P_{0}\left(x_{3}-x_{0}\right)}\right\rangle_{0}} \\
& =e^{i \omega\left(x_{3}-x_{0}\right)}\left\langle\psi_{\omega^{\prime}} \mid \psi_{\omega}\right\rangle_{0} .
\end{aligned}
$$

We thus obtain the expected result $A^{(0)}\left(\omega ; x_{3}, x_{0}\right)=$ $e^{i \omega\left(x_{3}-x_{0}\right)}$.

The first-order correction to the amplitude is

$$
\begin{aligned}
& t_{\sigma} A^{(1)}\left(\omega ; x_{3}, x_{0}\right) \delta\left(\omega-\omega^{\prime}\right)=-i\left\langle\psi_{\omega^{\prime}}\left(x_{3}\right)\left|\int_{-\infty}^{\infty} d t^{\prime}\left[\mathcal{H}_{\text {tun }}\left(t^{\prime}\right)-\left\langle\mathcal{H}_{\text {tun }}\right\rangle_{0}\right]\right| \psi_{\omega}\left(x_{0}\right)\right\rangle_{0} \\
& =-i t_{\sigma} e^{-i \pi h_{\sigma}}\left\langle\psi_{\omega^{\prime}}\left(x_{3}\right)\left|\int_{-\infty}^{\infty} d t^{\prime}\left[\sigma\left(x_{2}, t^{\prime}\right) \sigma\left(x_{1}, t^{\prime}\right)-\Delta\right]\right| \psi_{\omega}\left(x_{0}\right)\right\rangle_{0} .
\end{aligned}
$$

Here, $\Delta$ is a constant, defined through $t_{\sigma} e^{-i \pi h_{\sigma}} \Delta=\left\langle\mathcal{H}_{\text {tun }}\right\rangle_{0}$, chosen to cancel off the phase correction to the vacuum. We also let $\left|\psi_{\omega}(x)\right\rangle=\int(d t / 2 \pi) e^{-i \omega t} \psi(x, t)|0\rangle$ denote a (temporal) plane wave at position $x$. Expanding the scattering states in terms of their integral definitions and the operators in the Schrödinger picture yields

$$
A^{(1)}\left(\omega ; x_{3}, x_{0}\right) \delta\left(\omega-\omega^{\prime}\right)=\frac{-i e^{-i \pi h_{\sigma}}}{(2 \pi)^{2}} \int_{t_{2}, t_{1}, t^{\prime}} e^{i\left(\omega^{\prime} t_{2}-\omega t_{1}\right)}\left\langle\psi\left(t_{2}\right) e^{i P\left(x_{3}-x_{2}\right)}\left[\sigma\left(t^{\prime}\right) e^{i P L_{a}} \sigma\left(t^{\prime}\right)-\Delta e^{i P L_{a}}\right] e^{i P\left(x_{1}-x_{0}\right)} \psi\left(t_{1}\right)\right\rangle_{0} .
$$

Above, we use $x_{2}-x_{1}=L_{a}$. As the scattering states are eigenstates of the momentum operator, we can replace $e^{i P\left(x_{3}-x_{2}\right)}$ and $e^{i P\left(x_{1}-x_{0}\right)}$ with their respective eigenvalues $e^{i \omega^{\prime}\left(x_{3}-x_{2}\right)}$ and $e^{i \omega\left(x_{1}-x_{0}\right)}$. In addition, we can eliminate the factor $\delta\left(\omega-\omega^{\prime}\right)$ on 
the left-hand side by integrating over $\omega$ on both sides; doing so fixes $t_{1}=0$ and eliminates one of the integrals on the righthand side. With some simple substitution of variables, the first-order amplitude correction can now be written as

$$
A^{(1)}\left(\omega ; x_{3}, x_{0}\right)=-\frac{i e^{-i \pi h_{\sigma}} e^{i \omega\left(x_{3}-x_{0}-L_{a}\right)}}{2 \pi} \int_{t_{2}, t^{\prime}} e^{i \omega t_{2}}\left\langle\psi\left(t_{2}\right)\left[\sigma\left(t^{\prime}\right) e^{i P L_{a}} \sigma\left(t^{\prime}\right)-\Delta e^{i P L_{a}}\right] \psi(0)\right\rangle_{0} .
$$

From Eq. (D2), one finds $\Delta=\left\langle\sigma\left(t^{\prime}\right) e^{i P L_{a}} \sigma\left(t^{\prime}\right)\right\rangle_{0}=\left(-i L_{a}\right)^{-1 / 8}$.

\section{Evaluation of the first-order correction}

We now evaluate the first-order contribution $A^{(1)}(\omega)$ by first integrating over $t^{\prime}$ and then integrating over $t_{2}$. Let

$$
\begin{aligned}
\mathcal{I}\left(t^{\prime}\right) & =\left\langle\psi\left(t_{2}-i \epsilon\right)\left[\sigma\left(t^{\prime}\right) e^{i P L_{a}} \sigma\left(t^{\prime}\right)-\Delta e^{i P L_{a}}\right] \psi(i \epsilon)\right\rangle_{0} \\
& =\left\langle\psi\left(t_{2}-L_{a}-i \epsilon\right) \sigma\left(t^{\prime}-L_{a}\right) \sigma\left(t^{\prime}\right) \psi(i \epsilon)\right\rangle_{0}-\left\langle\sigma\left(t^{\prime}-L_{a}\right) \sigma\left(t^{\prime}\right)\right\rangle_{0}\left\langle\psi\left(t_{2}-L_{a}-i \epsilon\right) \psi(i \epsilon)\right\rangle_{0}
\end{aligned}
$$

be the regulated form the integrand in Eq. (D16) (without the $e^{i \omega t_{2}}$ factor). We take $\epsilon \rightarrow 0^{+}$at the end of the calculation. The correlation function $\mathcal{I}$ can be computed via standard CFT techniques [4,133]. For instance, one can evaluate $\left\langle\psi\left(z_{1}\right) \sigma\left(z_{2}\right) \sigma\left(z_{3}\right) \psi\left(z_{4}\right)\right\rangle$ using a conformal transformation that maps the plane to a cylinder, placing the $\sigma$ fields at $t= \pm \infty$. The correlator then reduces to computing the two-point correlation function $\left\langle\psi(x, t) \psi\left(x^{\prime}, t^{\prime}\right)\right\rangle_{\text {cyl }}$ with the fermion field $\psi$ having periodic boundary conditions. Undoing the conformal transformation yields the desired result:

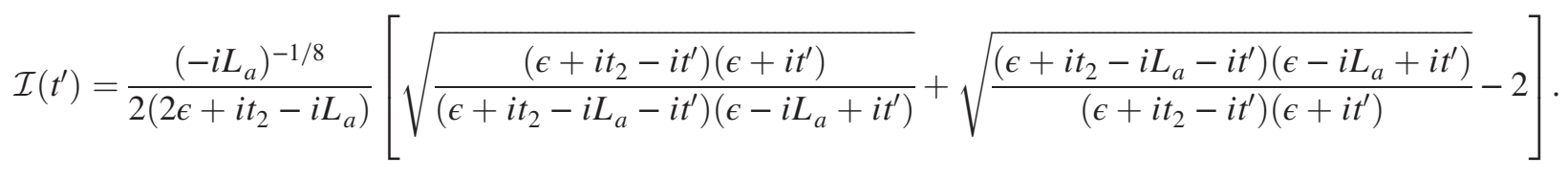

Notice that $\int_{t^{\prime}} \mathcal{I}$ is absolutely convergent since $\mathcal{I}\left(t^{\prime}\right)$ decays as $\mathcal{O}\left(\left(t^{\prime}\right)^{-4}\right)$ for large $t^{\prime}$. This convergence results from the $\Delta$ subtraction (corresponding to the -2 term in brackets), which eliminates the leading contribution in $\mathcal{I}\left(t^{\prime}\right)$.

The function $\mathcal{I}\left(t^{\prime}\right)$ has four branch points at $t_{2}-L_{a}-i \epsilon, t_{2}-i \epsilon, i \epsilon$, and $L_{a}+i \epsilon$. Observe that the first two sit below the real axis, while the latter two sit above the real axis. The $\int d t^{\prime}$ integral is to be evaluated with a branch cut connecting the two upper branch points, and a branch cut connecting the two lower points, such that $\mathcal{I}$ is analytic along the real line. To simplify the terms in brackets, we introduce a shift of variables $t^{\prime} \mapsto t^{\prime}+\left(t_{2} / 2\right)$ :

$$
\mathcal{I}\left(t^{\prime}+\frac{t_{2}}{2}\right)=\frac{1}{2\left(-i L_{a}\right)^{1 / 8}\left(2 \epsilon+i t_{2}-i L_{a}\right)}\left[\sqrt{\frac{\left(t^{\prime}\right)^{2}+a^{2}}{\left(t^{\prime}\right)^{2}+b^{2}}}+\sqrt{\frac{\left(t^{\prime}\right)^{2}+b^{2}}{\left(t^{\prime}\right)^{2}+a^{2}}}-2\right]
$$

with $a=\epsilon+i t_{2} / 2, b=\epsilon+i t_{2} / 2-i L_{a}$. At this point, we can utilize the integral identity in Eq. (E5) from Appendix E to write

$$
\begin{aligned}
\int_{-\infty}^{\infty} d t^{\prime} \mathcal{I}\left(t^{\prime}+\frac{t_{2}}{2}\right) & =\frac{1}{2\left(-i L_{a}\right)^{1 / 8}\left(2 \epsilon+i t_{2}-i L_{a}\right)} \times 4(a+b)\left[K\left(\frac{a-b}{a+b}\right)-E\left(\frac{a-b}{a+b}\right)\right] . \\
& =\frac{2 i^{1 / 8}}{L_{a}^{1 / 8}}\left[K\left(\frac{L_{a}}{t_{2}-L_{a}-2 i \epsilon}\right)-E\left(\frac{L_{a}}{t_{2}-L_{a}-2 i \epsilon}\right)\right],
\end{aligned}
$$

where $K$ and $E$ are complete elliptic integrals of the first and second kind, respectively. Inserting this result into Eq. (D16) yields

$$
\begin{aligned}
A^{(1)}\left(\omega ; x_{3}, x_{0}\right) & =\lim _{\epsilon \rightarrow 0^{+}}-\frac{i e^{-i \pi h_{\sigma}} e^{i \omega\left(x_{3}-x_{0}-L_{a}\right)}}{2 \pi} \int_{-\infty}^{\infty} d t_{2} e^{i \omega t_{2}} \frac{2 i^{1 / 8}}{L_{a}^{1 / 8}}\left[K\left(\frac{L_{a}}{t_{2}-L_{a}-2 i \epsilon}\right)-E\left(\frac{L_{a}}{t_{2}-L_{a}-2 i \epsilon}\right)\right] \\
& =\lim _{\epsilon^{\prime} \rightarrow 0^{+}} 2 i L_{a}^{7 / 8} e^{i \omega\left(x_{3}-x_{0}\right)} \int_{-\infty}^{\infty} \frac{d y}{2 \pi} e^{i\left(\omega L_{a}\right) y}\left[E\left(\frac{1}{y-i \epsilon^{\prime}}\right)-K\left(\frac{1}{y-i \epsilon^{\prime}}\right)\right] .
\end{aligned}
$$


From the first to the second line, we substitute $t_{2}=$ $L_{a}(y+1)$ and introduce $\epsilon^{\prime} \propto \epsilon$ as the small parameter to be taken to zero. Finally, we write the amplitude as

$$
A^{(1)}\left(\omega ; x_{3}, x_{0}\right)=2 i L_{a}^{7 / 8} e^{i \omega\left(x_{3}-x_{0}\right)} g\left(\omega L_{a}\right),
$$

where $g(u)$ is defined as a Fourier transform via

$$
\begin{gathered}
g(u)=\lim _{\epsilon \rightarrow 0^{+}} \int_{-\infty}^{\infty} \frac{d y}{2 \pi} e^{i u y} \tilde{g}(y-i \epsilon), \\
\tilde{g}(y)=E\left(\frac{1}{y}\right)-K\left(\frac{1}{y}\right) .
\end{gathered}
$$

States $\left|\psi_{\omega}\right\rangle$ with negative frequencies do not exist in a chiral CFT, so strictly speaking, the amplitude is ill-defined for $\omega<0$, and hence, $g(u<0)$ is ill-defined as well. Nevertheless, it is convenient to now extend the domain of $g(u)$ defined in Eq. (D23a) to all real $u$. [We caution that one should not confuse this continuation with that adopted later in Eq. (F6), which serves a quite different purpose.] The function $g(u)$ extended in this way vanishes for $u<0$, since $\tilde{g}(y)$ has no singularities in the lower half plane $(\operatorname{Im} y<0)$. In addition, $\lim _{\epsilon \rightarrow 0^{+}} \operatorname{Re} \tilde{g}(y-i \epsilon)$ is symmetric in $y$, while $\lim _{\epsilon \rightarrow 0^{+}} \operatorname{Im} \tilde{g}(y-i \epsilon)$ is antisymmetric. Together, these properties allow us to write $g(u)$ over the physical domain $u \geq 0$ in terms of simply the imaginary part of $\tilde{g}$ :

$$
\begin{gathered}
g(u \geq 0)=\frac{2}{\pi} \int_{0}^{\infty} d y \sin (u y) \tilde{g}_{i}(y), \\
\tilde{g}_{i}(y)=-\lim _{\epsilon \rightarrow 0^{+}} \operatorname{Im} \tilde{g}(y-i \epsilon) .
\end{gathered}
$$

For $y>1$ or $y<-1, \tilde{g}(y)$ is purely real because $1 / y$ lies within the interval $-1<(1 / y)<1$ (cf. Appendix E). Using Eqs. (E2) and (E3), the imaginary component $\tilde{g}_{i}$ can be written as

$$
\tilde{g}_{i}(y)= \begin{cases}0, & |y|>1, \\ \frac{1}{y} E\left(\sqrt{1-y^{2}}\right), & |y| \leq 1,\end{cases}
$$

which is supported on the finite interval $-1 \leq y \leq 1$. From Eqs. D 3 we can now reexpress $g(u \geq 0)$ as

$$
g(u \geq 0)=\frac{2}{\pi} \int_{0}^{1} d y \frac{\sin (u y)}{y} E\left(\sqrt{1-y^{2}}\right) .
$$

Evidently, $g$ is a purely real function. As we show in Appendix E 2, it can be written in terms of a generalized hypergeometric function,

$$
g(u \geq 0)=\frac{\pi u}{4}{ }_{1} F_{2}\left(\frac{1}{2} ; 1,2 \mid-\frac{1}{4} u^{2}\right)
$$

plotted in Fig. 11(a).

Upon replacing $\omega \rightarrow k_{e}$ to match the notation from Sec. VII A and setting $x_{3}-x_{0}=L_{e}$, the zeroth- and first-order terms in the transmission amplitude are

$$
\begin{gathered}
A^{(0)}\left(k_{e}\right)=e^{i k_{e} L_{e}}, \\
t_{\sigma} A^{(1)}\left(k_{e} ; x_{3}, x_{0}\right)=e^{i k_{e} L_{e}} \times 2 i t_{\sigma} L_{a}^{7 / 8} g\left(k_{e} L_{a}\right) .
\end{gathered}
$$

Restoring $v_{e}$ factors recovers precisely the $t_{\sigma}$ correction quoted in Eqs. (46) and (48) in the $n_{\psi}=0$ case.

\section{APPENDIX E: A FEW FACTS REGARDING COMPLETE ELLIPTIC INTEGRALS}

Let $K(k)$ and $E(k)$ denote the complete elliptic integrals of the first and second kind, respectively. For $|k|<1$, they are defined as

$$
\begin{gathered}
K(k)=\frac{1}{4} \int_{0}^{2 \pi} \frac{d \theta}{\sqrt{1-k^{2} \cos ^{2} \theta}}, \\
E(k)=\frac{1}{4} \int_{0}^{2 \pi} d \theta \sqrt{1-k^{2} \cos ^{2} \theta}
\end{gathered}
$$

beyond the unit circle they are defined via analytical continuation. Both are even [e.g., $K(-k)=K(k)$ ] and have branch cuts along the real line at $k \leq-1$ and $k \geq 1$.

Define $\bar{x}=\sqrt{1-x^{2}}$. For $0<x<1$, these functions satisfy the algebraic identities [134-137]

$$
\lim _{\epsilon \rightarrow 0^{+}} \frac{K\left(\frac{1}{x}+i \epsilon\right)-K\left(\frac{1}{x}-i \epsilon\right)}{2}=i x K(\bar{x}),
$$

$\lim _{\epsilon \rightarrow 0^{+}} \frac{E\left(\frac{1}{x}+i \epsilon\right)-E\left(\frac{1}{x}-i \epsilon\right)}{2}=i\left[x K(\bar{x})-\frac{1}{x} E(\bar{x})\right]$

along with the differential and integral identities

$$
\begin{gathered}
\frac{d}{d x} E(\bar{x})=\frac{x}{1-x^{2}}[K(\bar{x})-E(\bar{x})], \\
\frac{d}{d x}[E(\bar{x})-K(\bar{x})]=\frac{1}{x} E(\bar{x}), \\
\int_{0}^{1} d x E(\bar{x})=\int_{0}^{1} d x \frac{x}{\bar{x}} E(x)=\frac{\pi^{2}}{8} .
\end{gathered}
$$

\section{An integral identity}

Suppose that $a, b$ are complex numbers such that $\operatorname{Re} a>0$ and $\operatorname{Re} b>0$. Following hours of struggle with Mathematica, one can show that 


$$
\begin{aligned}
\int_{-\infty}^{\infty} d z\left[\sqrt{\frac{z^{2}+a^{2}}{z^{2}+b^{2}}}+\sqrt{\frac{z^{2}+b^{2}}{z^{2}+a^{2}}}-2\right] \\
=4(a+b)\left[K\left(\frac{a-b}{a+b}\right)-E\left(\frac{a-b}{a+b}\right)\right] .
\end{aligned}
$$

To be precise, the integrand has branch points at $\pm i a$ and $\pm i b$; the integral is evaluated assuming branch cuts between $i a \leftrightarrow i b$ and $-i a \leftrightarrow-i b$ with one pair above the real line and the other pair below.

\section{A different integral identity}

Here we show that the integral

$$
g(u) \stackrel{\text { def }}{=} \frac{2}{\pi} \int_{0}^{1} d y \frac{\sin (u y)}{y} E\left(\sqrt{1-y^{2}}\right)
$$

can be expressed as a generalized hypergeometric function

$$
\begin{aligned}
g(u) & \stackrel{?}{=} \frac{\pi u}{4}{ }_{1} F_{2}\left(\frac{1}{2} ; 1,2 \mid-\frac{1}{4} u^{2}\right) \\
& =\frac{\pi u}{4} \sum_{m=0}^{\infty} \frac{1}{m !} \frac{\left(\frac{1}{2}\right)\left(\frac{3}{2}\right) \cdots\left(\frac{2 m-1}{2}\right)}{m !(m+1) !}\left(-\frac{1}{4} u^{2}\right)^{m} \\
& =\frac{\pi u}{4} \sum_{m=0}^{\infty} \frac{(-1)^{m}(2 m-1) ! !}{4^{m} m !(m+1) !(2 m) ! !} u^{2 m}
\end{aligned}
$$

To do so, we Taylor expand Eq. (E6) in powers of $u$ and show that it takes the form of Eq. (E7).

Notice that terms with even powers vanish in Eq. (E6). The coefficients for odd powers $u^{2 m+1}$ are given by

$$
c_{2 m+1}=\frac{2}{\pi} \frac{(-1)^{m}}{(2 m+1) !} M_{m}
$$

with

$$
M_{m}=\int_{0}^{1} d y y^{2 m} E\left(\sqrt{1-y^{2}}\right) .
$$

One can evaluate $M_{m}$ as follows. Performing integration by parts twice and using Eqs. (E4a) and (E4b) yields $M_{m}=$ $(2 m+1)^{2} M_{m}-\left(4 m^{2}-1\right) M_{m-1}$, implying the recursion relation

$$
M_{m}=\frac{(2 m+1)(2 m-1)}{4 m(m+1)} M_{m-1} .
$$

Since $M_{0}=\left(\pi^{2} / 8\right)$ from Eq. (E4c), we can deduce that $4^{m} m !(m+1) ! M_{m}=\left(\pi^{2} / 8\right)(2 m-1) ! !(2 m+1) ! !$. Therefore,

$$
c_{2 m+1}=\frac{\pi}{4}(-1)^{m} \frac{(2 m-1) ! !}{4^{m} m !(m+1) !(2 m) ! !},
$$

which indeed matches the coefficients in the series expansion in Eq. (E7).

\section{APPENDIX F: EXTRACTION OF ISING-ANYON TUNNELING WEIGHTS}

Here we formally invert Eq. (47) to extract the scaling functions $f_{i v}$ and $f_{v}$ that quantify energy partitioning in Ising-anyon tunneling events. First we define

$$
G(u)=2 i g(u) u^{7 / 8}
$$

and

$$
F(y)= \begin{cases}f_{v}(y), & y>0 \\ f_{i v}(-y), & -1<y<0 \\ 0 & y<-1,\end{cases}
$$

so that Eq. (47) can be compactly expressed as

$$
G(u)=\int_{-1}^{\infty} d y e^{i u y} F(y) .
$$

Since $G(u)$ is defined only for $u>0$, one cannot exploit standard plane-wave orthogonality to isolate $F(y)$. To proceed, we continue $G(u)$ to $u<0$. For clarity, we denote the resulting function defined for all real $u$ by $G_{c}(u)$.

Care must be taken in defining this continuation to ensure that $F(y<-1)$ remains zero as demanded by our physical system. Consider the integral

$$
\mathcal{F}(y) \equiv \int_{0}^{\infty} \frac{d u}{2 \pi} e^{-i u y} e^{-\epsilon u} G(u),
$$

where we introduce a regulator with $\epsilon \rightarrow 0^{+}$for convergence, which is necessary given that $G(u) \sim u^{7 / 8}$ as $u \rightarrow \infty$. Evaluating this integral gives a result in terms of a generalized hypergeometric function. Here we simply note that

$$
\begin{gathered}
|\mathcal{F}(y)|=|\mathcal{F}(-y)|, \\
\mathcal{F}(y>1)=-e^{i(\pi / 16)}|\mathcal{F}(y>1)|, \\
\mathcal{F}(y<-1)=e^{-i(\pi / 16)}|\mathcal{F}(y<-1)| .
\end{gathered}
$$

These crucial properties imply that the desired continuation is given by

$$
G_{c}(u)= \begin{cases}G(u), & u>0, \\ e^{-i(\pi / 8)} G(|u|), & u<0 .\end{cases}
$$

It follows that 
$\int_{-\infty}^{\infty} \frac{d u}{2 \pi} e^{-i u y} e^{-\epsilon|u|} G_{c}(u)=\mathcal{F}(y)+e^{-i(\pi / 8)} \mathcal{F}(-y)=F(y)$,

where again we introduce a regulator with $\epsilon \rightarrow 0^{+}$. By virtue of Eqs. (F5a) through (F5c), we see that $F(y<-1)$ indeed vanishes. Taking the $\epsilon \rightarrow 0^{+}$limit allows us to explicitly deduce

$$
\begin{aligned}
& e^{i(9 \pi / 16)} f_{i v}(y) \\
& =\frac{2 \Gamma\left(\frac{15}{8}\right)}{\pi y^{15 / 8} 3} F_{2}\left(-\frac{1}{2}, \frac{1}{2}, \frac{1}{2} ;-\frac{7}{16}, \frac{1}{16} \mid y^{2}\right) \\
& -\frac{7^{2} \sqrt{\pi} \Gamma\left(\frac{1}{16}\right)}{2^{25 / 8} \times 15 \Gamma\left(\frac{9}{16}\right)^{3}} F_{2}\left(\frac{7}{16}, \frac{23}{16}, \frac{23}{16} ; \frac{1}{2}, \frac{31}{16} \mid y^{2}\right) \\
& +\frac{2^{15 / 8} \times 15^{2} \sqrt{\pi} \Gamma\left(\frac{9}{16}\right) y}{7 \times 23 \Gamma\left(\frac{1}{16}\right)^{3}}{ }_{3} F_{2}\left(\frac{15}{16}, \frac{31}{16}, \frac{31}{16} ; \frac{3}{2}, \frac{39}{16} \mid y^{2}\right)
\end{aligned}
$$

and

$$
\begin{aligned}
& e^{i(9 \pi / 16)} f_{v}(y<1) \\
& =\frac{2 \cos \left(\frac{\pi}{8}\right) \Gamma\left(\frac{15}{8}\right)}{\pi y^{15 / 8}}{ }_{3} F_{2}\left(-\frac{1}{2}, \frac{1}{2}, \frac{1}{2} ;-\frac{7}{16}, \frac{1}{16} \mid y^{2}\right) \\
& \quad-\frac{7^{2} \sqrt{\pi} \Gamma\left(\frac{1}{16}\right)}{2^{25 / 8} \times 15 \Gamma\left(\frac{9}{16}\right)^{3}} F_{2}\left(\frac{7}{16}, \frac{23}{16}, \frac{23}{16} ; \frac{1}{2}, \frac{31}{16} \mid y^{2}\right) \\
& \quad+\frac{2^{15 / 8} \times 15^{2} \sqrt{\pi} \Gamma\left(\frac{9}{16}\right) y}{7 \times 23 \Gamma\left(\frac{1}{16}\right)^{3}}{ }_{3} F_{2}\left(\frac{15}{16}, \frac{31}{16}, \frac{31}{16} ; \frac{3}{2}, \frac{39}{16} \mid y^{2}\right), \\
& e^{i(9 \pi / 16)} f_{v}(y>1)=\frac{\sin \left(\frac{\pi}{8}\right) \Gamma\left(\frac{23}{8}\right)}{2 y^{23 / 8}}{ }_{3} F_{2}\left(\frac{1}{2}, \frac{23}{16}, \frac{31}{16} ; 1, \mid \frac{1}{y^{2}}\right) .
\end{aligned}
$$

[Recall that $f_{i v}(y)$ is defined for $0<y<1$, while $f_{v}(y)$ is defined for $y>0$.] We thus complete the desired inversion. Figure 11(b) from the main text plots the magnitude of the scaling functions $f_{i v}$ and $f_{v}$.

Some limits of $F(y)$ can be deduced from the asymptotics specified in Eqs. (50) and (52). The small-y singularities follow from the leading $G(u \gg 1)$ behavior and can be obtained by simply replacing $G(u) \rightarrow 2 i u^{7 / 8}$ in Eq. (F7). One finds

$$
\begin{aligned}
F(|y| \ll 1) & \approx \frac{\Gamma\left(\frac{15}{8}\right)}{\pi}\left[\frac{e^{i(\pi / 2)}}{(\epsilon+i y)^{15 / 8}}+\frac{e^{i(3 \pi / 8)}}{(\epsilon-i y)^{15 / 8}}\right] \\
& \approx \begin{cases}-i e^{-i(\pi / 16)}\left[\frac{2}{\pi} \cos \left(\frac{\pi}{8}\right) \Gamma\left(\frac{15}{8}\right)\right] y^{-15 / 8}, & y>0, \\
-i e^{-i(\pi / 16)}\left[\frac{2}{\pi} \Gamma\left(\frac{15}{8}\right)\right]|y|^{-15 / 8}, & y<0 .\end{cases}
\end{aligned}
$$

In the bottom lines, we take the $\epsilon \rightarrow 0^{+}$limit. We caution, however, that inserting the bottom lines of Eq. (F11) into Eq. (F3) would produce an unphysical infrared divergence; hence, in the top line we explicitly display the regularization that circumvents this problem. Elsewhere, we are free to send $\epsilon \rightarrow 0^{+}$as no such issues arise. The singularities at $y \rightarrow \pm 1^{+}$instead follow from the subleading $G(u \gg 1)$ behavior; they can be captured by replacing $G(u) \rightarrow$ $-2 i u^{-1 / 8}(\cos u)$ in Eq. (F7), yielding

$$
F\left(y \rightarrow \pm 1^{+}\right) \approx-i e^{-i(\pi / 16)}\left[\frac{1}{\Gamma\left(\frac{1}{8}\right)}\right](y \mp 1)^{-7 / 8} .
$$

Finally, the asymptotic decay at $y \gg 1$ encodes the $G(u \ll 1)$ behavior and follows from replacing $G(u) \rightarrow$ $i(\pi / 2) u^{15 / 8}$ in Eq. (F3):

$$
F(y \gg 1) \approx-i e^{-i(\pi / 16)}\left[\frac{\pi}{2 \Gamma\left(-\frac{15}{8}\right)}\right] y^{-23 / 8} .
$$

Figure 11(b) indicates the scaling behaviors captured above.

\section{APPENDIX G: CONDUCTANCE FROM MAJORANA PHASE ACCUMULATION}

For completeness, we briefly review how electrical conductance follows from the relative phases acquired by Majorana fermions propagating in the circuits from Figs. 9 and 10. We denote the part of the wave function describing an electron incident at the lower edge of the $\nu=1$ quantum-Hall system by

$$
\int_{-\infty}^{x_{0}} d x e^{i E x / u} c^{\dagger}(x)|0\rangle
$$

Here, $|0\rangle$ is the ground state, $E$ is the incident energy, $u$ is the edge velocity in the region without induced pairing, $c^{\dagger}(x)$ adds the electron to position $x$ of the edge, and $x_{0}$ is the location at which the edge state meets the proximitizing superconductor. (In this Appendix, we use coordinates consistent with those in Fig. 17.) Employing a Majorana representation via $c=\gamma_{1}+i \gamma_{2}$, Eq. (G1) equivalently becomes

$$
\int_{-\infty}^{x_{0}} d x e^{i E x / u}\left[\gamma_{1}(x)-i \gamma_{2}(x)\right]|0\rangle
$$

Beyond position $x_{0}$, the constituent Majorana fermions $\gamma_{1}$ and $\gamma_{2}$ follow diverging paths that eventually recombine at position $x_{3}$ at the upper edge of the $\nu=1$ quantum-Hall system. En route, they generally acquire different phase factors; hence, at the upper $\nu=1$ edge, the outgoing part of the wave function becomes 


$$
e^{i \bar{\phi}(E)} \int_{x_{3}}^{-\infty} d x e^{i E x / u}\left[\gamma_{1}(x)-i e^{i \delta \phi(E)} \gamma_{2}(x)\right]|0\rangle,
$$

where $\bar{\phi}(E)$ denotes a possible phase common to both Majorana fermions (which is unimportant here) and $\delta \phi(E)$ is the accumulated phase difference. Reverting back to complex fermions by writing $\gamma_{1}=\left(c+c^{\dagger}\right) / 2$ and $\gamma_{2}=-i\left(c-c^{\dagger}\right) / 2$, Eq. (G3) reads

$$
\begin{aligned}
e^{i \bar{\phi}(E)} \int_{x_{3}}^{-\infty} d x e^{i E x / u}\left[\left(\frac{1+e^{i \delta \phi(E)}}{2}\right) c^{\dagger}(x)\right. \\
\left.+\left(\frac{1-e^{i \delta \phi(E)}}{2}\right) c(x)\right]|0\rangle .
\end{aligned}
$$

Thus, with probability

$$
P_{A}(E)=\left|\frac{1-e^{i \delta \phi(E)}}{2}\right|^{2}=\frac{1-\cos [\delta \phi(E)]}{2},
$$

the incident electron returns at the upper edge as a holetransmitting a Cooper pair into the superconductor. The conductance at bias voltage $V$ arising from such Andreev processes is

$$
G(V)=\frac{2 e^{2}}{h} P_{A}(e V)
$$

[1] A. Yu. Kitaev, Fault-Tolerant Quantum Computation by Anyons, Ann. Phys. (Amsterdam) 303, 2 (2003).

[2] C. Nayak, S. H. Simon, A. Stern, M. Freedman, and S. D. Sarma, Non-Abelian Anyons and Topological Quantum Computation, Rev. Mod. Phys. 80, 1083 (2008).

[3] R. Willett, J. P. Eisenstein, H. L. Störmer, D. C. Tsui, A. C. Gossard, and J.H. English, Observation of an EvenDenominator Quantum Number in the Fractional Quantum Hall Effect, Phys. Rev. Lett. 59, 1776 (1987).

[4] G. Moore and N. Read, Nonabelions in the Fractional Quantum Hall Effect, Nucl. Phys. B360, 362 (1991).

[5] M. Levin, B. I. Halperin, and B. Rosenow, Particle-Hole Symmetry and the Pfaffian State, Phys. Rev. Lett. 99, 236806 (2007).

[6] S.-S. Lee, S. Ryu, C. Nayak, and M. P. A. Fisher, ParticleHole Symmetry and the $\nu=\frac{5}{2}$ Quantum Hall State, Phys. Rev. Lett. 99, 236807 (2007).

[7] D. T. Son, Is the Composite Fermion a Dirac Particle?, Phys. Rev. X 5, 031027 (2015).

[8] M. Banerjee, M. Heiblum, V. Umansky, D. E. Feldman, Y. Oreg, and A. Stern, Observation of Half-Integer Thermal Hall Conductance, Nature (London) 559, 205 (2018).

[9] S. D. Sarma, M. Freedman, and C. Nayak, Topologically Protected Qubits from a Possible Non-Abelian Fractional Quantum Hall State, Phys. Rev. Lett. 94, 166802 (2005).
[10] A. Stern and B.I. Halperin, Proposed Experiments to Probe the Non-Abelian $\nu=5 / 2$ Quantum Hall State, Phys. Rev. Lett. 96, 016802 (2006).

[11] P. Bonderson, A. Kitaev, and K. Shtengel, Detecting NonAbelian Statistics in the $\nu=5 / 2$ Fractional Quantum Hall State, Phys. Rev. Lett. 96, 016803 (2006).

[12] R. L. Willett, K. Shtengel, C. Nayak, L. N. Pfeiffer, Y. J. Chung, M. L. Peabody, K. W. Baldwin, and K. W. West, Interference Measurements of Non-Abelian e/4 and Abelian e/2 Quasiparticle Braiding, arXiv:1905.10248.

[13] N. Read and D. Green, Paired States of Fermions in Two Dimensions with Breaking of Parity and Time-Reversal Symmetries and the Fractional Quantum Hall Effect, Phys. Rev. B 61, 10267 (2000).

[14] A. Yu. Kitaev, Unpaired Majorana Fermions in Quantum Wires, Sov. Phys. Usp. 44, 131 (2001).

[15] M. Z. Hasan and C. L. Kane, Colloquium: Topological Insulators, Rev. Mod. Phys. 82, 3045 (2010).

[16] X.-L. Qi and S.-C. Zhang, Topological Insulators and Superconductors, Rev. Mod. Phys. 83, 1057 (2011).

[17] C. W. J. Beenakker, Search for Majorana Fermions in Superconductors, Annu. Rev. Condens. Matter Phys. 4, 113 (2013).

[18] J. Alicea, New Directions in the Pursuit of Majorana Fermions in Solid State Systems, Rep. Prog. Phys. 75, 076501 (2012).

[19] M. Leijnse and K. Flensberg, Introduction to Topological Superconductivity and Majorana Fermions, Semicond. Sci. Technol. 27, 124003 (2012).

[20] T. D. Stanescu and S. Tewari, Majorana Fermions in Semiconductor Nanowires: Fundamentals, Modeling, and Experiment, J. Phys. Condens. Matter 25, 233201 (2013).

[21] S. R. Elliott and M. Franz, Colloquium: Majorana Fermions in Nuclear, Particle, and Solid-State Physics, Rev. Mod. Phys. 87, 137 (2015).

[22] S. D. Sarma, M. Freedman, and C. Nayak, Majorana Zero Modes and Topological Quantum Computation, npj Quantum Inf. 1, 15001 (2015).

[23] M. Sato and S. Fujimoto, Majorana Fermions and Topology in Superconductors, J. Phys. Soc. Jpn. 85, 072001 (2016).

[24] R. Aguado, Majorana Quasiparticles in Condensed Matter, Riv. Nuovo Cimento 40, 523 (2017).

[25] R. M. Lutchyn, E. P. A. M. Bakkers, L. P. Kouwenhoven, P. Krogstrup, C. M. Marcus, and Y. Oreg, Majorana Zero Modes in Superconductor/Semiconductor Heterostructures, Nat. Rev. Mater. 3, 52 (2018).

[26] L. Fu and C. L. Kane, Probing Neutral Majorana Fermion Edge Modes with Charge Transport, Phys. Rev. Lett. 102, 216403 (2009).

[27] A. R. Akhmerov, J. Nilsson, and C. W. J. Beenakker, Electrically Detected Interferometry of Majorana Fermions in a Topological Insulator, Phys. Rev. Lett. 102, 216404 (2009).

[28] K. T. Law, P. A. Lee, and T. K. Ng, Majorana Fermion Induced Resonant Andreev Reflection, Phys. Rev. Lett. 103, 237001 (2009).

[29] L. Fu, Electron Teleportation via Majorana Bound States in a Mesoscopic Superconductor, Phys. Rev. Lett. 104, 056402 (2010). 
[30] S. B. Chung, X.-L. Qi, J. Maciejko, and S.-C. Zhang, Conductance and Noise Signatures of Majorana Backscattering, Phys. Rev. B 83, 100512(R) (2011).

[31] T. Karzig, C. Knapp, R. M. Lutchyn, P. Bonderson, M. B. Hastings, C. Nayak, J. Alicea, K. Flensberg, S. Plugge, Y. Oreg, C. M. Marcus, and M.H. Freedman, Scalable Designs for Quasiparticle-Poisoning-Protected Topological Quantum Computation with Majorana Zero Modes, Phys. Rev. B 95, 235305 (2017).

[32] K. W. Plumb, J. P. Clancy, L. J. Sandilands, V. V. Shankar, Y. F. Hu, K. S. Burch, H.-Y. Kee, and Y.-J. Kim, $\alpha-\mathrm{RuCl}_{3}$ : A Spin-Orbit Assisted Mott Insulator on a Honeycomb Lattice, Phys. Rev. B 90, 041112(R) (2014).

[33] H.-S. Kim, V. V. Shankar, A. Catuneanu, and H.-Y. Kee, Kitaev Magnetism in Honeycomb $\alpha-\mathrm{RuCl}_{3}$ with Intermediate Spin-Orbit Coupling, Phys. Rev. B 91, 241110 (2015).

[34] A. Kitaev, Anyons in an Exactly Solved Model and Beyond, Ann. Phys. (Amsterdam) 321, 2 (2006).

[35] G. Jackeli and G. Khaliullin, Mott Insulators in the Strong Spin-Orbit Coupling Limit: From Heisenberg to a Quantum Compass and Kitaev Models, Phys. Rev. Lett. 102, 017205 (2009).

[36] S. M. Winter, A. A. Tsirlin, M. Daghofer, J. van den Brink, Y. Singh, P. Gegenwart, and R. Valentí, Models and Materials for Generalized Kitaev Magnetism, J. Phys. Condens. Matter 29, 493002 (2017).

[37] S. Trebst, Kitaev Materials, arXiv:1701.07056.

[38] L. Janssen and M. Vojta, Heisenberg-Kitaev Physics in Magnetic Fields, J. Phys. Condens. Matter 31, 423002 (2019).

[39] Y. Motome and J. Nasu, Hunting Majorana Fermions in Kitaev Magnets, J. Phys. Soc. Jpn. 89, 012002 (2020).

[40] J. A. Sears, M. Songvilay, K. W. Plumb, J. P. Clancy, Y. Qiu, Y. Zhao, D. Parshall, and Y.-J. Kim, Magnetic Order in $\alpha-\mathrm{RuCl}_{3}:$ A Honeycomb-Lattice Quantum Magnet with Strong Spin-Orbit Coupling, Phys. Rev. B 91, 144420 (2015).

[41] J. Chaloupka, G. Jackeli, and G. Khaliullin, Zigzag Magnetic Order in the Iridium Oxide $\mathrm{Na}_{2} \mathrm{IrO}_{3}$, Phys. Rev. Lett. 110, 097204 (2013).

[42] L. J. Sandilands, Y. Tian, K. W. Plumb, Y.-J. Kim, and K.S. Burch, Scattering Continuum and Possible Fractionalized Excitations in $\alpha-\mathrm{RuCl}_{3}$, Phys. Rev. Lett. 114, 147201 (2015).

[43] J. Nasu, J. Knolle, D. L. Kovrizhin, Y. Motome, and R. Moessner, Fermionic Response from Fractionalization in an Insulating Two-Dimensional Magnet, Nat. Phys. 12, 912 (2016).

[44] A. Banerjee, C. A. Bridges, J.-Q. Yan, A. A. Aczel, L. Li, M. B. Stone, G. E. Granroth, M. D. Lumsden, Y. Yiu, J. Knolle, S. Bhattacharjee, D. L. Kovrizhin, R. Moessner, D. A. Tennant, D. G. Mandrus, and S. E. Nagler, Proximate Kitaev Quantum Spin Liquid Behaviour in a Honeycomb Magnet, Nat. Mater. 15, 733 (2016).

[45] A. Banerjee, J. Yan, J. Knolle, C. A. Bridges, M. B. Stone, M. D. Lumsden, D. G. Mandrus, D. A. Tennant, R. Moessner, and S. E. Nagler, Neutron Scattering in the Proximate Quantum Spin Liquid $\alpha-\mathrm{RuCl}_{3}$, Science 356, 1055 (2017).

[46] S.-H. Do, S.-Y. Park, J. Yoshitake, J. Nasu, Y. Motome, Y.S. Kwon, D. T. Adroja, D. J. Voneshen, K. Kim,
T.-H. Jang, J.-H. Park, K.-Y. Choi, and S. Ji, Majorana Fermions in the Kitaev Quantum Spin System $\alpha-\mathrm{RuCl}_{3}$, Nat. Phys. 13, 1079 (2017).

[47] Y. Kasahara, K. Sugii, T. Ohnishi, M. Shimozawa, M. Yamashita, N. Kurita, H. Tanaka, J. Nasu, Y. Motome, T. Shibauchi, and Y. Matsuda, Unusual Thermal Hall Effect in a Kitaev Spin Liquid Candidate $\alpha-\mathrm{RuCl}_{3}$, Phys. Rev. Lett. 120, 217205 (2018).

[48] C. Wellm, J. Zeisner, A. Alfonsov, A. U. B. Wolter, M. Roslova, A. Isaeva, T. Doert, M. Vojta, B. Büchner, and V. Kataev, Signatures of Low-Energy Fractionalized Excitations in $\alpha-\mathrm{RuCl}_{3}$ from Field-Dependent Microwave Absorption, Phys. Rev. B 98, 184408 (2018).

[49] Y. Wang, G. B. Osterhoudt, Y. Tian, P. Lampen-Kelley, A. Banerjee, T. Goldstein, J. Yan, J. Knolle, H. Ji, R. J. Cava, J. Nasu, Y. Motome, S. E. Nagler, D. Mandrus, and K. S. Burch, Direct Evidence for Fermi Statistics from Proximity to the Kitaev Spin Liquid in $\mathrm{RuCl}_{3}$, arXiv:1809.07782.

[50] N. Jansa, A. Zorko, M. Gomilsek, M. Pregelj, K. W. Kramer, D. Biner, A. Biffin, C. Ruegg, and M. Klanjsek, Observation of Two Types of Fractional Excitation in the Kitaev Honeycomb Magnet, Nat. Phys. 14, 786 (2018).

[51] S. Widmann, V. Tsurkan, D. A. Prishchenko, V. G. Mazurenko, A. A. Tsirlin, and A. Loidl, Thermodynamic Evidence of Fractionalized Excitations in $\alpha-\mathrm{RuCl}_{3}$, Phys. Rev. B 99, 094415 (2019).

[52] H. Zhang, S. Kim, Y.-J. Kim, H.-Y. Kee, and L. Yang, Ultrafast Dynamics of Fractional Particles in $\alpha-\mathrm{RuCl}_{3}$, arXiv:1908.04807.

[53] R. D. Johnson, S. C. Williams, A. A. Haghighirad, J. Singleton, V. Zapf, P. Manuel, I. I. Mazin, Y. Li, H. O. Jeschke, R. Valentí, and R. Coldea, Monoclinic Crystal Structure of $\alpha-\mathrm{RuCl}_{3}$ and the Zigzag Antiferromagnetic Ground State, Phys. Rev. B 92, 235119 (2015).

[54] S.-H. Baek, S.-H. Do, K.-Y. Choi, Y. S. Kwon, A. U. B. Wolter, S. Nishimoto, J. van den Brink, and B. Büchner, Evidence for a Field-Induced Quantum Spin Liquid in $\alpha-\mathrm{RuCl}_{3}$, Phys. Rev. Lett. 119, 037201 (2017).

[55] J. A. Sears, Y. Zhao, Z. Xu, J. W. Lynn, and Y.-J. Kim, Phase Diagram of $\alpha-\mathrm{RuCl}_{3}$ in an In-Plane Magnetic Field, Phys. Rev. B 95, 180411(R) (2017).

[56] A. U. B. Wolter, L. T. Corredor, L. Janssen, K. Nenkov, S. Schönecker, S.-H. Do, K.-Y. Choi, R. Albrecht, J. Hunger, T. Doert, M. Vojta, and B. Büchner, Field-Induced Quantum Criticality in the Kitaev System $\alpha-\mathrm{RuCl}_{3}$, Phys. Rev. B 96, 041405(R) (2017).

[57] I. A. Leahy, C. A. Pocs, P. E. Siegfried, D. Graf, S.-H. Do, K.-Y. Choi, B. Normand, and M. Lee, Anomalous Thermal Conductivity and Magnetic Torque Response in the Honeycomb Magnet $\alpha-\mathrm{RuCl}_{3}$, Phys. Rev. Lett. 118, 187203 (2017).

[58] A. Banerjee, P. Lampen-Kelley, J. Knolle, C. Balz, A. A. Aczel, B. Winn, Y. Liu, D. Pajerowski, J. Yan, C. A. Bridges, A. T. Savici, B. C. Chakoumakos, M. D. Lumsden, D. A. Tennant, R. Moessner, D. G. Mandrus, and S. E. Nagler, Excitations in the Field-Induced Quantum Spin Liquid State of $\alpha-\mathrm{RuCl}_{3}$, npj Quantum Mater. 3, 8 (2018).

[59] R. Hentrich, A. U. B. Wolter, X. Zotos, W. Brenig, D. Nowak, A. Isaeva, T. Doert, A. Banerjee, P. Lampen-Kelley, 
D. G. Mandrus, S. E. Nagler, J. Sears, Y.-J. Kim, B. Büchner, and C. Hess, Unusual Phonon Heat Transport in $\alpha-\mathrm{RuCl}_{3}$ : Strong Spin-Phonon Scattering and Field-Induced Spin Gap, Phys. Rev. Lett. 120, 117204 (2018).

[60] Y. Kasahara, T. Ohnishi, Y. Mizukami, O. Tanaka, S. Ma, K. Sugii, N. Kurita, H. Tanaka, J. Nasu, Y. Motome, T. Shibauchi, and Y. Matsuda, Majorana Quantization and Half-Integer Thermal Quantum Hall Effect in a Kitaev Spin Liquid, Nature (London) 559, 227 (2018).

[61] C. Balz, P. Lampen-Kelley, A. Banerjee, J. Yan, Z. Lu, X. Hu, S. M. Yadav, Y. Takano, Y. Liu, D. A. Tennant, M. D. Lumsden, D. Mandrus, and S. E. Nagler, Finite Field Regime for a Quantum Spin Liquid in $\alpha-\mathrm{RuCl}_{3}$, Phys. Rev. B 100, 060405(R) (2019).

[62] M. Ye, G. B. Halász, L. Savary, and L. Balents, Quantization of the Thermal Hall Conductivity at Small Hall Angles, Phys. Rev. Lett. 121, 147201 (2018).

[63] Y. Vinkler-Aviv and A. Rosch, Approximately Quantized Thermal Hall Effect of Chiral Liquids Coupled to Phonons, Phys. Rev. X 8, 031032 (2018).

[64] T. Yokoi, S. Ma, Y. Kasahara, S. Kasahara, T. Shibauchi, N. Kurita, H. Tanaka, J. Nasu, Y. Motome, C. Hickey, S. Trebst, and Y. Matsuda, Half-Integer Quantized Anomalous Thermal Hall Effect in the Kitaev Material $\alpha-\mathrm{RuCl}_{3}$, arXiv:2001.01899.

[65] Any topological-quantum-computing platform would ideally be run at the lowest accessible temperatures. A large gap is nevertheless desirable for suppressing errors.

[66] B. Zhou, J. Balgley, P. Lampen-Kelley, J.-Q. Yan, D. G. Mandrus, and E.A. Henriksen, Gate-Tuned ChargeDoping and Magnetism in Graphene $/ \alpha-\mathrm{RuCl}_{3}$ Heterostructures, Phys. Rev. B 100, 165426 (2019).

[67] B. Zhou, Y. Wang, G. B. Osterhoudt, P. Lampen-Kelley, D. Mandrus, R. He, K.S. Burch, and E. A. Henriksen, Possible Structural Transformation and Enhanced Magnetic Fluctuations in Exfoliated $\alpha-\mathrm{RuCl}_{3}$, J. Phys. Chem. Solids 128, 291 (2019).

[68] S. Mashhadi, D. Weber, L. M. Schoop, A. Schulz, B. V. Lotsch, M. Burghard, and K. Kern, Electrical Transport Signature of the Magnetic Fluctuation-Structure Relation in $\alpha-\mathrm{RuCl}_{3}$ Nanoflakes, Nano Lett. 18, 3203 (2018).

[69] S. Mashhadi, Y. Kim, J. Kim, D. Weber, T. Taniguchi, K. Watanabe, N. Park, B. Lotsch, J. H. Smet, M. Burghard, and K. Kern, Spin-Split Band Hybridization in Graphene Proximitized with $\alpha-\mathrm{RuCl}_{3}$ Nanosheets, Nano Lett. 19, 4659 (2019).

[70] A. J. Willans, J. T. Chalker, and R. Moessner, Site Dilution in the Kitaev Honeycomb Model, Phys. Rev. B 84, 115146 (2011).

[71] K. Dhochak, R. Shankar, and V. Tripathi, Magnetic Impurities in the Honeycomb Kitaev Model, Phys. Rev. Lett. 105, 117201 (2010).

[72] M. Vojta, A. K. Mitchell, and F. Zschocke, Kondo Impurities in the Kitaev Spin Liquid: Numerical Renormalization Group Solution and Gauge-Flux-Driven Screening, Phys. Rev. Lett. 117, 037202 (2016).

[73] M. Barkeshli, E. Berg, and S. Kivelson, Coherent Transmutation of Electrons into Fractionalized Anyons, Science 346, 722 (2014).
[74] M. Barkeshli and C. Nayak, Superconductivity Induced Topological Phase Transition at the Edge of Even Denominator Fractional Quantum Hall States, arXiv:1507.06305.

[75] Reference [74] also briefly discusses applications to the non-Abelian spin liquid in Kitaev's honeycomb model, though their approach is very different from the one developed here.

[76] D. Aasen, E. Lake, and K. Walker, Fermion Condensation and Super Pivotal Categories, arXiv:1709.01941.

[77] A. Rahmani and M. Franz, Interacting Majorana Fermions, Rep. Prog. Phys. 82, 084501 (2019).

[78] Obtaining physical spin wave functions still requires enforcing the local constraint $D_{\mathbf{r}} \equiv b_{\mathbf{r}}^{x} b_{\mathbf{r}}^{y} b_{\mathbf{r}}^{z} c_{\mathbf{r}}=+1$ for all $\mathbf{r}$, which can be done by applying a projector $P=$ $\prod_{\mathbf{r}}\left[\left(1+D_{\mathbf{r}}\right) / 2\right]$ to many-body fermion states. Although $\left[\hat{u}_{\mathbf{r r}^{\prime}}, D_{\mathbf{r}^{\prime \prime}}\right] \neq 0$, gauge-invariant quantities (e.g., the energy) can nevertheless be exactly extracted from the free-fermion limit of Eq. (5) with fixed $\hat{u}_{\mathbf{r r}^{\prime}}$ values.

[79] E. H. Lieb, Flux Phase of the Half-Filled Band, Phys. Rev. Lett. 73, 2158 (1994).

[80] X.-Y. Song, Y.-. You, and L. Balents, Low-Energy Spin Dynamics of the Honeycomb Spin Liquid beyond the Kitaev Limit, Phys. Rev. Lett. 117, 037209 (2016).

[81] F. D. M. Haldane, Model for a Quantum Hall Effect without Landau Levels: Condensed-Matter Realization of the Parity Anomaly, Phys. Rev. Lett. 61, 2015 (1988).

[82] G. E. Volovik, The Gravitational Topological ChernSimons Term in a Film of Superfluid ${ }^{3} \mathrm{He}-A$, JETP Lett. 51, 125 (1990), https://www.researchgate.net/publication/ 239747601_The_gravitational-topological_Chern-Simons_ term_in_a_film_of_superfluid_3HA.

[83] C. L. Kane and M. P. A. Fisher, Quantized Thermal Transport in the Fractional Quantum Hall Effect, Phys. Rev. B 55, 15832 (1997).

[84] A. Cappelli, M. Huerta, and G. R. Zemba, Thermal Transport in Chiral Conformal Theories and Hierarchical Quantum Hall States, Nucl. Phys. B636, 568 (2002).

[85] In general, the edge velocity is expected to depend on details of the boundary and need not be spatially uniform, but for simplicity, we ignore such complications in this paper.

[86] J. C. Y. Teo and C. L. Kane, From Luttinger Liquid to NonAbelian Quantum Hall States, Phys. Rev. B 89, 085101 (2014).

[87] E. O'Brien and P. Fendley, Lattice Supersymmetry and Order-Disorder Coexistence in the Tricritical Ising Model, Phys. Rev. Lett. 120, 206403 (2018).

[88] N. Sannomiya and H. Katsura, Supersymmetry Breaking and Nambu-Goldstone Fermions in Interacting Majorana Chains, Phys. Rev. D 99, 045002 (2019).

[89] P. Fendley, Free Fermions in Disguise, J. Phys. A 52, 335002 (2019).

[90] In the purely 1D setting under consideration, $T$ symmetry is anomalous because it changes the sign of the total fermion-parity operator $P=\prod_{a}\left(i \gamma_{2 a} \gamma_{2 a+1}\right)$.

[91] At $t^{\prime}=0$, the chain also preserves a unitary reflection symmetry that sends $\gamma_{a} \rightarrow(-1)^{a} \gamma_{-a}$, but this symmetry does not play a role in our discussion.

[92] We caution, however, that physical fermions governed by the $1 \mathrm{D}$ lattice model do not realize Ising non-Abelian 
anyons in the same sense as the spin liquid. In particular, explicitly breaking the anomalous translation symmetry $T$ in the $1 \mathrm{D}$ model generically confines the domain walls, whereas in the spin-liquid interface, no symmetry is required for their deconfinement.

[93] A. Rahmani, X. Zhu, M. Franz, and I. Affleck, Emergent Supersymmetry from Strongly Interacting Majorana Zero Modes, Phys. Rev. Lett. 115, 166401 (2015).

[94] A. Rahmani, X. Zhu, M. Franz, and I. Affleck, Phase Diagram of the Interacting Majorana Chain Model, Phys. Rev. B 92, 235123 (2015).

[95] Spin-orbit coupling facilitates such processes. For instance, spin-orbit interactions in the superconductor generically yield a triplet component at the interface, enabling injection and removal of Cooper pairs from the quantum-Hall system even if the quantum-Hall edge state exhibits perfect spin polarization.

[96] O. Gamayun, J. A. Hutasoit, and V. V. Cheianov, TwoTerminal Transport along a Proximity-Induced Superconducting Quantum Hall Edge, Phys. Rev. B 96, 241104 (R) (2017).

[97] Y. Wan and C. Wang, Fermion Condensation and Gapped Domain Walls in Topological Orders, J. High Energy Phys. 03 (2017) 172.

[98] We stress the importance of a $\nu=1$ edge in the arguments presented here. For a $\nu=2$ quantum-Hall system, by contrast, Andreev processes do not freeze out at low energies. In this alternative setting, Fermi statistics allows a pairing term $\Delta\left(\psi_{R 1} \psi_{R 2}+\right.$ H.c. $)$, where $\psi_{R 1}$ and $\psi_{R 2}$ describe fermions in the two edge channels at the $\nu=2$ boundary. Such a pairing term does not vanish at zero momentum. As a corollary, at zero-incident-electron energy the momenta for the modes beneath the superconductor need not vanish-allowing a finite $\delta \phi$ even at asymptotically low energies.

[99] J. Wang, Q. Zhou, B. Lian, and S.-C. Zhang, Chiral Topological Superconductor and Half-Integer Conductance Plateau from Quantum Anomalous Hall Plateau Transition, Phys. Rev. B 92, 064520 (2015).

[100] B. Lian, J. Wang, and S.-C. Zhang, Edge-State-Induced Andreev Oscillation in Quantum Anomalous Hall Insulator-Superconductor Junctions, Phys. Rev. B 93, 161401(R) (2016).

[101] C.-Z. Chen, J. J. He, D.-H. Xu, and K. T. Law, Effects of Domain Walls in Quantum Anomalous Hall Insulator/ Superconductor Heterostructures, Phys. Rev. B 96, 041118(R) (2017).

[102] C.-Z. Chen, J. J. He, D.-H. Xu, and K. T. Law, Emergent Josephson Current of $n=1$ Chiral Topological Superconductor in Quantum Anomalous Hall Insulator/ Superconductor Heterostructures, Phys. Rev. B 98, 165439 (2018).

[103] C.-A. Li, J. Li, and S.-Q. Shen, Majorana-Josephson Interferometer, Phys. Rev. B 99, 100504(R) (2019).

[104] B. Lian and J. Wang, Distribution of Conductances in Chiral Topological Superconductor Junctions, Phys. Rev. B 99, 041404(R) (2019).

[105] Q. L. He, L. Pan, A. L. Stern, E. C. Burks, X. Che, G. Yin, J. Wang, B. Lian, Q. Zhou, E. S. Choi, K. Murata, X. Kou, Z. Chen, T. Nie, Q. Shao, Y. Fan, S.-C. Zhang, K. Liu,
J. Xia, and K. L. Wang, Chiral Majorana Fermion Modes in a Quantum Anomalous Hall Insulator-Superconductor Structure, Science 357, 294 (2017).

[106] W. Ji and X.-G. Wen, $\frac{1}{2}\left(e^{2} / h\right)$ Conductance Plateau without $1 D$ Chiral Majorana Fermions, Phys. Rev. Lett. 120, 107002 (2018).

[107] Y. Huang, F. Setiawan, and J. D. Sau, Disorder-Induced Half-Integer Quantized Conductance Plateau in Quantum Anomalous Hall Insulator-Superconductor Structures, Phys. Rev. B 97, 100501(R) (2018).

[108] M. Kayyalha, D. Xiao, R. Zhang, J. Shin, J. Jiang, F. Wang, Y.-F. Zhao, R. Xiao, L. Zhang, K. M. Fijalkowski, P. Mandal, M. Winnerlein, C. Gould, Q. Li, L. W. Molenkamp, M. H. W. Chan, N. Samarth, and C.-Z. Chang, Absence of Evidence for Chiral Majorana Modes in Quantum Anomalous Hall-Superconductor Devices, Science 367, 64 (2020).

[109] B. Lian, J. Wang, X.-Q. Sun, A. Vaezi, and S.-C. Zhang, Quantum Phase Transition of Chiral Majorana Fermions in the Presence of Disorder, Phys. Rev. B 97, 125408 (2018).

[110] A. F. Young, C. R. Dean, L. Wang, H. Ren, P. CaddenZimansky, K. Watanabe, T. Taniguchi, J. Hone, K. L. Shepard, and P. Kim, Spin and Valley Quantum Hall Ferromagnetism in Graphene, Nat. Phys. 8, 550 (2012).

[111] F. Amet, C. T. Ke, I. V. Borzenets, J. Wang, K. Watanabe, T. Taniguchi, R. S. Deacon, M. Yamamoto, Y. Bomze, S. Tarucha, and G. Finkelstein, Supercurrent in the Quantum Hall Regime, Science 352, 966 (2016).

[112] L. Zhao, E. G. Arnault, A. Bondarev, A. Seredinski, T. Larson, A. W. Draelos, H. Li, K. Watanabe, T. Taniguchi, F. Amet, H. U. Baranger, and G. Finkelstein, Interference of Chiral Andreev Edge States, arXiv:1907.01722.

[113] G.-H. Lee, K.-F. Huang, D. K. Efetov, D. S. Wei, S. Hart, T. Taniguchi, K. Watanabe, A. Yacoby, and P. Kim, Inducing Superconducting Correlation in Quantum Hall Edge States, Nat. Phys. 13, 693 (2017).

[114] M. Popinciuc, V. E. Calado, X. L. Liu, A. R. Akhmerov, T. M. Klapwijk, and L. M. K. Vandersypen, Zero-Bias Conductance Peak and Josephson Effect in GrapheneNbTiN Junctions, Phys. Rev. B 85, 205404 (2012).

[115] S. Cao, S. Shen, L. Chen, S. Yuan, B. Kang, and J. Zhang, Superconductivity at $14.6 \mathrm{~K}$ in $\mathrm{Fe}(\mathrm{SeTe})$ Single Crystal and the Role of Excess Fe, J. Appl. Phys. 110, 033914 (2011).

[116] P. Fendley, M. P. A. Fisher, and C. Nayak, Boundary Conformal Field Theory and Tunneling of Edge Quasiparticles in Non-Abelian Topological States, Ann. Phys. (Amsterdam) 324, 1547 (2009).

[117] C. L. Kane, Telegraph Noise and Fractional Statistics in the Quantum Hall Effect, Phys. Rev. Lett. 90, 226802 (2003).

[118] E. Grosfeld, S. H. Simon, and A. Stern, Switching Noise as a Probe of Statistics in the Fractional Quantum Hall Effect, Phys. Rev. Lett. 96, 226803 (2006).

[119] B. Rosenow and S. H. Simon, Telegraph Noise and the Fabry-Perot Quantum Hall Interferometer, Phys. Rev. B 85, 201302(R) (2012).

[120] S. An, P. Jiang, H. Choi, W. Kang, S. H. Simon, L. N. Pfeiffer, K. W. West, and K. W. Baldwin, Braiding of 
Abelian and Non-Abelian Anyons in the Fractional Quantum Hall Effect, arXiv:1112.3400.

[121] We stress that observing this conductance dichotomy does not require random events that displace Ising anyons by macroscopic distances. Rather, observing a jump to approximately $\left(2 e^{2} / h\right)$ conductance requires an Ising-anyon pair to be separated by distances of order the Majorana edge-state decay length such that one of the anyons couples negligibly to the gapless edge mode.

[122] P. Fendley, M. P. A. Fisher, and C. Nayak, Edge States and Tunneling of Non-Abelian Quasiparticles in the 5/2 Quantum Hall State and $p+i p$ Superconductors, Phys. Rev. B 75, 045317 (2007).

[123] W. Bishara and C. Nayak, Edge States and Interferometers in the Pfaffian and Anti-Pfaffian States of the $\nu=\frac{5}{2}$ Quantum Hall System, Phys. Rev. B 77, 165302 (2008).

[124] P. Bonderson, D. J. Clarke, C. Nayak, and K. Shtengel, Implementing Arbitrary Phase Gates with Ising Anyons, Phys. Rev. Lett. 104, 180505 (2010).

[125] J. Nilsson and A. R. Akhmerov, Theory of Non-Abelian Fabry-Perot Interferometry in Topological Insulators, Phys. Rev. B 81, 205110 (2010).

[126] For a discussion of energy partitioning in the Luttingerliquid context, see Ref. [127].

[127] T. Karzig, G. Refael, L. I. Glazman, and F. von Oppen, Energy Partitioning of Tunneling Currents into Luttinger Liquids, Phys. Rev. Lett. 107, 176403 (2011).

[128] One might have naively guessed that Ising-anyon tunneling instead admits a perturbative treatment provided the incoming fermion momentum $k_{e}$ is sufficiently large that $t_{\sigma} /\left(v_{e} k_{e}^{7 / 8}\right) \ll 1$. When this inequality holds, it would appear that one is probing the system at high energies for which $t_{\sigma}$ has not yet flowed to strong coupling. The fallacy in this argument stems from energy partitioning. Regardless of the magnitude of $k_{e}$, at the constriction the incident fermion splinters into Ising anyons that share the incident energy in all permissible ways. In particular, the allowed partitionings include cases where an Ising-anyon tunneling across the constriction carries arbitrarily small momentum, and for such events $t_{\sigma}$ can not be regarded as weak. Consequently, finite length $L_{a}$ is required to define a perturbative regime, corresponding to the quoted inequality $t_{\sigma} L_{a}^{7 / 8} / v_{e} \ll 1$.

[129] If the bulk Ising anyon's "partner" was instead dragged to the gapless part of the edge in the left interferometer half, paths (ii) and (iii) would acquire an additional $\pi$ phase.

[130] A. Milsted, L. Seabra, I. C. Fulga, C. W. J. Beenakker, and E. Cobanera, Statistical Translation Invariance Protects a Topological Insulator from Interactions, Phys. Rev. B 92, 085139 (2015).

[131] Actually, in the full microscopic model, $\delta \epsilon(k)$ reduces the overall bandwidth even though the velocity remains fixed. This effect likely yields a slightly smaller critical $U$ compared to what would occur if the bandwidth was also fixed.

[132] For a chiral CFT, one can transform between the "position slice" quantization and the "time slice" quantization via a Wick rotation (real time to imaginary time), a $90^{\circ}$ Euclidean rotation (exchange time and space), and then another Wick rotation (imaginary time back to real time).

[133] C. Nayak and F. Wilczek, 2n-Quasihole States Realize $2^{n-1}$-Dimensional Spinor Braiding Statistics in Paired Quantum Hall States, Nucl. Phys. B479, 529 (1996).

[134] Wolfram Research, Complete Elliptic Integral of the First Kind: Formula 08.02.04.0009, 2001 (accessed 2019), http://functions.wolfram.com/08.02.04.0009.01.

[135] Wolfram Research, Complete Elliptic Integral of the First Kind: Formula 08.02.17.0001, 2001 (accessed 2019), http://functions.wolfram.com/08.02.17.0001.01.

[136] Wolfram Research, Complete Elliptic Integral of the Second Kind: Formula 08.01.04.0009, 2001 (accessed 2019), http://functions.wolfram.com/08.01.04.0009.01.

[137] Wolfram Research, Complete Elliptic Integral of the Second Kind: Formula 08.01.17.0002, 2001 (accessed 2019), http://functions.wolfram.com/08.01.17.0002.01. 